Supplementary Information

for

\title{
Revealing Mechanism and Origin of Reactivity of Au(I)-Catalyzed Functionalized Indenone Formation of Cyclic and Acyclic Acetals of Alkynylaldehydes
}

Aqeel A. Hussein ${ }^{1 *}$ and Hafiz S. Ali ${ }^{2 \ddagger}$

${ }^{1}$ College of Dentistry, University of Al-Ameed, Karbala PO Box 198, Iraq.

2 Department of Chemistry and Manchester Institute of Biotechnology, The University of Manchester, 131 Princess Street, Manchester M1 7DN, UK.

*Email: aqeel_alaa85@yahoo.com 


\begin{tabular}{|c|c|c|c|}
\hline Structure & $\begin{array}{l}\text { Thermal Gibbs free } \\
\text { energy correction } \\
(353.15 \mathrm{~K})\end{array}$ & Total Energy (SMD, DCE) & $\begin{array}{l}\text { Gibbs free energy at } \\
353.15 \mathrm{~K} \text { (SMD, DCE) }\end{array}$ \\
\hline 1a & 0.235373 & -921.541393214 & -921.30602021 \\
\hline IPrAu & 0.486377 & -1296.263800220 & -1295.77742322 \\
\hline Int1a & 0.748789 & -2217.855601280 & -2217.10681228 \\
\hline TS1a & 0.750435 & -2217.829406560 & -2217.07897156 \\
\hline Int2a & 0.753056 & -2217.864802300 & -2217.11174630 \\
\hline TS2a & 0.751770 & -2217.836672650 & -2217.08490265 \\
\hline Int3a & 0.754389 & -2217.867740920 & -2217.11335192 \\
\hline TS3a & 0.751760 & -2217.838925090 & -2217.08716509 \\
\hline Int4a & 0.757169 & -2217.897332470 & -2217.14016347 \\
\hline $2 a$ & 0.241683 & -921.580611405 & -921.33892841 \\
\hline TS1' & 0.234630 & -921.467909399 & -921.23327940 \\
\hline Int1' & 0.234658 & -921.517202481 & -921.28254448 \\
\hline TS2' & 0.237366 & -921.470659490 & -921.23329349 \\
\hline int2' & 0.237918 & -921.482766874 & -921.24484887 \\
\hline TS3' & 0.235941 & -921.466544309 & -921.23060331 \\
\hline TS1a' & 0.748219 & -2217.812659320 & -2217.06444032 \\
\hline Int2a' & 0.753268 & -2217.863221640 & -2217.10995364 \\
\hline TS2a & 0.757520 & -2217.854311870 & -2217.09679187 \\
\hline Int3a' & 0.757287 & -2217.899109340 & -2217.14182234 \\
\hline Int1b & 0.720330 & -2103.277654120 & -2102.55732412 \\
\hline TS1b & 0.721935 & -2103.249706330 & -2102.52777133 \\
\hline Int1c & 0.754996 & -2331.259007750 & -2330.50401175 \\
\hline TS1c & 0.756059 & -2331.231729990 & -2330.47567099 \\
\hline Int1d & 0.745241 & -2422.449012450 & -2421.70377145 \\
\hline TS1d & 0.743929 & -2422.422141620 & -2421.67821262 \\
\hline Int1e & 0.776950 & -2332.433968550 & -2331.65701855 \\
\hline TS1e & 0.778658 & -2332.405810710 & -2331.62715271 \\
\hline 1f & 0.132196 & -575.801779210 & -575.668819210 \\
\hline Int1f & 0.649591 & -1872.110893900 & -1871.46130290 \\
\hline TS1f & 0.645767 & -1872.079370420 & -1871.43360342 \\
\hline Int2f & 0.650682 & -1872.132764320 & -1871.48208232 \\
\hline TS2f & 0.652839 & -1872.110556610 & -1871.45771761 \\
\hline Int3f & 0.652249 & -1872.135166490 & -1871.48291749 \\
\hline
\end{tabular}




\begin{tabular}{|l|l|l|l|}
\hline TS3f & 0.649697 & -1872.094519720 & -1871.44482272 \\
\hline Int4f & 0.653198 & -1872.160056850 & -1871.50685885 \\
\hline & & & \\
\hline TS1f' $^{\prime}$ & 0.645554 & -1872.077563200 & -1871.43200920 \\
\hline Int2' $^{\prime}$ & 0.649818 & -1872.126329710 & -1871.47651171 \\
\hline TS2f' & 0.654271 & -1872.100476400 & -1871.44620540 \\
\hline Int3f' $^{\prime}$ & 0.654329 & -1872.160087840 & -1871.50575884 \\
\hline & & & \\
\hline Int1g & 0.770087 & -2219.063069310 & -2218.29298231 \\
\hline TS1g & 0.767113 & -2219.024656440 & -2218.25754344 \\
\hline Int2g & 0.774774 & -2219.070234470 & -2218.29546047 \\
\hline TS2g & 0.775445 & -2219.057613430 & -2218.28216843 \\
\hline Int3g & 0.775695 & -2219.069888570 & -2218.29419357 \\
\hline TS3g & 0.771847 & -2219.035077060 & -2218.26323006 \\
\hline Int4g & 0.775517 & -2219.100097920 & -2218.32458092 \\
\hline & & & \\
\hline TS1g' & 0.773208 & -2219.038417660 & -2218.26520966 \\
\hline Int2g' & 0.772146 & -2219.055211760 & -2218.28306576 \\
\hline TS2g' & 0.775129 & -2219.044585320 & -2218.26945632 \\
\hline Int3g' $^{\prime}$ & 0.776384 & -2219.060264520 & -2218.28388052 \\
\hline TS3g' & 0.776860 & -2219.035227620 & -2218.25836762 \\
\hline Int4g' & 0.773115 & -2219.097485900 & -2218.32437090 \\
\hline
\end{tabular}




$\begin{array}{crrr}\text { 1a } & & & \\ \mathrm{C} & -3.50463300 & -0.16436800 & -0.38389800 \\ \mathrm{C} & -4.57464600 & -0.48265600 & 0.49038300 \\ \mathrm{C} & -5.27554700 & 0.54824400 & 1.11524800 \\ \mathrm{C} & -4.93289500 & 1.88541000 & 0.90027200 \\ \mathrm{C} & -3.87880600 & 2.20324700 & 0.04236600 \\ \mathrm{C} & -3.17229400 & 1.18772600 & -0.59647800 \\ \mathrm{C} & -2.76763700 & -1.18932300 & -1.04388600 \\ \mathrm{C} & -2.13844000 & -2.07372300 & -1.59373300 \\ \mathrm{H} & -6.10881400 & 0.28980700 & 1.75913700 \\ \mathrm{H} & -5.49209300 & 2.67410200 & 1.39635200 \\ \mathrm{H} & -3.60853100 & 3.24102800 & -0.13346600 \\ \mathrm{H} & -2.35232200 & 1.42476200 & -1.26750500 \\ \mathrm{C} & -1.41845000 & -3.14636100 & -2.18984600 \\ \mathrm{C} & -0.57816200 & -2.94346400 & -3.29794200 \\ \mathrm{C} & -1.53366300 & -4.45562400 & -1.66919000 \\ \mathrm{C} & 0.12527100 & -3.99963800 & -3.87546900 \\ \mathrm{H} & -0.47751400 & -1.94412500 & -3.71034800 \\ \mathrm{C} & -0.83791100 & -5.50914100 & -2.23832600 \\ \mathrm{H} & -2.17436500 & -4.62295500 & -0.80881900 \\ \mathrm{C} & -0.00242400 & -5.29109000 & -3.34686800 \\ \mathrm{H} & 0.76442800 & -3.80618400 & -4.72932800 \\ \mathrm{H} & -0.92036000 & -6.51692800 & -1.84350900 \\ \mathrm{O} & 0.63611700 & -6.39370500 & -3.83029200 \\ \mathrm{C} & 1.49573400 & -6.23975200 & -4.94825900 \\ \mathrm{H} & 2.32668500 & -5.55672700 & -4.72744900 \\ \mathrm{H} & 1.89314200 & -7.23417100 & -5.15935300 \\ \mathrm{H} & 0.95155800 & -5.87256100 & -5.82846300 \\ \mathrm{O} & -6.15727700 & -2.05542500 & 1.44492900 \\ \mathrm{O} & -3.94249200 & -2.64043400 & 1.46740600 \\ \mathrm{C} & -5.90460100 & -2.80041600 & 2.63706900 \\ \mathrm{H} & -6.49793100 & -2.37170800 & 3.45045100 \\ \mathrm{H} & -6.18780700 & -3.85310600 & 2.49715000 \\ \mathrm{C} & -4.39625200 & -2.64775500 & 2.81655200 \\ \mathrm{H} & -3.91575300 & -3.48228700 & 3.33297600 \\ \mathrm{H} & -4.14460500 & -1.70664400 & 3.32781800 \\ \mathrm{C} & -4.93753100 & -1.94367100 & 0.71935600 \\ \mathrm{H} & -5.02038400 & -2.46822100 & -0.24000300\end{array}$

$\begin{array}{lccc}\text { IPrAu } & & & \\ \mathrm{C} & 1.76657600 & 0.73014000 & 0.52627600 \\ \mathrm{C} & 3.36831600 & 2.20702300 & 1.00588100 \\ \mathrm{C} & 3.78489700 & 0.98728500 & 1.44098600 \\ \mathrm{H} & 3.83823500 & 3.17720300 & 1.04225800 \\ \mathrm{H} & 4.69234700 & 0.67733000 & 1.93485600 \\ \mathrm{~N} & 2.78066900 & 0.07914300 & 1.13704100 \\ \mathrm{~N} & 2.11326600 & 2.03300200 & 0.44017000 \\ \mathrm{Au} & 0.07815300 & -0.07728300 & -0.12785300 \\ \mathrm{C} & 1.31294100 & 3.09412100 & -0.14946100 \\ \mathrm{C} & 1.47346300 & 3.36416400 & -1.52368600 \\ \mathrm{C} & 0.43471100 & 3.81110100 & 0.68823000 \\ \mathrm{C} & 0.69267600 & 4.39539400 & -2.06014600 \\ \mathrm{C} & -0.31630700 & 4.83116900 & 0.09113400 \\ \mathrm{C} & -0.19228900 & 5.11954000 & -1.26547000 \\ \mathrm{H} & 0.78288800 & 4.63599300 & -3.11489800 \\ \mathrm{H} & -1.00532100 & 5.40867100 & 0.69954300 \\ \mathrm{H} & -0.78517900 & 5.91631800 & -1.70502600 \\ \mathrm{C} & 2.83122700 & -1.34330500 & 1.43398900 \\ \mathrm{C} & 3.40184200 & -2.20345400 & 0.47374700 \\ \mathrm{C} & 2.33249000 & -1.78336000 & 2.67691600 \\ \mathrm{C} & 3.44926000 & -3.56557200 & 0.79528000 \\ \mathrm{C} & 2.40882600 & -3.15718700 & 2.93717800 \\ \mathrm{C} & 2.95828700 & -4.03834400 & 2.00950500\end{array}$

$\begin{array}{rrr}3.88117500 & -4.26439700 & 0.08560100 \\ 2.03681400 & -3.54034400 & 3.88222700 \\ 3.00868900 & -5.09930900 & 2.23625500 \\ 0.28478300 & 3.53387800 & 2.18156200 \\ 0.93522800 & 2.69275800 & 2.44571300 \\ 0.74229900 & 4.74266700 & 3.02324200 \\ 0.67562400 & 4.50769500 & 4.09114000 \\ 1.77838400 & 5.01986000 & 2.79954500 \\ 0.11547400 & 5.62130900 & 2.83625600 \\ -1.15691800 & 3.12291100 & 2.54041900 \\ -1.86977400 & 3.92835500 & 2.33352600 \\ -1.47607500 & 2.24149000 & 1.97130900 \\ -1.22816900 & 2.88364600 & 3.60711300 \\ 1.74055400 & -0.84440300 & 3.72417100 \\ 2.58051400 & -0.84638900 & 5.01775700 \\ 3.62319400 & -0.57389900 & 4.82063200 \\ 2.17102800 & -0.12801400 & 5.73632300 \\ 2.57896700 & -1.83193900 & 5.49561900 \\ 3.96734000 & -1.71859700 & -0.85830900 \\ 3.80484500 & -0.63758800 & -0.93220900 \\ 2.44102300 & 2.59975100 & -2.42301300 \\ 3.51248700 & 3.53371700 & -3.02147300 \\ 3.06716000 & 4.28831300 & -3.67864200 \\ 4.06897300 & 4.06077000 & -2.23871300 \\ 4.22761000 & 2.95590400 & -3.61701000 \\ 3.24357600 & -2.36884900 & -2.05385300 \\ 2.16415100 & -2.17848100 & -2.02155800 \\ 3.63235800 & -1.96661000 & -2.99574900 \\ 3.38954600 & -3.45419500 & -2.07264800 \\ 5.48947100 & -1.95496000 & -0.93787300 \\ 5.88600600 & -1.55851400 & -1.87900600 \\ 6.01706200 & -1.46387200 & -0.11270700 \\ 5.73158500 & -3.02257100 & -0.89810600 \\ 0.26630700 & -1.18365600 & 4.02080200 \\ -0.34461500 & -1.14772200 & 3.11064800 \\ 0.16180700 & -2.18505900 & 4.45210300 \\ -0.15003500 & -0.46860800 & 4.73880100 \\ 1.76363700 & 0.17633000 & 3.32709800 \\ 1.69245900 & 1.83384400 & -3.53186800 \\ 2.39988600 & 1.26304000 & -4.14343200 \\ 0.96237800 & 1.13189000 & -3.11043400 \\ 1.15239600 & 2.51588200 & -4.19756700 \\ 2.96741200 & 1.85595000 & -1.81476500\end{array}$

$\begin{array}{crrr}\text { Int1a } & & & \\ \mathrm{C} & -3.20615400 & 0.21182700 & -0.24063500 \\ \mathrm{C} & -4.34902900 & -0.39390600 & 0.33450600 \\ \mathrm{C} & -5.39567400 & 0.42063100 & 0.76558600 \\ \mathrm{C} & -5.32610900 & 1.81013200 & 0.63735900 \\ \mathrm{C} & -4.19700700 & 2.40516700 & 0.07498000 \\ \mathrm{C} & -3.13685300 & 1.60991700 & -0.35501100 \\ \mathrm{C} & -2.11421300 & -0.61646000 & -0.71096600 \\ \mathrm{C} & -1.59809700 & -1.60727000 & -1.26044400 \\ \mathrm{H} & -6.27392200 & -0.05054800 & 1.19091700 \\ \mathrm{H} & -6.15598700 & 2.42387200 & 0.97541700 \\ \mathrm{H} & -4.13809100 & 3.48428600 & -0.03032600 \\ \mathrm{H} & -2.25119100 & 2.06469000 & -0.78855600 \\ \mathrm{C} & 1.73932800 & 0.70553100 & 0.48362100 \\ \mathrm{C} & 3.36320700 & 2.19014700 & 0.97524600 \\ \mathrm{C} & 3.77793700 & 0.97092800 & 1.40743000 \\ \mathrm{H} & 3.83361400 & 3.15981500 & 1.01539700 \\ \mathrm{H} & 4.68505400 & 0.65979600 & 1.90087600 \\ \mathrm{~N} & 2.76869000 & 0.06890300 & 1.09871200\end{array}$




\begin{tabular}{|c|c|c|c|}
\hline $\mathrm{N}$ & 2.10773200 & 2.01067900 & 0.41098900 \\
\hline $\mathrm{Au}$ & 0.00174100 & -0.09664300 & -0.18305600 \\
\hline $\mathrm{C}$ & 1.30071200 & 3.07964900 & -0.14424000 \\
\hline $\mathrm{C}$ & 1.38160500 & 3.33678400 & -1.52757900 \\
\hline $\mathrm{C}$ & 0.48733200 & 3.82568500 & 0.73284400 \\
\hline $\mathrm{C}$ & 0.59354000 & 4.38020700 & -2.02956800 \\
\hline $\mathrm{C}$ & -0.27490200 & 4.85863700 & 0.17238900 \\
\hline $\mathrm{C}$ & -0.22609000 & 5.13276700 & -1.19221000 \\
\hline $\mathrm{H}$ & 0.62850100 & 4.61005000 & -3.09023700 \\
\hline $\mathrm{H}$ & -0.91155200 & 5.45796200 & 0.81619400 \\
\hline $\mathrm{H}$ & -0.82373300 & 5.94107300 & -1.60413700 \\
\hline $\mathrm{C}$ & 2.82469600 & -1.34726400 & 1.40474200 \\
\hline $\mathrm{C}$ & 3.46971000 & -2.20417200 & 0.49044900 \\
\hline $\mathrm{C}$ & 2.25971800 & -1.79541200 & 2.61564000 \\
\hline $\mathrm{C}$ & 3.53744800 & -3.56161200 & 0.82731700 \\
\hline $\mathrm{C}$ & 2.35545000 & -3.16450600 & 2.89482300 \\
\hline $\mathrm{C}$ & 2.98688300 & -4.03837300 & 2.01397600 \\
\hline $\mathrm{H}$ & 4.03090500 & -4.25374600 & 0.15169500 \\
\hline $\mathrm{H}$ & 1.93515500 & -3.54864600 & 3.81928000 \\
\hline $\mathrm{H}$ & 3.05454900 & -5.09538500 & 2.25555000 \\
\hline $\mathrm{C}$ & 0.42260700 & 3.56551400 & 2.23564700 \\
\hline $\mathrm{H}$ & 1.04271100 & 2.69188100 & 2.46126900 \\
\hline $\mathrm{C}$ & 1.00125700 & 4.75357100 & 3.03098000 \\
\hline $\mathrm{H}$ & 0.99595700 & 4.53137000 & 4.10392800 \\
\hline $\mathrm{H}$ & 2.03322000 & 4.97259800 & 2.73466500 \\
\hline $\mathrm{H}$ & 0.41125100 & 5.66389300 & 2.87649400 \\
\hline $\mathrm{C}$ & -1.00922600 & 3.23407900 & 2.69890200 \\
\hline $\mathrm{H}$ & -1.68929200 & 4.07940900 & 2.54512900 \\
\hline $\mathrm{H}$ & -1.41706500 & 2.37410800 & 2.15710300 \\
\hline $\mathrm{H}$ & -1.01319300 & 2.99920400 & 3.76925900 \\
\hline $\mathrm{C}$ & 1.58070800 & -0.86441400 & 3.61593100 \\
\hline $\mathrm{C}$ & 2.36134900 & -0.80907800 & 4.94486100 \\
\hline $\mathrm{H}$ & 3.39775200 & -0.48953100 & 4.78883200 \\
\hline $\mathrm{H}$ & 1.88767700 & -0.10170500 & 5.63476700 \\
\hline $\mathrm{H}$ & 2.38588900 & -1.78791800 & 5.43659000 \\
\hline $\mathrm{C}$ & 4.09659200 & -1.71342800 & -0.81148900 \\
\hline $\mathrm{H}$ & 3.86477300 & -0.64943900 & -0.92615900 \\
\hline $\mathrm{C}$ & 2.28620900 & 2.54934800 & -2.47076500 \\
\hline $\mathrm{C}$ & 3.37446400 & 3.45220800 & -3.08602200 \\
\hline $\mathrm{H}$ & 2.93767200 & 4.23878800 & -3.71126300 \\
\hline $\mathrm{H}$ & 3.97691900 & 3.93925600 & -2.31135000 \\
\hline $\mathrm{H}$ & 4.04673100 & 2.86048900 & -3.71744900 \\
\hline $\mathrm{C}$ & 3.50630600 & -2.43554000 & -2.03789000 \\
\hline $\mathrm{H}$ & 2.41717400 & -2.32671900 & -2.07881300 \\
\hline $\mathrm{H}$ & 3.92908700 & -2.01845200 & -2.95914800 \\
\hline $\mathrm{H}$ & 3.73571900 & -3.50682800 & -2.02549000 \\
\hline $\mathrm{C}$ & 5.63245400 & -1.84821200 & -0.77529200 \\
\hline $\mathrm{H}$ & 6.07242400 & -1.45237900 & -1.69756700 \\
\hline $\mathrm{H}$ & 6.06510300 & -1.30025700 & 0.06925200 \\
\hline $\mathrm{H}$ & 5.93860100 & -2.89627200 & -0.68182900 \\
\hline $\mathrm{C}$ & 0.10898400 & -1.25727700 & 3.85168700 \\
\hline $\mathrm{H}$ & -0.45917300 & -1.26352500 & 2.91456200 \\
\hline $\mathrm{H}$ & 0.02572400 & -2.25232500 & 4.30325900 \\
\hline $\mathrm{H}$ & -0.36588300 & -0.54376400 & 4.53480000 \\
\hline $\mathrm{H}$ & 1.57864700 & 0.14750500 & 3.19828000 \\
\hline $\mathrm{C}$ & 1.47503200 & 1.83251400 & -3.56788200 \\
\hline $\mathrm{H}$ & 2.14189400 & 1.23945400 & -4.20412400 \\
\hline $\mathrm{H}$ & 0.72749000 & 1.16001700 & -3.13121300 \\
\hline $\mathrm{H}$ & 0.94957800 & 2.54579700 & -4.21274700 \\
\hline $\mathrm{H}$ & 2.79723400 & 1.77428100 & -1.89047300 \\
\hline $\mathrm{C}$ & -1.10460800 & -2.80449800 & -1.84063100 \\
\hline $\mathrm{C}$ & -0.57969000 & -2.83131600 & -3.14772500 \\
\hline $\mathrm{C}$ & -1.19092200 & -4.01471200 & -1.10806400 \\
\hline $\mathrm{C}$ & -0.14589700 & -4.02299900 & -3.71531900 \\
\hline $\mathrm{H}$ & -0.51852000 & -1.90956200 & -3.71813100 \\
\hline $\mathrm{C}$ & -0.76710000 & -5.20139500 & -1.67301500 \\
\hline $\mathrm{H}$ & -1.61516600 & -3.99477800 & -0.10890500 \\
\hline
\end{tabular}

$\begin{array}{lrrr}\mathrm{C} & -0.23766700 & -5.21897100 & -2.97997400 \\ \mathrm{H} & 0.25057000 & -4.01887800 & -4.72347600 \\ \mathrm{H} & -0.83428100 & -6.13978700 & -1.13260300 \\ \mathrm{O} & 0.14817300 & -6.42737400 & -3.43309900 \\ \mathrm{C} & 0.66941200 & -6.54386900 & -4.75702100 \\ \mathrm{H} & 1.58713100 & -5.95552300 & -4.87446600 \\ \mathrm{H} & 0.89523600 & -7.60188500 & -4.89098200 \\ \mathrm{H} & -0.07049200 & -6.22993100 & -5.50240500 \\ \mathrm{O} & -5.69693300 & -2.29033700 & 0.95660400 \\ \mathrm{O} & -3.44172000 & -2.43605400 & 1.35594600 \\ \mathrm{C} & -5.48805700 & -3.32036000 & 1.92472900 \\ \mathrm{H} & -6.31433700 & -3.28778300 & 2.63823400 \\ \mathrm{H} & -5.45791400 & -4.30701000 & 1.44174800 \\ \mathrm{C} & -4.13245400 & -2.93694200 & 2.50575000 \\ \mathrm{H} & -3.56136200 & -3.77773000 & 2.90697200 \\ \mathrm{H} & -4.22870300 & -2.15875000 & 3.27493600 \\ \mathrm{C} & -4.43428200 & -1.90891100 & 0.45976300 \\ \mathrm{H} & -4.24929600 & -2.38081800 & -0.51665700\end{array}$

$\begin{array}{lrrr}\text { TS1a } & & & \\ \mathrm{C} & -3.27009000 & 0.06411000 & -0.11396200 \\ \mathrm{C} & -4.59876000 & -0.38488200 & -0.28437400 \\ \mathrm{C} & -5.67453600 & 0.49939300 & -0.18831100 \\ \mathrm{C} & -5.44793500 & 1.83623000 & 0.13541200 \\ \mathrm{C} & -4.14067600 & 2.28882600 & 0.33314400 \\ \mathrm{C} & -3.06276400 & 1.41463800 & 0.20518300 \\ \mathrm{C} & -2.15739000 & -0.87735600 & -0.34201500 \\ \mathrm{C} & -2.35724000 & -2.06588200 & -0.86339600 \\ \mathrm{H} & -6.68295500 & 0.13252900 & -0.35229900 \\ \mathrm{H} & -6.28626000 & 2.51912000 & 0.23432500 \\ \mathrm{H} & -3.95830300 & 3.33169100 & 0.57549000 \\ \mathrm{H} & -2.04846800 & 1.77909600 & 0.33316300 \\ \mathrm{C} & 1.76670500 & 0.14761500 & 0.53902800 \\ \mathrm{C} & 3.60730400 & 1.41299600 & 0.90201500 \\ \mathrm{C} & 3.95658000 & 0.11722200 & 1.10605900 \\ \mathrm{H} & 4.17927700 & 2.32503300 & 0.96762600 \\ \mathrm{H} & 4.89535300 & -0.33300400 & 1.38754500 \\ \mathrm{~N} & 2.81715100 & -0.64590400 & 0.88081000 \\ \mathrm{~N} & 2.26206800 & 1.41476500 & 0.55686600 \\ \mathrm{Au} & -0.15967000 & -0.39647900 & 0.07888100 \\ \mathrm{C} & 1.49653300 & 2.61213000 & 0.27666100 \\ \mathrm{C} & 1.42279100 & 3.06892000 & -1.05420900 \\ \mathrm{C} & 0.87557200 & 3.27716900 & 1.35358400 \\ \mathrm{C} & 0.67881400 & 4.23161300 & -1.29169500 \\ \mathrm{C} & 0.14676700 & 4.43557800 & 1.05469400 \\ \mathrm{C} & 0.04632800 & 4.90775700 & -0.25182700 \\ \mathrm{H} & 0.59803100 & 4.61354400 & -2.30490200 \\ \mathrm{H} & -0.34232600 & 4.97854600 & 1.85808400 \\ \mathrm{H} & -0.52102100 & 5.81081900 & -0.45925600 \\ \mathrm{C} & 2.77858900 & -2.08691500 & 1.02011800 \\ \mathrm{C} & 3.09469900 & -2.88038500 & -0.10051600 \\ \mathrm{C} & 2.46800400 & -2.63012300 & 2.28322200 \\ \mathrm{C} & 3.08905500 & -4.26961000 & 0.07650200 \\ \mathrm{C} & 2.47708700 & -4.02609200 & 2.39937100 \\ \mathrm{C} & 2.78624700 & -4.83806200 & 1.31102100 \\ \mathrm{H} & 3.33169800 & -4.91380100 & -0.76338700 \\ \mathrm{H} & 2.24888800 & -4.48135900 & 3.35872500 \\ \mathrm{H} & 2.79929400 & -5.91840500 & 1.42741500 \\ \mathrm{H} & -0.40254600 & 2.46364700 & 3.39153600 \\ \mathrm{C} & 1.56643800 & 1.87463500 & 2.81336100 \\ \mathrm{H} & 1.72534200 & 3.82536000 & 3.67581000 \\ \mathrm{C} & 1.83865900 & 3.44234300 & 4.69624200 \\ \mathrm{H} & 2.72446400 & 4.04035300 & 3.28074600 \\ \mathrm{H} & & & \\ \mathrm{H} & & & \end{array}$




\begin{tabular}{|c|c|c|c|}
\hline $\mathrm{H}$ & -1.04542200 & 3.34973900 & 3.44472100 \\
\hline $\mathrm{H}$ & -0.91950800 & 1.70586900 & 2.79233300 \\
\hline $\mathrm{H}$ & -0.29270700 & 2.07537900 & 4.41051700 \\
\hline $\mathrm{C}$ & 2.14332000 & -1.77133500 & 3.50216900 \\
\hline $\mathrm{C}$ & 3.17644600 & -1.97830200 & 4.62782200 \\
\hline $\mathrm{H}$ & 4.19428700 & -1.76498900 & 4.28283000 \\
\hline $\mathrm{H}$ & 2.95750400 & -1.31360100 & 5.47103800 \\
\hline $\mathrm{H}$ & 3.16149700 & -3.00730700 & 5.00397200 \\
\hline $\mathrm{C}$ & 3.44534200 & -2.29064500 & -1.46349900 \\
\hline $\mathrm{H}$ & 3.38216800 & -1.20038100 & -1.39119000 \\
\hline $\mathrm{C}$ & 2.11502900 & 2.36131900 & -2.21500400 \\
\hline $\mathrm{C}$ & 3.16872200 & 3.26989400 & -2.87930400 \\
\hline $\mathrm{H}$ & 2.70836900 & 4.15283900 & -3.33673800 \\
\hline $\mathrm{H}$ & 3.91173100 & 3.61993500 & -2.15406500 \\
\hline $\mathrm{H}$ & 3.69641000 & 2.72430300 & -3.66975500 \\
\hline $\mathrm{C}$ & 2.43935900 & -2.73075900 & -2.54497000 \\
\hline $\mathrm{H}$ & 1.41696000 & -2.44375900 & -2.27574600 \\
\hline $\mathrm{H}$ & 2.68603900 & -2.26130400 & -3.50417300 \\
\hline $\mathrm{H}$ & 2.45716800 & -3.81671200 & -2.69249100 \\
\hline $\mathrm{C}$ & 4.89027700 & -2.63932300 & -1.87254900 \\
\hline $\mathrm{H}$ & 5.13880600 & -2.16555000 & -2.82885300 \\
\hline $\mathrm{H}$ & 5.61359600 & -2.29514400 & -1.12494800 \\
\hline $\mathrm{H}$ & 5.02477100 & -3.72030300 & -1.99202300 \\
\hline $\mathrm{C}$ & 0.71044900 & -2.02774800 & 4.00936100 \\
\hline $\mathrm{H}$ & -0.02893600 & -1.82531100 & 3.22608300 \\
\hline $\mathrm{H}$ & 0.57976700 & -3.06405500 & 4.34157000 \\
\hline $\mathrm{H}$ & 0.48798300 & -1.37663800 & 4.86238900 \\
\hline $\mathrm{H}$ & 2.19410700 & -0.71927600 & 3.20484300 \\
\hline $\mathrm{C}$ & 1.09518100 & 1.84387300 & -3.24872800 \\
\hline $\mathrm{H}$ & 1.60949200 & 1.29849400 & -4.04835200 \\
\hline $\mathrm{H}$ & 0.36932900 & 1.16695200 & -2.78398600 \\
\hline $\mathrm{H}$ & 0.54013500 & 2.66730500 & -3.71242100 \\
\hline $\mathrm{H}$ & 2.64379000 & 1.48874900 & -1.81809700 \\
\hline $\mathrm{C}$ & -1.87756400 & -3.40538600 & -1.04174100 \\
\hline $\mathrm{C}$ & -2.02540700 & -4.06641100 & -2.27867700 \\
\hline $\mathrm{C}$ & -1.26262600 & -4.10522400 & 0.02639700 \\
\hline $\mathrm{C}$ & -1.57476900 & -5.36764100 & -2.46110800 \\
\hline $\mathrm{H}$ & -2.49775200 & -3.54348900 & -3.10566500 \\
\hline $\mathrm{C}$ & -0.79763500 & -5.39381200 & -0.15120900 \\
\hline $\mathrm{H}$ & -1.14474300 & -3.60964500 & 0.98430200 \\
\hline $\mathrm{C}$ & -0.95043300 & -6.04063700 & -1.39439600 \\
\hline $\mathrm{H}$ & -1.69914300 & -5.84634200 & -3.42505700 \\
\hline $\mathrm{H}$ & -0.30891900 & -5.93148400 & 0.65396700 \\
\hline $\mathrm{O}$ & -0.46796500 & -7.29740400 & -1.45948300 \\
\hline $\mathrm{C}$ & -0.58846000 & -8.02931600 & -2.67937700 \\
\hline $\mathrm{H}$ & -0.05143800 & -7.53124500 & -3.49514400 \\
\hline $\mathrm{H}$ & -0.13523000 & -9.00141200 & -2.48433400 \\
\hline $\mathrm{H}$ & -1.64019700 & -8.16513700 & -2.95724200 \\
\hline $\mathrm{O}$ & -5.80677400 & -2.07210300 & -1.55237300 \\
\hline $\mathrm{O}$ & -4.97573600 & -2.67258900 & 0.44838800 \\
\hline $\mathrm{C}$ & -6.48965400 & -3.29425800 & -1.18818500 \\
\hline $\mathrm{H}$ & -7.49780600 & -3.03243900 & -0.85289000 \\
\hline $\mathrm{H}$ & -6.54931500 & -3.93856300 & -2.06750400 \\
\hline $\mathrm{C}$ & -5.61547500 & -3.85671900 & -0.06378300 \\
\hline $\mathrm{H}$ & -4.85682700 & -4.55553000 & -0.43449800 \\
\hline $\mathrm{H}$ & -6.17371000 & -4.31317100 & 0.75426400 \\
\hline $\mathrm{C}$ & -4.83406600 & -1.81565600 & -0.61850400 \\
\hline $\mathrm{H}$ & -3.72399500 & -2.10441200 & -1.16791500 \\
\hline
\end{tabular}
Imaginary Frequency $=-639.6084$

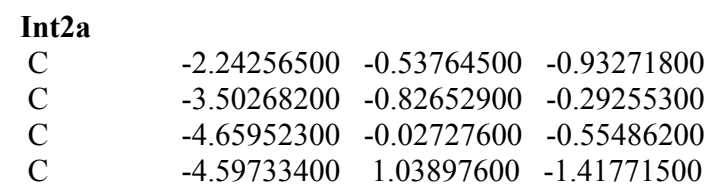

$\begin{array}{lll}-3.36680400 & 1.34522300 & -2.03829600\end{array}$ $\begin{array}{lll}-2.23355400 & 0.59417400 & -1.78597600\end{array}$ $\begin{array}{lll}-0.99121200 & -1.26876700 & -0.73622000\end{array}$ $\begin{array}{lll}-0.78432100 & -2.56514800 & -1.07333600\end{array}$ $\begin{array}{lll}-5.58877200 & -0.26824000 & -0.05128400\end{array}$ $\begin{array}{lll}-5.47769700 & 1.64301300 & -1.61027900\end{array}$ $\begin{array}{lll}-3.30597100 & 2.18664900 & -2.72310000\end{array}$ $\begin{array}{lll}-1.29290400 & 0.85595100 & -2.25871600\end{array}$ $\begin{array}{lll}2.18391700 & 0.92673200 & 0.78267300\end{array}$ $\begin{array}{lll}3.59373100 & 2.62087800 & 1.31249100\end{array}$ $\begin{array}{lll}4.17181200 & 1.46569600 & 1.72878900\end{array}$ $\begin{array}{llll}3.92534300 & 3.64564800 & 1.36915500\end{array}$ $\begin{array}{lll}5.11148400 & 1.27554900 & 2.22296800\end{array}$ $\begin{array}{lll}3.29662300 & 0.43805100 & 1.39662900\end{array}$ $\begin{array}{lll}2.37810400 & 2.27377200 & 0.73453100\end{array}$ $\begin{array}{lll}0.57854200 & -0.14440000 & 0.04825400\end{array}$ $\begin{array}{lll}1.45797600 & 3.22774000 & 0.15257700\end{array}$ $\begin{array}{lll}1.60712800 & 3.56329200 & -1.20809500\end{array}$ $\begin{array}{lll}0.46861400 & 3.79801500 & 0.97786500\end{array}$ $\begin{array}{llll}0.71033800 & 4.49897400 & -1.73956500\end{array}$ $\begin{array}{lll}-0.39899900 & 4.72827900 & 0.39192700\end{array}$ $\begin{array}{lll}-0.28231800 & 5.07546500 & -0.95141500\end{array}$ $\begin{array}{llll}0.79692900 & 4.78476600 & -2.78383200\end{array}$ $\begin{array}{lll}-1.17214500 & 5.19040600 & 0.99882800\end{array}$ $\begin{array}{lll}-0.96205300 & 5.80471500 & -1.38376200\end{array}$ $\begin{array}{lll}3.55410100 & -0.96078300 & 1.66659900\end{array}$ $\begin{array}{lll}4.23533000 & -1.71982400 & 0.69418100\end{array}$ $\begin{array}{lll}3.13257400 & -1.49738400 & 2.89942700\end{array}$ $\begin{array}{llll}4.48333800 & -3.06635200 & 0.98963800\end{array}$ $3.40889500 \quad-2.84902500 \quad 3.13971700$ $\begin{array}{lll}4.07579600 & -3.62704200 & 2.19697500\end{array}$ $\begin{array}{lll}5.00637600 & -3.68210100 & 0.26391400\end{array}$ $\begin{array}{lll}3.10083300 & -3.29594500 & 4.08051000\end{array}$ $\begin{array}{lll}4.28383400 & -4.67276200 & 2.40616800\end{array}$ $\begin{array}{lll}0.31787400 & 3.44742000 & 2.45512800\end{array}$ $\begin{array}{lll}1.09376200 & 2.72102900 & 2.71565400\end{array}$ $\begin{array}{lll}0.52821700 & 4.68249000 & 3.35329200\end{array}$ $\begin{array}{llll}0.46655000 & 4.39911100 & 4.41020000\end{array}$ $\begin{array}{lll}1.50869300 & 5.14044100 & 3.18167500\end{array}$ $\begin{array}{lll}-0.23311700 & 5.44909400 & 3.17022600\end{array}$ $\begin{array}{lll}-1.04071400 & 2.77670100 & 2.73741500\end{array}$ $\begin{array}{lll}-1.87590000 & 3.45080700 & 2.51476200\end{array}$ $\begin{array}{lll}-1.16293600 & 1.87188800 & 2.13141700\end{array}$ $\begin{array}{lll}-1.11216700 & 2.49643000 & 3.79490400\end{array}$ $\begin{array}{lll}2.40007100 & -0.67509500 & 3.95560700\end{array}$ $\begin{array}{lll}3.19268600 & -0.61051500 & 5.27607200\end{array}$ $\begin{array}{lll}4.19521000 & -0.19665000 & 5.12129800\end{array}$ $\begin{array}{llll}2.67170100 & 0.02447600 & 6.00166100\end{array}$ $\begin{array}{lll}3.30900800 & -1.60272800 & 5.72637800\end{array}$ $\begin{array}{llll}4.70496000 & -1.13888300 & -0.63643200\end{array}$ $\begin{array}{lll}4.41518700 & -0.08413700 & -0.67199100\end{array}$ $\begin{array}{lll}2.69299000 & 2.96498000 & -2.09790300\end{array}$ $\begin{array}{llll}3.66162400 & 4.05033800 & -2.60880500\end{array}$ $\begin{array}{llll}3.14948500 & 4.78051800 & -3.24554100\end{array}$ $\begin{array}{llll}4.12333900 & 4.59813000 & -1.77973200\end{array}$ $\begin{array}{llll}4.46210400 & 3.59527900 & -3.20302100\end{array}$ $\begin{array}{lll}4.02092400 & -1.83616400 & -1.82874300\end{array}$ $\begin{array}{llll}2.93058900 & -1.74958900 & -1.76493200\end{array}$ $\begin{array}{llll}4.34554100 & -1.37990600 & -2.77097600\end{array}$ $\begin{array}{lll}4.27489400 & -2.90144200 & -1.87160700\end{array}$ $6.24047400 \quad-1.19570400-0.76429800$ $\begin{array}{lll}6.56092600 & -0.72579000 & -1.70096600\end{array}$ $\begin{array}{lll}6.73199700 & -0.67290400 & 0.06383500\end{array}$ $\begin{array}{llll}6.60576700 & -2.22874200 & -0.76943700\end{array}$ $\begin{array}{lll}0.97273000 & -1.20877300 & 4.18845900\end{array}$ $\begin{array}{llll}0.39922200 & -1.21055700 & 3.25453000\end{array}$ $\begin{array}{lll}0.98862900 & -2.23199400 & 4.58214700\end{array}$ 


$\begin{array}{lrrr}\mathrm{H} & 0.44681000 & -0.57974800 & 4.91640700 \\ \mathrm{H} & 2.30163700 & 0.35019000 & 3.58578600 \\ \mathrm{C} & 2.09005000 & 2.16300600 & -3.26774900 \\ \mathrm{H} & 2.88788400 & 1.71309300 & -3.86955200 \\ \mathrm{H} & 1.44506300 & 1.35602400 & -2.90206600 \\ \mathrm{H} & 1.49616900 & 2.80251900 & -3.93097100 \\ \mathrm{H} & 3.28008200 & 2.26302800 & -1.49788800 \\ \mathrm{C} & -1.69134500 & -3.52093800 & -1.73411100 \\ \mathrm{C} & -2.68377400 & -3.16372800 & -2.66171900 \\ \mathrm{C} & -1.55890900 & -4.89479600 & -1.43076200 \\ \mathrm{C} & -3.53280400 & -4.11237500 & -3.23395000 \\ \mathrm{H} & -2.78208800 & -2.12802800 & -2.97083000 \\ \mathrm{C} & -2.40243900 & -5.84916300 & -1.98021800 \\ \mathrm{H} & -0.77276800 & -5.21124600 & -0.74826200 \\ \mathrm{C} & -3.40410100 & -5.46531600 & -2.88879900 \\ \mathrm{H} & -4.27285000 & -3.79185000 & -3.95834800 \\ \mathrm{H} & -2.29467100 & -6.90329800 & -1.74384300 \\ \mathrm{O} & -4.17592400 & -6.47004700 & -3.37793800 \\ \mathrm{C} & -5.15933600 & -6.15941300 & -4.35751200 \\ \mathrm{H} & -4.70492200 & -5.72313800 & -5.25576200 \\ \mathrm{H} & -5.63256400 & -7.10785400 & -4.61509100 \\ \mathrm{H} & -5.91722400 & -5.47070600 & -3.96201300 \\ \mathrm{O} & -4.89428000 & -2.24220400 & 1.02676000 \\ \mathrm{O} & -2.73317500 & -2.62628400 & 1.13667700 \\ \mathrm{C} & -4.77393600 & -3.27130300 & 2.05149700 \\ \mathrm{H} & -4.97108700 & -2.80107200 & 3.01789400 \\ \mathrm{H} & -5.52215000 & -4.03464400 & 1.84036800 \\ \mathrm{C} & -3.32695400 & -3.73632400 & 1.87398800 \\ \mathrm{H} & -3.21995700 & -4.62523800 & 1.24803000 \\ \mathrm{H} & -2.76963700 & -3.84838000 & 2.80301300 \\ \mathrm{C} & -3.68632500 & -1.89867400 & 0.61335300 \\ \mathrm{H} & 0.17652100 & -3.00054800 & -0.79963700 \\ & & & \end{array}$

$\begin{array}{lll}3.94806600 & -1.89741800 & 0.80193700\end{array}$ $3.06697900 \quad-1.36854300 \quad 3.05607500$ $\begin{array}{lll}4.13106500 & -3.22193700 & 1.21911700\end{array}$ $3.27444800 \quad-2.70593400 \quad 3.41642800$ $\begin{array}{lll}3.79884700 & -3.62387900 & 2.51014600\end{array}$ $\begin{array}{lll}4.54458700 & -3.94636400 & 0.52382100\end{array}$ $\begin{array}{llll}3.02587300 & -3.03038100 & 4.42275000\end{array}$ $\begin{array}{lll}3.95621300 & -4.65529800 & 2.81342400\end{array}$ $\begin{array}{lll}0.56905300 & 3.70567700 & 2.34117600\end{array}$ $\begin{array}{lll}1.27390300 & 2.90927700 & 2.59994200\end{array}$ $\begin{array}{lll}1.04181500 & 4.98738800 & 3.05662700\end{array}$ $\begin{array}{lll}1.07117700 & 4.82975400 & 4.14070200\end{array}$ $\begin{array}{lll}2.04477100 & 5.28378000 & 2.72957000\end{array}$ $\begin{array}{lll}0.36671100 & 5.82797500 & 2.86043900\end{array}$ $\begin{array}{lll}-0.81663800 & 3.26391300 & 2.85095900\end{array}$ $\begin{array}{lll}-1.58048500 & 4.02541200 & 2.65716300\end{array}$ $\begin{array}{lll}-1.13742100 & 2.33284300 & 2.37052100\end{array}$ $\begin{array}{lll}-0.78435900 & 3.09677800 & 3.93360700\end{array}$ $\begin{array}{lll}2.49775900 & -0.38815300 & 4.07747100\end{array}$ $\begin{array}{lll}3.43334400 & -0.23359300 & 5.29296100\end{array}$ $\begin{array}{llll}4.43555300 & 0.08844300 & 4.98920100\end{array}$ $\begin{array}{llll}3.03298700 & 0.51270100 & 5.98839300\end{array}$ $\begin{array}{lll}3.53960800 & -1.17583600 & 5.84225300\end{array}$ $\begin{array}{llll}4.33729000 & -1.48955000 & -0.61594200\end{array}$ $\begin{array}{lll}4.11335800 & -0.42532900 & -0.74032300\end{array}$ $\begin{array}{llll}2.41541000 & 2.56569900 & -2.34034100\end{array}$ $\begin{array}{llll}3.39815300 & 3.51383400 & -3.05645000\end{array}$ $\begin{array}{lll}2.87396500 & 4.21146100 & -3.71927700\end{array}$ $3.97727300 \quad 4.10724100-2.34026200$ $\begin{array}{lll}4.10175200 & 2.93899500 & -3.66911100\end{array}$ $\begin{array}{lll}3.51102500 & -2.25232500 & -1.66985500\end{array}$ $\begin{array}{lll}2.43733900 & -2.08827800 & -1.52563800\end{array}$ $\begin{array}{lll}3.77627300 & -1.91286600 & -2.67762700\end{array}$ $\begin{array}{llll}3.69762400 & -3.33128700 & -1.62411100\end{array}$ $\begin{array}{llll}5.84939000 & -1.66902900 & -0.85829300\end{array}$ $\begin{array}{lll}6.11694700 & -1.31828500 & -1.86136300\end{array}$ $\begin{array}{llll}6.44291000 & -1.10366700 & -0.13120400\end{array}$ $\begin{array}{lll}6.14710200 & -2.72100000 & -0.78447300\end{array}$ $\begin{array}{lll}1.07679400 & -0.79328400 & 4.51639400\end{array}$ $\begin{array}{lll}0.40052100 & -0.85698500 & 3.65622100\end{array}$ $\begin{array}{lll}1.07531900 & -1.76544200 & 5.02297400\end{array}$ $\begin{array}{lll}0.67076600 & -0.05405700 & 5.21636900\end{array}$ $\begin{array}{llll}2.41832400 & 0.59495900 & 3.60289400\end{array}$ $\begin{array}{lll}1.64055800 & 1.70000500 & -3.35318800\end{array}$ $\begin{array}{llll}2.33890900 & 1.12521400 & -3.97205000\end{array}$ $\begin{array}{lll}0.97615100 & 0.99334000 & -2.84299400\end{array}$ $\begin{array}{lll}1.03072900 & 2.31402000 & -4.02570300\end{array}$ $\begin{array}{llll}3.01210400 & 1.88374100 & -1.72632800\end{array}$ $\begin{array}{lll}-1.99178400 & -3.24781600 & -1.49614000\end{array}$ $-2.56444700 \quad-2.67120400 \quad-2.63935800$ $\begin{array}{lll}-1.84756800 & -4.65020300 & -1.47669300\end{array}$ $\begin{array}{lll}-2.99240600 & -3.45214000 & -3.71242800\end{array}$ $\begin{array}{lll}-2.67014700 & -1.59399700 & -2.71265800\end{array}$ $-2.27009200 \quad-5.43957800 \quad-2.53391200$ $\begin{array}{lll}-1.38231800 & -5.12692500 & -0.61648000\end{array}$ $\begin{array}{lll}-2.85495100 & -4.84713400 & -3.66519200\end{array}$ $\begin{array}{lll}-3.41717800 & -2.96445200 & -4.58210900\end{array}$ $\begin{array}{lll}-2.15178800 & -6.51821400 & -2.51589400\end{array}$ $\begin{array}{lll}-3.23476600 & -5.69918900 & -4.64916300\end{array}$ $\begin{array}{lll}-3.82243400 & -5.16394700 & -5.82919700\end{array}$ $\begin{array}{lll}-3.12886600 & -4.49426700 & -6.35315500\end{array}$ $\begin{array}{llll}-4.04614800 & -6.02179500 & -6.46454400\end{array}$ $\begin{array}{lll}-4.75157800 & -4.62498300 & -5.60479100\end{array}$ $\begin{array}{lll}-3.99278300 & -3.51026500 & 0.65631500\end{array}$ $\begin{array}{lll}-2.84339200 & -2.27924200 & 2.11417500\end{array}$ $\begin{array}{lll}-3.93092100 & -4.30971400 & 1.86389600\end{array}$ $\begin{array}{lll}-4.91336200 & -4.27008700 & 2.34271000\end{array}$ 


$\begin{array}{llll}\mathrm{H} & -3.69357300 & -5.33681100 & 1.58420600 \\ \mathrm{C} & -2.83570100 & -3.60779800 & 2.67241100 \\ \mathrm{H} & -1.84760000 & -4.05778100 & 2.52510800 \\ \mathrm{H} & -3.04931800 & -3.51985100 & 3.73781300 \\ \mathrm{C} & -3.27436200 & -2.38618300 & 0.82795700 \\ \mathrm{H} & -0.73415800 & -3.04525500 & 0.22271200\end{array}$
Imaginary Frequency $=-321.462800$ $\begin{array}{llll}3.03912800 & 3.49984900 & -2.86081100\end{array}$ $\begin{array}{llll}2.61683300 & 4.45264000 & -3.19927000\end{array}$ $\begin{array}{llll}3.78647500 & 3.72241600 & -2.09115700\end{array}$ $\begin{array}{llll}3.55473000 & 3.04298000 & -3.71289100\end{array}$ $2.08061400 \quad-2.46671700 \quad-3.24440900$ $\begin{array}{lll}1.05912400 & -2.24175300 & -2.91837100\end{array}$ $\begin{array}{lll}2.29647800 & -1.87149900 & -4.13911600\end{array}$ $\begin{array}{lll}2.11420800 & -3.52289500 & -3.53443100\end{array}$ $\begin{array}{lll}4.54085800 & -2.45381400 & -2.60529100\end{array}$ $\begin{array}{lll}4.77443500 & -1.88404400 & -3.51182400\end{array}$ $\begin{array}{lll}5.27806000 & -2.18960600 & -1.83891400\end{array}$ $\begin{array}{lll}4.67010500 & -3.51693400 & -2.83750600\end{array}$ $\begin{array}{lll}0.64769300 & -2.53150700 & 3.51068800\end{array}$ $\begin{array}{lll}-0.16021900 & -2.29558500 & 2.80929800\end{array}$ $\begin{array}{llll}0.56980600 & -3.59337700 & 3.76988200\end{array}$ $\begin{array}{llll}0.48326200 & -1.95593400 & 4.42877100\end{array}$ $\begin{array}{lll}2.03890800 & -1.11333700 & 2.72730100\end{array}$ $\begin{array}{llll}0.93062900 & 2.19875700 & -3.43343500\end{array}$ $\begin{array}{llll}1.44551900 & 1.74213700 & -4.28625600\end{array}$ $\begin{array}{llll}0.17910000 & 1.48934900 & -3.06854800\end{array}$ $\begin{array}{llll}0.40622900 & 3.08786800 & -3.80122800\end{array}$ $\begin{array}{llll}2.43682300 & 1.62060100 & -2.03402800\end{array}$ $-2.24766700 \quad-3.26144400-1.22027400$ $\begin{array}{lll}-1.92388600 & -4.16216300 & -2.23986000\end{array}$ $-2.03047500 \quad-3.66096100 \quad 0.11143600$ $\begin{array}{lll}-1.38937700 & -5.42242000 & -1.96121200\end{array}$ $\begin{array}{lll}-2.09377500 & -3.88290200 & -3.27718400\end{array}$ $\begin{array}{lll}-1.49703200 & -4.90722000 & 0.40487800\end{array}$ $\begin{array}{lll}-2.29701100 & -2.99250500 & 0.92508700\end{array}$ $\begin{array}{lll}-1.16623500 & -5.79824900 & -0.63023400\end{array}$ $\begin{array}{lll}-1.15115900 & -6.09143900 & -2.77984200\end{array}$ $\begin{array}{lll}-1.32848400 & -5.21996300 & 1.43009000\end{array}$ $\begin{array}{lll}-0.64090400 & -6.98916800 & -0.23834600\end{array}$ $\begin{array}{lll}-0.36631700 & -7.97108100 & -1.23044900\end{array}$ $\begin{array}{lll}0.39979100 & -7.62667600 & -1.93703100\end{array}$ $\begin{array}{lll}0.00538200 & -8.84354700 & -0.69125400\end{array}$ $\begin{array}{llll}-1.27305200 & -8.24523100 & -1.78407800\end{array}$ $\begin{array}{lll}-5.10168600 & -2.29664600 & -2.53597400\end{array}$ $\begin{array}{lll}-4.91889600 & -2.57621900 & -0.28057100\end{array}$ $-6.09180800 \quad-3.25861200 \quad-2.14071800$ $\begin{array}{lll}-7.07180700 & -2.77558900 & -2.03845200\end{array}$ $\begin{array}{lll}-6.14508400 & -4.02863700 & -2.91350900\end{array}$ $\begin{array}{lll}-5.55580800 & -3.75257500 & -0.80097900\end{array}$ $\begin{array}{lll}-4.82661500 & -4.56106700 & -0.92316400\end{array}$ $\begin{array}{lll}-6.32975200 & -4.05219700 & -0.09144700\end{array}$ $\begin{array}{lll}-4.44104800 & -1.83138800 & -1.38356400\end{array}$ $\begin{array}{lll}-2.69177100 & -1.71780000 & -2.61817300\end{array}$

$\begin{array}{lrrr}\text { TS3a } & & & \\ \text { C } & -3.25144400 & 0.27249400 & -0.72293600 \\ \text { C } & -4.57190500 & -0.18599100 & -0.66774400 \\ \text { C } & -5.63860300 & 0.69575700 & -0.58732800 \\ \text { C } & -5.36045700 & 2.06914600 & -0.55184500 \\ \text { C } & -4.04261500 & 2.53401800 & -0.60583000 \\ \text { C } & -2.97165000 & 1.63816200 & -0.69889200 \\ \text { C } & -2.29264200 & -0.86719300 & -0.83662700 \\ \text { C } & -3.07288800 & -2.05979500 & -0.88825100 \\ \text { H } & -6.66348500 & 0.33921200 & -0.54641600 \\ \text { H } & -6.17853700 & 2.78002000 & -0.48097400 \\ \text { H } & -3.84843000 & 3.60214200 & -0.58057500 \\ \text { H } & -1.94861200 & 1.99867400 & -0.75344400 \\ \text { C } & 1.76199700 & -0.11200300 & -0.65950300 \\ \text { C } & 3.66454600 & 1.11125100 & -0.75510800 \\ \text { C } & 4.01282200 & -0.16018000 & -0.43091500 \\ \text { H } & 4.25955800 & 1.99878100 & -0.90167200\end{array}$




\begin{tabular}{|c|c|c|c|}
\hline $\mathrm{H}$ & 4.97362300 & -0.60925500 & -0.23501800 \\
\hline $\mathrm{N}$ & 2.83615200 & -0.89701000 & -0.37589100 \\
\hline $\mathrm{N}$ & 2.28355800 & 1.12433000 & -0.89018900 \\
\hline $\mathrm{Au}$ & -0.24256200 & -0.58156400 & -0.75515400 \\
\hline $\mathrm{C}$ & 1.51585400 & 2.30695000 & -1.22566100 \\
\hline $\mathrm{C}$ & 1.26486700 & 2.58305500 & -2.58494200 \\
\hline $\mathrm{C}$ & 1.08114500 & 3.14384200 & -0.17766600 \\
\hline $\mathrm{C}$ & 0.53390300 & 3.74138400 & -2.87824200 \\
\hline $\mathrm{C}$ & 0.35798800 & 4.29001400 & -0.53323500 \\
\hline $\mathrm{C}$ & 0.08458200 & 4.58609700 & -1.86660800 \\
\hline $\mathrm{H}$ & 0.32047900 & 3.98815500 & -3.91398100 \\
\hline $\mathrm{H}$ & 0.01217700 & 4.96309800 & 0.24566100 \\
\hline $\mathrm{H}$ & -0.47349900 & 5.48349800 & -2.11882300 \\
\hline $\mathrm{C}$ & 2.80369000 & -2.30695700 & -0.04533100 \\
\hline $\mathrm{C}$ & 2.93147000 & -3.24561900 & -1.08862300 \\
\hline $\mathrm{C}$ & 2.70672100 & -2.67372500 & 1.31232700 \\
\hline $\mathrm{C}$ & 2.96633000 & -4.59942300 & -0.73034900 \\
\hline $\mathrm{C}$ & 2.74332400 & -4.04228500 & 1.60962500 \\
\hline $\mathrm{C}$ & 2.87963000 & -4.99472800 & 0.60234500 \\
\hline $\mathrm{H}$ & 3.07367100 & -5.35156000 & -1.50626000 \\
\hline $\mathrm{H}$ & 2.68152400 & -4.36361300 & 2.64524000 \\
\hline $\mathrm{H}$ & 2.93164700 & -6.04959100 & 0.85945600 \\
\hline $\mathrm{C}$ & 1.38359600 & 2.86484700 & 1.29211600 \\
\hline $\mathrm{H}$ & 1.88738400 & 1.89546200 & 1.36070900 \\
\hline $\mathrm{C}$ & 2.34500200 & 3.92312800 & 1.87069300 \\
\hline $\mathrm{H}$ & 2.59272200 & 3.68434900 & 2.91106200 \\
\hline $\mathrm{H}$ & 3.28001700 & 3.97099900 & 1.30141300 \\
\hline $\mathrm{H}$ & 1.89549600 & 4.92237900 & 1.85561500 \\
\hline $\mathrm{C}$ & 0.09789400 & 2.76651900 & 2.13610900 \\
\hline $\mathrm{H}$ & -0.44797500 & 3.71655400 & 2.15509200 \\
\hline $\mathrm{H}$ & -0.57853800 & 1.99687600 & 1.74803900 \\
\hline $\mathrm{H}$ & 0.34629300 & 2.51032800 & 3.17218700 \\
\hline $\mathrm{C}$ & 2.58242100 & -1.65653700 & 2.44327100 \\
\hline $\mathrm{C}$ & 3.77962000 & -1.73865000 & 3.41125700 \\
\hline $\mathrm{H}$ & 4.73095100 & -1.59907500 & 2.88605300 \\
\hline $\mathrm{H}$ & 3.69876200 & -0.96193900 & 4.17977400 \\
\hline $\mathrm{H}$ & 3.82038000 & -2.70714900 & 3.92205800 \\
\hline $\mathrm{C}$ & 3.03088500 & -2.84706700 & -2.55802900 \\
\hline $\mathrm{H}$ & 3.02481300 & -1.75421000 & -2.61778100 \\
\hline $\mathrm{C}$ & 1.76137500 & 1.69295800 & -3.72067700 \\
\hline $\mathrm{C}$ & 2.75399100 & 2.44507800 & -4.62966000 \\
\hline $\mathrm{H}$ & 2.27610900 & 3.29155600 & -5.13524100 \\
\hline $\mathrm{H}$ & 3.60386000 & 2.83539800 & -4.05886200 \\
\hline $\mathrm{H}$ & 3.14383500 & 1.77382100 & -5.40312600 \\
\hline $\mathrm{C}$ & 1.81047500 & -3.35079700 & -3.35406100 \\
\hline $\mathrm{H}$ & 0.87562300 & -2.97089700 & -2.92660700 \\
\hline $\mathrm{H}$ & 1.87296200 & -3.01659100 & -4.39593800 \\
\hline $\mathrm{H}$ & 1.75836400 & -4.44549900 & -3.35844200 \\
\hline $\mathrm{C}$ & 4.34951600 & -3.33074500 & -3.19293200 \\
\hline $\mathrm{H}$ & 4.42081100 & -2.98112600 & -4.22883800 \\
\hline $\mathrm{H}$ & 5.22072700 & -2.95285400 & -2.64650000 \\
\hline $\mathrm{H}$ & 4.41436500 & -4.42442700 & -3.20846600 \\
\hline $\mathrm{C}$ & 1.24700200 & -1.81182500 & 3.19740000 \\
\hline $\mathrm{H}$ & 0.39257900 & -1.69171700 & 2.52129800 \\
\hline $\mathrm{H}$ & 1.16561100 & -2.79451300 & 3.67577000 \\
\hline $\mathrm{H}$ & 1.16520800 & -1.05327300 & 3.98401800 \\
\hline $\mathrm{H}$ & 2.58843500 & -0.65387400 & 2.00472400 \\
\hline $\mathrm{C}$ & 0.59090500 & 1.11015400 & -4.53657200 \\
\hline $\mathrm{H}$ & 0.96955900 & 0.44222800 & -5.31856700 \\
\hline $\mathrm{H}$ & -0.08971200 & 0.53690900 & -3.89674400 \\
\hline $\mathrm{H}$ & 0.00909700 & 1.89861700 & -5.02694700 \\
\hline $\mathrm{H}$ & 2.30089300 & 0.84685900 & -3.28312100 \\
\hline $\mathrm{C}$ & -2.59145400 & -3.44157200 & -0.89753500 \\
\hline $\mathrm{C}$ & -1.50903900 & -3.83029500 & -0.08869600 \\
\hline $\mathrm{C}$ & -3.21085000 & -4.42342000 & -1.70681300 \\
\hline $\mathrm{C}$ & -1.06148200 & -5.14757100 & -0.06277600 \\
\hline $\mathrm{H}$ & 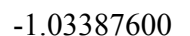 & -3.09 & $0.55527 \mathrm{C}$ \\
\hline
\end{tabular}

$\begin{array}{lrrr}\mathrm{C} & -2.75827500 & -5.72980500 & -1.70503200 \\ \mathrm{H} & -4.02983700 & -4.13775400 & -2.35696400 \\ \mathrm{C} & -1.68354300 & -6.10836300 & -0.87627800 \\ \mathrm{H} & -0.23487600 & -5.41148100 & 0.58529700 \\ \mathrm{H} & -3.21393400 & -6.48564100 & -2.33624400 \\ \mathrm{O} & -1.33271800 & -7.40945700 & -0.93670600 \\ \mathrm{C} & -0.26693400 & -7.87906700 & -0.11460800 \\ \mathrm{H} & -0.50015500 & -7.74841500 & 0.94891900 \\ \mathrm{H} & -0.16850700 & -8.94160600 & -0.33835500 \\ \mathrm{H} & 0.67199400 & -7.36451700 & -0.35166300 \\ \mathrm{O} & -5.39699700 & -2.24897500 & -1.74454900 \\ \mathrm{O} & -5.03959500 & -2.23429200 & 0.51157200 \\ \mathrm{C} & -6.48504200 & -2.96156500 & -1.12869600 \\ \mathrm{H} & -7.35887400 & -2.30568000 & -1.03523500 \\ \mathrm{H} & -6.73500400 & -3.81300400 & -1.76552500 \\ \mathrm{C} & -5.90533300 & -3.34384500 & 0.22883300 \\ \mathrm{H} & -5.33530400 & -4.27860900 & 0.18449200 \\ \mathrm{H} & -6.64311700 & -3.39785900 & 1.03209300 \\ \mathrm{C} & -4.59506900 & -1.70040800 & -0.72022200 \\ \mathrm{H} & -2.53814000 & -1.41506100 & -1.96389200 \\ \mathrm{Imaginary} & \text { Frequency }=-822.644900 & \end{array}$

$\begin{array}{cccc}\text { Int4a } & & & \\ \mathrm{C} & -3.21837700 & 0.02398600 & -1.67626400 \\ \mathrm{C} & -3.63173000 & -0.47414800 & -0.42995700 \\ \mathrm{C} & -4.35185700 & 0.32303000 & 0.44552400 \\ \mathrm{C} & -4.69855900 & 1.62154700 & 0.03772900 \\ \mathrm{C} & -4.31319900 & 2.10504600 & -1.21532600 \\ \mathrm{C} & -3.55452000 & 1.31170700 & -2.08530900 \\ \mathrm{C} & -2.40585800 & -1.00894200 & -2.35055000 \\ \mathrm{C} & -2.32156700 & -2.15914400 & -1.57316600 \\ \mathrm{H} & -4.64869700 & -0.03841900 & 1.42552900 \\ \mathrm{H} & -5.27710400 & 2.25516100 & 0.70353800 \\ \mathrm{H} & -4.59715900 & 3.10958500 & -1.51469900 \\ \mathrm{H} & -3.23624600 & 1.69599500 & -3.05049700 \\ \mathrm{C} & 1.57515200 & -0.32198200 & -0.80545600 \\ \mathrm{C} & 3.42307800 & 0.94925400 & -0.58377400 \\ \mathrm{C} & 3.68406000 & -0.25618400 & -0.01488300 \\ \mathrm{H} & 4.02994800 & 1.83416000 & -0.69217200 \\ \mathrm{H} & 4.56552100 & -0.63857700 & 0.47479100 \\ \mathrm{~N} & 2.53855200 & -1.02706800 & -0.15966100 \\ \mathrm{~N} & 2.12259000 & 0.89306400 & -1.06549300 \\ \mathrm{Au} & -0.29423200 & -0.93750900 & -1.31262100 \\ \mathrm{C} & 1.46283700 & 1.98538500 & -1.75466000 \\ \mathrm{C} & 1.58336000 & 2.06272100 & -3.15703400 \\ \mathrm{C} & 0.75954300 & 2.93466000 & -0.98664300 \\ \mathrm{C} & 0.95061400 & 3.13839000 & -3.79265800 \\ \mathrm{C} & 0.15034500 & 3.98882900 & -1.67884500 \\ \mathrm{C} & 0.24185800 & 4.09065600 & -3.06446500 \\ \mathrm{H} & 1.02107100 & 3.23519000 & -4.87194200 \\ \mathrm{H} & -0.39813400 & 4.74257500 & -1.12237900 \\ \mathrm{H} & -0.23444500 & 4.92053200 & -3.57900900 \\ \mathrm{C} & 2.41768200 & -2.39234900 & 0.31446700 \\ \mathrm{C} & 2.77827300 & -3.43808900 & -0.55886800 \\ \mathrm{C} & 1.98822600 & -2.60505900 & 1.64010300 \\ \mathrm{C} & 2.68174600 & -4.74423100 & -0.06265600 \\ \mathrm{C} & 1.91312200 & -3.93307000 & 2.07888100 \\ \mathrm{C} & 2.25421600 & -4.99077300 & 1.23936700 \\ \mathrm{H} & 2.95303100 & -5.57777500 & -0.70324500 \\ \mathrm{H} & 1.59342200 & -4.13983300 & 3.09573800 \\ \mathrm{H} & 2.19620900 & -6.01211600 & 1.60554400 \\ \mathrm{C} & 0.66315800 & 2.86916600 & 0.53477600 \\ \mathrm{H} & 1.13184800 & 1.93787700 & 0.86912300 \\ \mathrm{C} & 1.43735800 & 4.03217200 & 1.18860900 \\ \mathrm{H} & 1.40036000 & 3.94749700 & 2.28048300\end{array}$




\begin{tabular}{|c|c|c|c|}
\hline $\mathrm{H}$ & 2.48995700 & 4.03735300 & 0.88386200 \\
\hline $\mathrm{H}$ & 1.00759400 & 5.00211500 & 0.91419900 \\
\hline $\mathrm{C}$ & -0.79960600 & 2.83627200 & 1.01745000 \\
\hline $\mathrm{H}$ & -1.33293800 & 3.75695800 & 0.75577200 \\
\hline $\mathrm{H}$ & -1.34817400 & 1.99569900 & 0.57967300 \\
\hline $\mathrm{H}$ & -0.83236100 & 2.73561400 & 2.10823500 \\
\hline $\mathrm{C}$ & 1.65280400 & -1.46591000 & 2.59857200 \\
\hline $\mathrm{C}$ & 2.71899200 & -1.34963800 & 3.70745500 \\
\hline $\mathrm{H}$ & 3.72156900 & -1.20094700 & 3.29071800 \\
\hline $\mathrm{H}$ & 2.49455300 & -0.50106500 & 4.36330600 \\
\hline $\mathrm{H}$ & 2.74829400 & -2.25300200 & 4.32716500 \\
\hline $\mathrm{C}$ & 3.28914700 & -3.20097400 & -1.97752700 \\
\hline $\mathrm{H}$ & 3.21407000 & -2.13057800 & -2.19548100 \\
\hline $\mathrm{C}$ & 2.37344600 & 1.05183700 & -3.98321900 \\
\hline $\mathrm{C}$ & 3.57872000 & 1.71579300 & -4.67920200 \\
\hline $\mathrm{H}$ & 3.25824800 & 2.47064800 & -5.40590800 \\
\hline $\mathrm{H}$ & 4.23772600 & 2.20903500 & -3.95617600 \\
\hline $\mathrm{H}$ & 4.16710800 & 0.96466800 & -5.21786400 \\
\hline $\mathrm{C}$ & 2.43705400 & -3.93568400 & -3.03016900 \\
\hline $\mathrm{H}$ & 1.38341900 & -3.64415800 & -2.96767500 \\
\hline $\mathrm{H}$ & 2.80080600 & -3.69924700 & -4.03672400 \\
\hline $\mathrm{H}$ & 2.49053000 & -5.02310300 & -2.90775900 \\
\hline $\mathrm{C}$ & 4.77669300 & -3.59054600 & -2.09983100 \\
\hline $\mathrm{H}$ & 5.14476400 & -3.37542000 & -3.10927200 \\
\hline $\mathrm{H}$ & 5.39816200 & -3.03753700 & -1.38660300 \\
\hline $\mathrm{H}$ & 4.92518200 & -4.65951200 & -1.91070000 \\
\hline $\mathrm{C}$ & 0.24172400 & -1.60759800 & 3.20039400 \\
\hline $\mathrm{H}$ & -0.52316200 & -1.66954400 & 2.41973000 \\
\hline $\mathrm{H}$ & 0.15996600 & -2.50026300 & 3.83140000 \\
\hline $\mathrm{H}$ & 0.01855500 & -0.74086200 & 3.83314000 \\
\hline $\mathrm{H}$ & 1.66637400 & -0.52825400 & 2.03337700 \\
\hline $\mathrm{C}$ & 1.47609200 & 0.32510800 & -5.00381300 \\
\hline $\mathrm{H}$ & 2.05666500 & -0.42443400 & -5.55342200 \\
\hline $\mathrm{H}$ & 0.64463400 & -0.18717900 & -4.50571300 \\
\hline $\mathrm{H}$ & 1.05327300 & 1.02022000 & -5.73783500 \\
\hline $\mathrm{H}$ & 2.77140200 & 0.28889600 & -3.30630300 \\
\hline $\mathrm{C}$ & -1.90970900 & -3.50324600 & -2.00506400 \\
\hline $\mathrm{C}$ & -2.10827800 & -3.92808700 & -3.32870500 \\
\hline $\mathrm{C}$ & -1.32417400 & -4.41119500 & -1.09627500 \\
\hline $\mathrm{C}$ & -1.74715900 & -5.20788800 & -3.74378600 \\
\hline $\mathrm{H}$ & -2.58801500 & -3.26352200 & -4.04163100 \\
\hline $\mathrm{C}$ & -0.95641800 & -5.68343900 & -1.49855000 \\
\hline $\mathrm{H}$ & -1.13089500 & -4.09268800 & -0.07752000 \\
\hline $\mathrm{C}$ & -1.16705600 & -6.09809900 & -2.82665200 \\
\hline $\mathrm{H}$ & -1.93574200 & -5.50637000 & -4.76821500 \\
\hline $\mathrm{H}$ & -0.49706700 & -6.38105300 & -0.80617000 \\
\hline $\mathrm{O}$ & -0.78081000 & -7.36181200 & -3.11552400 \\
\hline $\mathrm{C}$ & -0.99058100 & -7.86208000 & -4.43363700 \\
\hline $\mathrm{H}$ & -0.43207200 & -7.27796000 & -5.17532900 \\
\hline $\mathrm{H}$ & -0.61816000 & -8.88690500 & -4.42329000 \\
\hline $\mathrm{H}$ & -2.05605000 & -7.86357800 & -4.69326800 \\
\hline $\mathrm{O}$ & -4.28581800 & -2.82954500 & -0.41165900 \\
\hline $\mathrm{O}$ & -2.55934800 & -2.21327700 & 0.94111500 \\
\hline $\mathrm{C}$ & -4.66808600 & -3.19057400 & 0.91678500 \\
\hline $\mathrm{H}$ & -5.35978100 & -2.44940500 & 1.33888200 \\
\hline $\mathrm{H}$ & -5.16302300 & -4.16235100 & 0.86890100 \\
\hline $\mathrm{C}$ & -3.32593300 & -3.20782600 & 1.64527700 \\
\hline $\mathrm{H}$ & -2.83204200 & -4.18255300 & 1.56146400 \\
\hline $\mathrm{H}$ & -3.38731700 & -2.91674500 & 2.69672100 \\
\hline $\mathrm{C}$ & -3.19628700 & -1.93335500 & -0.29641800 \\
\hline $\mathrm{H}$ & -2.10941200 & -0.94847800 & -3.39434700 \\
\hline
\end{tabular}

$\begin{array}{rrr}-4.15351300 & 0.46378500 & 0.50408000 \\ -4.46629000 & 1.77246600 & 0.09584400 \\ -4.37462400 & 2.13441200 & -1.24862900 \\ -3.96771400 & 1.20467600 & -2.21750700 \\ -3.21253700 & -1.26644800 & -2.56871600 \\ -3.04921000 & -2.33650700 & -1.76063700 \\ -4.21485200 & 0.18682600 & 1.55313900 \\ -4.77785200 & 2.50713700 & 0.83332300 \\ -4.61789100 & 3.15009200 & -1.54967400 \\ -3.89392700 & 1.49437100 & -3.26275500 \\ -2.56783800 & -3.66821200 & -2.15303200 \\ -1.54487600 & -3.79727100 & -3.10599400 \\ -3.11935800 & -4.85584400 & -1.62675800 \\ -1.08284700 & -5.04427500 & -3.52874200 \\ -1.08266700 & -2.90067100 & -3.50947100 \\ -2.66700300 & -6.10212900 & -2.03653400 \\ -3.93332400 & -4.78778200 & -0.91437400 \\ -1.64395900 & -6.20896500 & -2.99100300 \\ -0.28421100 & -5.09393200 & -4.26032400 \\ -3.10039000 & -7.01506200 & -1.63940000 \\ -1.26999100 & -7.47863300 & -3.32272800 \\ -0.25214900 & -7.64625300 & -4.29554500 \\ 0.69743000 & -7.20176200 & -3.96850600 \\ -0.12344100 & -8.72393600 & -4.41258400 \\ -0.53980200 & -7.20867000 & -5.26087500 \\ -4.41986200 & -2.73157700 & 0.23841500 \\ -2.25729000 & -2.05413000 & 0.56906700 \\ -4.04111100 & -3.10664000 & 1.56260100 \\ -4.39673100 & -2.36831600 & 2.29558200 \\ -4.49084200 & -4.07874300 & 1.78314300 \\ -2.51924900 & -3.12456800 & 1.47317900 \\ -2.15003800 & -4.07765200 & 1.07194700 \\ -2.01274900 & -2.90411300 & 2.41710000 \\ -3.37508500 & -1.92168600 & -0.30682400 \\ -3.05673300 & -1.26570900 & -3.64305800 \\ & & \end{array}$

$\begin{array}{lll}-4.15351300 & 0.46378500 & 0.50408000\end{array}$ $\begin{array}{lll}-4.46629000 & 1.77246600 & 0.09584400\end{array}$ $-3.96771400$ $\begin{array}{lll}-3.21253700 & -1.26644800 & -2.56871600\end{array}$ $\begin{array}{lll}-4.21485200 & 0.18682600 & 1.55313900\end{array}$ $\begin{array}{lll}-4.77785200 & 2.50713700 & 0.83332300\end{array}$ $\begin{array}{lll}-4.61789100 & 3.15009200 & -1.54967400\end{array}$ $-2.56783800$ $\begin{array}{lll}-1.54487600 & -3.79727100 & -3.10599400\end{array}$ $\begin{array}{lll}-3.11935800 & -4.85584400 & -1.62675800\end{array}$ $\begin{array}{lll}-1.08284700 & -5.04427500 & -3.52874200\end{array}$ $\begin{array}{llll}-2.66700300 & -6.10212900 & -2.03653400\end{array}$ $\begin{array}{lll}-3.93332400 & -4.78778200 & -0.91437400\end{array}$ $\begin{array}{lll}-1.64395900 & -6.20896500 & -2.99100300\end{array}$ $\begin{array}{lll}-1.26999100 & -7.47863300 & -3.32272800\end{array}$ $-0.25214900-7.64625300 \quad-4.29554500$ $-0.53980200$ $\begin{array}{lll}-4.41986200 & -2.73157700 & 0.23841500\end{array}$ $\begin{array}{lll}-2.25729000 & -2.05413000 & 0.56906700\end{array}$ $\begin{array}{llll}-4.49084200 & -4.07874300 & 1.78314300\end{array}$ $\begin{array}{lll}-2.51924900 & -3.12456800 & 1.47317900\end{array}$ $\begin{array}{lll}-5.51924900 & -3.12456800 & 1.47317900\end{array}$ $\begin{array}{lll}-3.05673300 & -1.26570900 & -3.64305800\end{array}$ 


$\begin{array}{lrrr}\mathrm{C} & 3.26616500 & -0.53781700 & -1.54009200 \\ \mathrm{H} & 3.00950400 & -2.65720300 & -1.15561000 \\ \mathrm{H} & 3.23484100 & 1.59520700 & -1.76169300 \\ \mathrm{O} & 4.49503800 & -0.56735500 & -2.13493900 \\ \mathrm{C} & 5.13782000 & -1.82268900 & -2.28641900 \\ \mathrm{H} & 6.09205300 & -1.61363100 & -2.77367100 \\ \mathrm{H} & 5.32384900 & -2.30141900 & -1.31583600 \\ \mathrm{H} & 4.55104400 & -2.50434200 & -2.91655600 \\ \mathrm{C} & -1.45483000 & -0.88574000 & 0.87033400 \\ \mathrm{C} & -0.58745400 & -0.11890300 & 0.27417300 \\ \mathrm{Im} & \end{array}$

Imaginary Frequency $=-848.8530$

$\begin{array}{lccc}\text { TS2 } & & & \\ \text { C } & -4.75088800 & 1.14344200 & 2.57699000 \\ \text { C } & -3.49594300 & 0.57426600 & 2.70532400 \\ \text { C } & -2.68176200 & 0.40951400 & 1.55710300 \\ \text { C } & -3.23237400 & 0.74712200 & 0.28768600 \\ \text { C } & -4.49775700 & 1.33624500 & 0.16539900 \\ \text { C } & -5.25662100 & 1.52371100 & 1.31364500 \\ \text { H } & -5.36289000 & 1.30399300 & 3.46117200 \\ \text { H } & -3.09981800 & 0.29616100 & 3.67746000 \\ \text { H } & -4.88594500 & 1.61443700 & -0.81063000 \\ \text { H } & -6.25858500 & 1.93705500 & 1.23825300\end{array}$

$\begin{array}{rrr}-3.69318900 & 0.99583600 & 2.75252200 \\ -2.52483500 & 1.25386200 & 2.12118500 \\ -2.36460200 & 1.09626800 & 0.66904200 \\ -3.57341400 & 0.63493300 & -0.06910300 \\ -4.79038700 & 0.37385400 & 0.67717400 \\ -4.85577700 & 0.54386900 & 2.01939500 \\ -3.76501400 & 1.12767400 & 3.82930100 \\ -1.65329800 & 1.59222500 & 2.67323400 \\ -5.65728800 & 0.03332900 & 0.12015700 \\ -5.77917800 & 0.34038400 & 2.55377300 \\ -3.57929400 & 0.44515800 & -1.41792400 \\ -2.50034900 & 0.58944500 & -2.22239500 \\ 0.22978400 & 2.70974400 & -0.60986200 \\ -2.94358400 & 0.48592700 & -3.58598400 \\ -4.24887400 & -0.29493900 & -3.44425200 \\ -3.10047100 & 1.49254400 & -3.99135300 \\ -2.17027400 & -0.02862500 & -4.15859800 \\ -5.02403000 & -0.00829100 & -4.15697800 \\ -4.09031100 & -1.37972600 & -3.48225000 \\ -4.68532400 & 0.07999600 & -2.12833500 \\ 1.16384700 & 0.75279500 & -0.46087500 \\ 2.39115500 & 1.22878500 & -0.93643000 \\ 1.07238400 & -0.61757600 & -0.14656200 \\ 3.49657600 & 0.38881200 & -1.09787800 \\ 2.49030300 & 2.28288800 & -1.18713300 \\ 2.15817700 & -1.46365400 & -0.30074900 \\ 0.12923800 & -1.01036700 & 0.22361900 \\ 3.38272000 & -0.96744100 & -0.77865900 \\ 4.42846300 & 0.80133300 & -1.46810700 \\ 2.08854800 & -2.51963900 & -0.05715300 \\ 4.38986300 & -1.88557400 & -0.89441100 \\ 5.64990500 & -1.43821200 & -1.36304300 \\ 6.29689400 & -2.31765200 & -1.37288800 \\ 5.58200900 & -1.02779400 & -2.37984200 \\ 6.08268900 & -0.67793300 & -0.69875700 \\ -1.17909700 & 1.37597500 & 0.12905000 \\ 0.02269000 & 1.67778200 & -0.30587900\end{array}$


$\begin{array}{lrrr}\mathrm{H} & 5.27935400 & -0.06196100 & -2.22138800 \\ \mathrm{C} & -1.17685600 & 0.28460500 & 1.64923000 \\ \mathrm{C} & -0.72418600 & 0.71735100 & 0.27809400\end{array}$

TS3,

$\begin{array}{llll}\text { C } & -4.81056200 & 0.94900200 & 2.46471300\end{array}$

$\begin{array}{llll}\text { C } & -3.45951400 & 0.65169500 & 2.67367500\end{array}$

$\begin{array}{llll}\text { C } & -2.61734800 & 0.52627300 & 1.56904300\end{array}$

$\begin{array}{llll}\mathrm{C} & -3.12710200 & 0.70568100 & 0.27383400\end{array}$

$\begin{array}{llll}\text { C } & -4.46312800 & 1.00706500 & 0.05489800\end{array}$

$\begin{array}{llll}\text { C } & -5.30598500 & 1.12588800 & 1.16857000\end{array}$

$\begin{array}{llll}\mathrm{H} & -5.48305500 & 1.04431400 & 3.31319600\end{array}$

$\mathrm{H} \quad-3.06056000 \quad 0.51235000 \quad 3.67459600$

$\mathrm{H} \quad-4.85852400 \quad 1.14225300 \quad-0.94866900$

$\begin{array}{llll}\mathrm{H} & -6.35870100 & 1.35421000 & 1.02249600\end{array}$

C $\quad-2.00050700 \quad 0.52268900 \quad-0.72852600$

O $\quad-2.23950100 \quad-0.61319200 \quad-1.55269500$

$\mathrm{H} \quad-0.52091700 \quad 1.23068900 \quad 1.02130300$

$\begin{array}{llll}\mathrm{C} & -1.96645200 & -0.25402000 & -2.90737600\end{array}$

$\begin{array}{llll}\mathrm{C} & -2.22487700 & 1.24908400 & -2.90563000\end{array}$

$\mathrm{H} \quad-0.92730600 \quad-0.48735400 \quad-3.17174500$

$\mathrm{H} \quad-2.64432000 \quad-0.82162600 \quad-3.55089800$

$\mathrm{H} \quad-1.63396500 \quad 1.80835600 \quad-3.63587000$

$\mathrm{H} \quad-3.28977800 \quad 1.48018600 \quad-3.04776100$

$\begin{array}{lllll}\mathrm{O} & -1.79309000 & 1.63245800 & -1.59735000\end{array}$

$\begin{array}{lllll}\mathrm{C} & & 0.54337400 & -0.15001000 & -0.30525100\end{array}$

$\begin{array}{llll}\mathrm{C} & 1.14729400 & 0.47094700 & -1.40630400\end{array}$

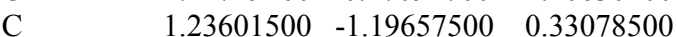

$\begin{array}{llll}\mathrm{C} & 2.39584100 & 0.06041400 & -1.87719600\end{array}$

$\mathrm{H} \quad 0.64699400 \quad 1.30452400 \quad-1.88803000$

$\begin{array}{llll}\mathrm{C} & 2.47527700 & -1.61581700 & -0.12821500\end{array}$

$\begin{array}{llll}\mathrm{H} & 0.78065800 & -1.66893900 & 1.19485000\end{array}$

C $\quad 3.06499100 \quad-0.99146800 \quad-1.23848500$

$\begin{array}{llll}\mathrm{H} & 2.83761200 & 0.57100000 & -2.72543600\end{array}$

$\mathrm{H} \quad 3.00980200 \quad-2.42856300 \quad 0.35364700$

$\begin{array}{llll}\mathrm{O} & 4.28209900 & -1.47720500 & -1.61336900\end{array}$

$\begin{array}{llll}\mathrm{C} & 4.93433200 & -0.88020000 & -2.72314200\end{array}$

$\mathrm{H} \quad 5.87582700 \quad-1.41942400 \quad-2.84191000$

$\mathrm{H} \quad 4.34126700 \quad-0.97982300 \quad-3.64185300$

$\begin{array}{llll}\mathrm{H} & 5.14533400 & 0.18222400 & -2.54367600\end{array}$

$\begin{array}{llll}\mathrm{C} & -1.16112800 & 0.18395500 & 1.59219000\end{array}$

$\begin{array}{lllll}\mathrm{C} & & -0.77889700 & 0.28148200 & 0.22543100\end{array}$

Imaginary Frequency $=-1026.9022$

\begin{tabular}{|c|c|c|c|}
\hline \multicolumn{4}{|l|}{ TS } \\
\hline $\mathrm{C}$ & -3.21301700 & -0.73431600 & 1.38287200 \\
\hline $\mathrm{C}$ & -4.50736300 & -1.25512100 & 1.51311900 \\
\hline $\mathrm{C}$ & -5.40544300 & -0.77981200 & 2.46027100 \\
\hline $\mathrm{C}$ & -4.99060100 & 0.26200300 & 3.29395900 \\
\hline $\mathrm{C}$ & -3.70171400 & 0.79698000 & 3.16862100 \\
\hline $\mathrm{C}$ & -2.80270800 & 0.31416600 & 2.21715000 \\
\hline $\mathrm{C}$ & -2.47347800 & -1.41801900 & 0.34083800 \\
\hline $\mathrm{C}$ & -1.41525900 & -1.59058700 & -0.39787600 \\
\hline $\mathrm{H}$ & -6.40204500 & -1.20102900 & 2.54712000 \\
\hline $\mathrm{H}$ & -5.67119900 & 0.65998900 & 4.04008400 \\
\hline $\mathrm{H}$ & -3.39346000 & 1.60584600 & 3.82470900 \\
\hline $\mathrm{H}$ & -1.80532200 & 0.72984000 & 2.12700500 \\
\hline $\mathrm{C}$ & 1.87606500 & 0.77372500 & 0.57541400 \\
\hline $\mathrm{C}$ & 3.36815400 & 2.47176300 & 0.62115500 \\
\hline $\mathrm{C}$ & 3.89615400 & 1.44643500 & 1.33714500 \\
\hline $\mathrm{H}$ & 3.75150100 & 3.45330000 & 0.39208200 \\
\hline H & 4.83292000 & 1.35134400 & 1.86306100 \\
\hline
\end{tabular}

$\begin{array}{lll}2.97106100 & 0.41161400 & 1.29722700\end{array}$

$\begin{array}{llll}2.12849200 & 2.04552100 & 0.16204200\end{array}$

$\begin{array}{lll}0.21932600 & -0.36825500 & 0.16997000\end{array}$

$\begin{array}{lll}1.25289500 & 2.86089400 & -0.65712200\end{array}$

$\begin{array}{lll}1.30806000 & 2.71231700 & -2.05745300\end{array}$

$\begin{array}{llll}0.41553800 & 3.79765700 & -0.01687500\end{array}$

$\begin{array}{llll}0.46637900 & 3.53156300 & -2.82099000\end{array}$

$\begin{array}{lll}-0.39631300 & 4.59408600 & -0.83337900\end{array}$

$\begin{array}{lll}-0.37661700 & 4.46093600 & -2.21931300\end{array}$

$\begin{array}{lll}0.47994100 & 3.44495900 & -3.90325900\end{array}$

$\begin{array}{lll}-1.05109800 & 5.33037400 & -0.37796200\end{array}$

$\begin{array}{lll}-1.01614400 & 5.08932800 & -2.83273800\end{array}$

$\begin{array}{lll}3.18250000 & -0.88386700 & 1.91100300\end{array}$

$3.91809200 \quad-1.84910600 \quad 1.19337500$

$2.67221000 \quad-1.11254200 \quad 3.20418100$

$\begin{array}{lll}4.11565200 & -3.09107800 & 1.80946500\end{array}$

$\begin{array}{lll}2.90283800 & -2.37335000 & 3.76889400\end{array}$

$\begin{array}{lll}3.61111400 & -3.35404200 & 3.08018700\end{array}$

$\begin{array}{lll}4.67846300 & -3.86017000 & 1.28884100\end{array}$

$\begin{array}{lll}2.52703400 & -2.58707300 & 4.76487200\end{array}$

$3.77880800 \quad-4.32414100 \quad 3.53948500$

$\begin{array}{llll}0.39569400 & 3.99190900 & 1.49688400\end{array}$

$\begin{array}{llll}0.93310800 & 3.15522300 & 1.95604800\end{array}$

$\begin{array}{lll}1.12949500 & 5.28963600 & 1.89489200\end{array}$

$\begin{array}{lll}1.14059000 & 5.40622600 & 2.98454900\end{array}$

$\begin{array}{lll}2.16744600 & 5.29187400 & 1.54413500\end{array}$

$\begin{array}{lll}0.63364000 & 6.16829000 & 1.46707800\end{array}$

$\begin{array}{lll}-1.03350400 & 3.98090100 & 2.07182200\end{array}$

$\begin{array}{lll}-1.61423300 & 4.84880800 & 1.74082800\end{array}$

$\begin{array}{lll}-1.58011600 & 3.08111200 & 1.77013500\end{array}$

$\begin{array}{lll}-0.99595700 & 4.01395600 & 3.16683100\end{array}$

$\begin{array}{lll}1.92640500 & -0.04959400 & 4.00524200\end{array}$

$\begin{array}{llll}2.75946000 & 0.41163200 & 5.21877000\end{array}$

$\begin{array}{lll}3.73561100 & 0.80218600 & 4.91023700\end{array}$

$\begin{array}{lll}2.23394200 & 1.20397900 & 5.76398300\end{array}$

$\begin{array}{lll}2.93768300 & -0.41340400 & 5.91758200\end{array}$

$\begin{array}{lll}4.52206900 & -1.58243600 & -0.18321100\end{array}$

$\begin{array}{llll}4.20938500 & -0.58598200 & -0.51108200\end{array}$

$\begin{array}{llll}2.25156800 & 1.73940200 & -2.75896900\end{array}$

$\begin{array}{llll}3.29966300 & 2.49541800 & -3.60089200\end{array}$

$\begin{array}{llll}2.82879600 & 3.06476600 & -4.41011400\end{array}$

$\begin{array}{lll}3.87399900 & 3.19991000 & -2.98898400\end{array}$

$\begin{array}{lll}4.00285900 & 1.78900500 & -4.05637500\end{array}$

$\begin{array}{lll}4.01642300 & -2.58047700 & -1.24177000\end{array}$

$\begin{array}{llll}2.92598900 & -2.53867900 & -1.33490100\end{array}$

$\begin{array}{lll}4.44939000 & -2.34345300 & -2.22032200\end{array}$

$\begin{array}{lll}4.29845100 & -3.61057800 & -0.99666900\end{array}$

$6.06321700-1.57959100-0.11859600$

$\begin{array}{lll}6.48552300 & -1.33063500 & -1.09867000\end{array}$

$\begin{array}{lll}6.43375700 & -0.84666800 & 0.60676700\end{array}$

$\begin{array}{lll}6.45316600 & -2.56103800 & 0.17387800\end{array}$

$\begin{array}{lll}0.53081800 & -0.53291500 & 4.44337500\end{array}$

$\begin{array}{lll}-0.07152500 & -0.84288800 & 3.58220700\end{array}$

$\begin{array}{lll}0.59324000 & -1.38262100 & 5.13228800\end{array}$

$\begin{array}{lll}-0.00062700 & 0.27229200 & 4.96386300\end{array}$

$\begin{array}{lll}1.77746700 & 0.82359800 & 3.36198800\end{array}$

$\begin{array}{lll}1.48530100 & 0.71485600 & -3.61775700\end{array}$

$\begin{array}{llll}2.18672600 & 0.00845700 & -4.07638200\end{array}$

$\begin{array}{lll}0.77107000 & 0.14327700 & -3.01482500\end{array}$

$\begin{array}{lll}0.92975900 & 1.20114800 & -4.42751800\end{array}$

$\begin{array}{lll}2.79598700 & 1.17519100 & -1.99510300\end{array}$

$-1.27713200 \quad-2.45860200-1.57510200$

$-0.12922000 \quad-3.23610900-1.78913800$

$\begin{array}{lll}-2.31537000 & -2.53511600 & -2.53040300\end{array}$

$\begin{array}{lll}-0.02028300 & -4.08759300 & -2.88816900\end{array}$

$\begin{array}{llll}0.68116700 & -3.19428900 & -1.06757800\end{array}$

$\begin{array}{llll}-2.20812900 & -3.36138900 & -3.63850700\end{array}$ 


$\begin{array}{lrrr}\mathrm{H} & -3.19541300 & -1.90853600 & -2.41220700 \\ \mathrm{C} & -1.06162300 & -4.15402200 & -3.82516500 \\ \mathrm{H} & 0.87503900 & -4.68634400 & -3.00770600 \\ \mathrm{H} & -2.99284800 & -3.40521700 & -4.38746800 \\ \mathrm{O} & -1.06052300 & -4.93303400 & -4.93421200 \\ \mathrm{C} & 0.07734600 & -5.74594800 & -5.20005300 \\ \mathrm{H} & 0.24085900 & -6.47910800 & -4.40027900 \\ \mathrm{H} & -0.14324800 & -6.26912800 & -6.13122500 \\ \mathrm{H} & 0.98104500 & -5.13737800 & -5.32924900 \\ \mathrm{O} & -5.58998800 & -2.10513800 & -0.50943500 \\ \mathrm{O} & -4.91779100 & -3.60844800 & 1.00284100 \\ \mathrm{C} & -5.92456000 & -3.38464700 & -1.09402200 \\ \mathrm{H} & -6.93769500 & -3.31229400 & -1.48974600 \\ \mathrm{H} & -5.21749400 & -3.60165100 & -1.90188600 \\ \mathrm{C} & -5.77020500 & -4.34554900 & 0.09154200 \\ \mathrm{H} & -5.27756100 & -5.28433000 & -0.16775100 \\ \mathrm{H} & -6.71066500 & -4.55010700 & 0.61051300 \\ \mathrm{C} & -4.72920500 & -2.35428600 & 0.51403600 \\ \mathrm{H} & -3.51226700 & -2.30272400 & 0.02235800\end{array}$
Imaginary Frequency $=-808.9513$ $\begin{array}{llll}0.15946400 & 5.93888700 & 1.90337700\end{array}$ $\begin{array}{lll}-1.13652300 & 3.53553400 & 2.60336700\end{array}$ $\begin{array}{lll}-1.86658100 & 4.32418400 & 2.38935100\end{array}$ $\begin{array}{lll}-1.59367600 & 2.57441300 & 2.34664400\end{array}$ $\begin{array}{lll}-0.94605000 & 3.55149300 & 3.68288900\end{array}$ $\begin{array}{lll}2.39900800 & -0.22506500 & 3.88413200\end{array}$ $\begin{array}{lll}3.33075800 & 0.25240900 & 5.01611700\end{array}$ $\begin{array}{llll}4.22979900 & 0.73591600 & 4.61807600\end{array}$ $\begin{array}{llll}2.81274200 & 0.97463500 & 5.65748900\end{array}$ $\begin{array}{llll}3.65411200 & -0.58253100 & 5.64803700\end{array}$ $\begin{array}{lll}4.45684000 & -1.35830800 & -0.70565600\end{array}$ $\begin{array}{lll}3.90758400 & -0.44818500 & -0.96666700\end{array}$ $\begin{array}{llll}1.55376200 & 1.82296300 & -2.75020500\end{array}$ $\begin{array}{llll}2.44180700 & 2.69848000 & -3.65828900\end{array}$ $\begin{array}{llll}1.84049700 & 3.29377100 & -4.35493900\end{array}$ $\begin{array}{llll}3.05459200 & 3.39221000 & -3.07168900\end{array}$ $\begin{array}{llll}3.11521800 & 2.06985500 & -4.25198600\end{array}$ $\begin{array}{lll}4.02829300 & -2.44259300 & -1.71188800\end{array}$ $\begin{array}{llll}2.95859400 & -2.66209400 & -1.63403200\end{array}$ $\begin{array}{lll}4.23329200 & -2.10476700 & -2.73417400\end{array}$ $\begin{array}{lll}4.57981400 & -3.37803900 & -1.56223700\end{array}$ $\begin{array}{llll}5.96161800 & -1.04617600 & -0.84254100\end{array}$ $\begin{array}{llll}6.19737800 & -0.74136400 & -1.86852100\end{array}$ $\begin{array}{lll}6.26928000 & -0.23775300 & -0.16976300\end{array}$ $\begin{array}{lll}6.57164500 & -1.92520200 & -0.60475200\end{array}$ $\begin{array}{lll}1.11231800 & -0.85341300 & 4.45220900\end{array}$ $\begin{array}{lll}0.44301300 & -1.17297100 & 3.64587400\end{array}$ $\begin{array}{lll}1.32761700 & -1.72858400 & 5.07543200\end{array}$ $\begin{array}{lll}0.58102100 & -0.12638600 & 5.07774500\end{array}$ $\begin{array}{lll}2.09903700 & 0.65928300 & 3.31331800\end{array}$ $\begin{array}{lll}0.74749900 & 0.80388200 & -3.57752300\end{array}$ $\begin{array}{llll}1.42632500 & 0.17071800 & -4.16006700\end{array}$ $\begin{array}{lll}0.14773400 & 0.15512100 & -2.93005700\end{array}$ $\begin{array}{llll}0.07248400 & 1.30067800 & -4.28369600\end{array}$ $\begin{array}{lll}2.22080200 & 1.25049000 & -2.09904000\end{array}$ $\begin{array}{lll}-0.87771800 & -3.14466700 & -1.00081700\end{array}$ $\begin{array}{llll}0.34516700 & -3.80214300 & -0.75958000\end{array}$ $\begin{array}{lll}-1.67049000 & -3.64414100 & -2.06312400\end{array}$ $\begin{array}{llll}0.74147500 & -4.92229300 & -1.48765400\end{array}$ $\begin{array}{llll}0.98471900 & -3.44282600 & 0.04179600\end{array}$ $\begin{array}{lll}-1.27188900 & -4.73104200 & -2.82224100\end{array}$ $\begin{array}{lll}-2.59677100 & -3.13839900 & -2.31926100\end{array}$ $\begin{array}{lll}-0.06593100 & -5.39410700 & -2.53306000\end{array}$ $\begin{array}{lll}1.67686900 & -5.41117600 & -1.24106500\end{array}$ $\begin{array}{lll}-1.87128500 & -5.09229500 & -3.65220200\end{array}$ $\begin{array}{llll}0.22627300 & -6.45705300 & -3.32198500\end{array}$ $\begin{array}{lll}1.43412300 & -7.17147600 & -3.08733600\end{array}$ $\begin{array}{llll}1.45219100 & -7.60557400 & -2.07989800\end{array}$ $\begin{array}{lll}1.45377200 & -7.97250600 & -3.82742500\end{array}$ $\begin{array}{llll}2.31216300 & -6.52778600 & -3.22381600\end{array}$ $\begin{array}{lll}-5.09475200 & -1.67700600 & -1.32481600\end{array}$ $\begin{array}{lll}-6.82363500 & -0.77038500 & -0.29873100\end{array}$ $\begin{array}{llll}-6.22225500 & -1.96969700 & -2.20038600\end{array}$ $\begin{array}{lll}-5.92468200 & -1.71418900 & -3.21698800\end{array}$ $\begin{array}{lll}-6.42973200 & -3.03962200 & -2.12309900\end{array}$ $\begin{array}{llll}-7.33020200 & -1.08409600 & -1.62383400\end{array}$ $\begin{array}{lll}-8.28925100 & -1.58714400 & -1.50202500\end{array}$ $\begin{array}{lll}-7.46234600 & -0.14272000 & -2.16298600\end{array}$ $\begin{array}{llll}-5.52866400 & -1.05488900 & -0.24358300\end{array}$ $\begin{array}{lll}-3.29438500 & -2.64526000 & -0.36886000\end{array}$

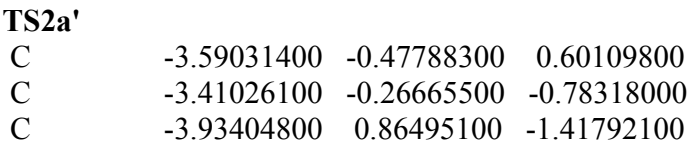




$\begin{array}{lrrr}\mathrm{H} & 2.43875700 & -2.73205800 & 3.97842000 \\ \mathrm{H} & 1.77065600 & -1.24668800 & 4.66490300 \\ \mathrm{H} & 2.63172500 & 0.08778500 & 2.79091900 \\ \mathrm{C} & -0.40602200 & 2.52700400 & -3.22699800 \\ \mathrm{H} & 0.16491400 & 2.29984200 & -4.13500700 \\ \mathrm{H} & -0.80233700 & 1.58780400 & -2.82721900 \\ \mathrm{H} & -1.25323100 & 3.15735700 & -3.52153700 \\ \mathrm{H} & 1.32471700 & 2.55607100 & -1.97769000 \\ \mathrm{C} & -1.26915400 & -3.40221000 & 0.51629300 \\ \mathrm{C} & 0.06748400 & -3.80599500 & 0.32757700 \\ \mathrm{C} & -2.21648600 & -4.42615700 & 0.77049600 \\ \mathrm{C} & 0.45911500 & -5.13909500 & 0.42471200 \\ \mathrm{H} & 0.82403600 & -3.05594100 & 0.11412200 \\ \mathrm{C} & -1.84538700 & -5.75648700 & 0.86322200 \\ \mathrm{H} & -3.26589200 & -4.16556600 & 0.87487600 \\ \mathrm{C} & -0.49847700 & -6.12805900 & 0.69956200 \\ \mathrm{H} & 1.50374200 & -5.39416500 & 0.28956100 \\ \mathrm{H} & -2.57679700 & -6.53524900 & 1.05468900 \\ \mathrm{O} & -0.23638000 & -7.45161200 & 0.80858300 \\ \mathrm{C} & 1.10588500 & -7.90159900 & 0.66170100 \\ \mathrm{H} & 1.76070600 & -7.45746800 & 1.42162300 \\ \mathrm{H} & 1.07252300 & -8.98298700 & 0.79895300 \\ \mathrm{H} & 1.49666600 & -7.67286300 & -0.33776200 \\ \mathrm{O} & -3.27588500 & -2.50010200 & -1.74084700 \\ \mathrm{O} & -1.87454000 & -0.96152400 & -2.51356400 \\ \mathrm{C} & -2.48557500 & -3.19535200 & -2.73404200 \\ \mathrm{H} & -1.76494500 & -3.83817700 & -2.22340700 \\ \mathrm{H} & -3.17158200 & -3.79121700 & -3.33601100 \\ \mathrm{C} & -1.82654000 & -2.03797300 & -3.48599800 \\ \mathrm{H} & -2.38731800 & -1.71597800 & -4.36785600 \\ \mathrm{H} & -0.78283600 & -2.21431000 & -3.74800700 \\ \mathrm{C} & -2.67239000 & -1.31934700 & -1.49270700 \\ \mathrm{H} & -3.15606100 & -2.09276400 & 2.01214700 \\ \mathrm{I} & & \end{array}$
Imaginary Frequency $=-231.9524$

$\begin{array}{crrr}\text { Int3a' } & & & \\ \mathrm{C} & -3.35018000 & -0.12678300 & -0.45791100 \\ \mathrm{C} & -3.19625300 & -0.69041100 & -1.73280100 \\ \mathrm{C} & -3.80219900 & -0.12015500 & -2.84015700 \\ \mathrm{C} & -4.57940600 & 1.03421500 & -2.65527600 \\ \mathrm{C} & -4.73864700 & 1.59234100 & -1.38391500 \\ \mathrm{C} & -4.11979300 & 1.01759100 & -0.26659300 \\ \mathrm{C} & -2.58322400 & -0.93688100 & 0.51332000 \\ \mathrm{C} & -1.95071200 & -2.00042800 & -0.12369000 \\ \mathrm{H} & -3.70009900 & -0.55925900 & -3.82819400 \\ \mathrm{H} & -5.07361300 & 1.49000500 & -3.50819100 \\ \mathrm{H} & -5.35217000 & 2.47980900 & -1.25975700 \\ \mathrm{H} & -4.23930700 & 1.45610200 & 0.72017400 \\ \mathrm{C} & 1.31360300 & 0.89279600 & 0.44648600 \\ \mathrm{C} & 2.67547700 & 2.66570500 & 0.74021900 \\ \mathrm{C} & 3.46008200 & 1.57426600 & 0.54640100 \\ \mathrm{H} & 2.92235300 & 3.70052300 & 0.91756000 \\ \mathrm{H} & 4.53228700 & 1.46142500 & 0.51885500 \\ \mathrm{~N} & 2.60839400 & 0.49237100 & 0.36785500 \\ \mathrm{~N} & 1.35914200 & 2.23083200 & 0.67555300 \\ \mathrm{Au} & -0.36793400 & -0.24203300 & 0.29740900 \\ \mathrm{C} & 0.20951800 & 3.09817900 & 0.84699600 \\ \mathrm{C} & -0.33279100 & 3.72575700 & -0.29180500 \\ \mathrm{C} & -0.29272300 & 3.29335400 & 2.14959400 \\ \mathrm{C} & -1.43172800 & 4.57041200 & -0.09059000 \\ \mathrm{C} & -1.39387800 & 4.14760500 & 2.28672900 \\ \mathrm{C} & -1.95866600 & 4.77872500 & 1.18118800 \\ \mathrm{H} & -1.87660200 & 5.07489900 & -0.94289300 \\ \mathrm{H} & -1.80892700 & 4.32619900 & 3.27420800 \\ \mathrm{H} & -2.80841400 & 5.44270500 & 1.31302700\end{array}$




\begin{tabular}{|c|c|c|c|}
\hline $\mathrm{C}$ & 3.06871800 & -0.86260200 & 0.13523100 \\
\hline $\mathrm{C}$ & 3.33186000 & -1.26070600 & -1.19084200 \\
\hline $\mathrm{C}$ & 3.27146500 & -1.70160400 & 1.24894700 \\
\hline $\mathrm{C}$ & 3.81568000 & -2.56126400 & -1.38108500 \\
\hline $\mathrm{C}$ & 3.75557100 & -2.99106100 & 0.99501700 \\
\hline $\mathrm{C}$ & 4.02714100 & -3.41669900 & -0.30259400 \\
\hline $\mathrm{H}$ & 4.04030800 & -2.90306300 & -2.38694300 \\
\hline $\mathrm{H}$ & 3.93082900 & -3.66523900 & 1.82794500 \\
\hline $\mathrm{H}$ & 4.41552900 & -4.41693800 & -0.47443700 \\
\hline $\mathrm{C}$ & 0.31238900 & 2.63198600 & 3.38499700 \\
\hline $\mathrm{H}$ & 1.16211600 & 2.01921700 & 3.06637800 \\
\hline $\mathrm{C}$ & 0.85313400 & 3.68079800 & 4.37759500 \\
\hline $\mathrm{H}$ & 1.33344200 & 3.18577100 & 5.22891800 \\
\hline $\mathrm{H}$ & 1.59200400 & 4.33710700 & 3.90477900 \\
\hline $\mathrm{H}$ & 0.04991800 & 4.31330500 & 4.77142400 \\
\hline $\mathrm{C}$ & -0.69490300 & 1.68881500 & 4.07191400 \\
\hline $\mathrm{H}$ & -1.57282100 & 2.23391900 & 4.43632100 \\
\hline $\mathrm{H}$ & -1.04290700 & 0.91066000 & 3.38261700 \\
\hline $\mathrm{H}$ & -0.22875000 & 1.19658600 & 4.93297400 \\
\hline $\mathrm{C}$ & 2.99693700 & -1.26310600 & 2.68445700 \\
\hline $\mathrm{C}$ & 4.27898400 & -1.29520600 & 3.54038900 \\
\hline $\mathrm{H}$ & 5.06723200 & -0.66904800 & 3.10798800 \\
\hline $\mathrm{H}$ & 4.06864900 & -0.92707000 & 4.55072200 \\
\hline $\mathrm{H}$ & 4.67560500 & -2.31224300 & 3.63448300 \\
\hline $\mathrm{C}$ & 3.14152300 & -0.33630100 & -2.39024300 \\
\hline $\mathrm{H}$ & 2.67914900 & 0.59144800 & -2.03769200 \\
\hline $\mathrm{C}$ & 0.22489200 & 3.52928800 & -1.69853400 \\
\hline $\mathrm{C}$ & 0.74800100 & 4.85562000 & -2.28575200 \\
\hline $\mathrm{H}$ & -0.05728500 & 5.58943100 & -2.40185200 \\
\hline $\mathrm{H}$ & 1.51623300 & 5.30364100 & -1.64571000 \\
\hline $\mathrm{H}$ & 1.18697400 & 4.68508800 & -3.27509000 \\
\hline $\mathrm{C}$ & 2.18781900 & -0.94082800 & -3.43837300 \\
\hline $\mathrm{H}$ & 1.21185500 & -1.17598300 & -3.00128500 \\
\hline $\mathrm{H}$ & 2.03302700 & -0.22845200 & -4.25681100 \\
\hline $\mathrm{H}$ & 2.59906500 & -1.85749200 & -3.87722500 \\
\hline $\mathrm{C}$ & 4.49846800 & 0.03649400 & -3.02152100 \\
\hline $\mathrm{H}$ & 4.35227400 & 0.73392400 & -3.85385000 \\
\hline $\mathrm{H}$ & 5.16506000 & 0.51191000 & -2.29321200 \\
\hline $\mathrm{H}$ & 5.01269400 & -0.84833300 & -3.41330000 \\
\hline $\mathrm{C}$ & 1.87881300 & -2.10792500 & 3.32656000 \\
\hline $\mathrm{H}$ & 0.95527400 & -2.06139200 & 2.73895100 \\
\hline $\mathrm{H}$ & 2.16818500 & -3.16149100 & 3.40935000 \\
\hline $\mathrm{H}$ & 1.66249400 & -1.74313200 & 4.33719900 \\
\hline $\mathrm{H}$ & 2.64732900 & -0.22560100 & 2.66590300 \\
\hline $\mathrm{C}$ & -0.81601700 & 2.87978000 & -2.63117500 \\
\hline $\mathrm{H}$ & -0.38046100 & 2.71042000 & -3.62258600 \\
\hline $\mathrm{H}$ & -1.15894600 & 1.91661200 & -2.23839300 \\
\hline $\mathrm{H}$ & -1.69562800 & 3.52023300 & -2.75962000 \\
\hline $\mathrm{H}$ & 1.07656100 & 2.84366000 & -1.63890000 \\
\hline $\mathrm{C}$ & -1.38321500 & -3.20111700 & 0.50956800 \\
\hline $\mathrm{C}$ & -0.31477300 & -3.90287300 & -0.07563300 \\
\hline $\mathrm{C}$ & -1.90737500 & -3.68849400 & 1.72679500 \\
\hline $\mathrm{C}$ & 0.21404000 & -5.04765600 & 0.51674600 \\
\hline $\mathrm{H}$ & 0.12792800 & -3.52836500 & -0.99224100 \\
\hline $\mathrm{C}$ & -1.39168000 & -4.82607000 & 2.32331200 \\
\hline $\mathrm{H}$ & -2.75347200 & -3.18762100 & 2.18823500 \\
\hline $\mathrm{C}$ & -0.32527900 & -5.52081000 & 1.72284700 \\
\hline $\mathrm{H}$ & 1.04501300 & -5.55458800 & 0.04091500 \\
\hline $\mathrm{H}$ & -1.80734100 & -5.21522600 & 3.24701400 \\
\hline $\mathrm{O}$ & 0.10029600 & -6.62310500 & 2.38070400 \\
\hline $\mathrm{C}$ & 1.13894900 & -7.41064400 & 1.80734600 \\
\hline $\mathrm{H}$ & 2.07110800 & -6.83857400 & 1.71834400 \\
\hline $\mathrm{H}$ & 1.29017500 & -8.24511400 & 2.49278700 \\
\hline $\mathrm{H}$ & 0.84908500 & -7.79494200 & 0.82179200 \\
\hline $\mathrm{O}$ & -3.16100800 & -3.08465000 & -1.94192600 \\
\hline $\mathrm{O}$ & -1.22777600 & -2.00584200 & -2.49960600 \\
\hline $\mathrm{C}$ & 2434003 & -3.945594 & -2825 \\
\hline
\end{tabular}

$\begin{array}{lrrr}\mathrm{H} & -1.88436700 & -4.70609300 & -2.25937200 \\ \mathrm{H} & -3.15534000 & -4.42925800 & -3.48828200 \\ \mathrm{C} & -1.49751800 & -2.97040400 & -3.53016700 \\ \mathrm{H} & -1.97615500 & -2.48658700 & -4.39066900 \\ \mathrm{H} & -0.54486500 & -3.40464600 & -3.84134400 \\ \mathrm{C} & -2.35354100 & -1.95974100 & -1.63825600 \\ \mathrm{H} & -2.71057900 & -0.85154300 & 1.58936400\end{array}$

\begin{tabular}{|c|c|c|c|}
\hline Int1b & & & \\
\hline $\mathrm{C}$ & -3.22125200 & 0.22224300 & -0.22510400 \\
\hline $\mathrm{C}$ & -4.35218200 & -0.36464900 & 0.39328500 \\
\hline $\mathrm{C}$ & -5.37235000 & 0.46785800 & 0.85104800 \\
\hline $\mathrm{C}$ & -5.28630400 & 1.85536900 & 0.70929400 \\
\hline $\mathrm{C}$ & -4.16927900 & 2.43109400 & 0.10351900 \\
\hline $\mathrm{C}$ & -3.13639300 & 1.61870500 & -0.35815000 \\
\hline $\mathrm{C}$ & -2.16871000 & -0.62576400 & -0.72785100 \\
\hline $\mathrm{C}$ & -1.57078100 & -1.57180000 & -1.26216400 \\
\hline $\mathrm{H}$ & -6.24322100 & 0.01316500 & 1.30820900 \\
\hline $\mathrm{H}$ & -6.09573800 & 2.48348800 & 1.06967300 \\
\hline $\mathrm{H}$ & -4.10008500 & 3.50833900 & -0.01274600 \\
\hline $\mathrm{H}$ & -2.26096800 & 2.05704600 & -0.82770400 \\
\hline $\mathrm{C}$ & 1.72565700 & 0.68275700 & 0.47626200 \\
\hline $\mathrm{C}$ & 3.33327900 & 2.18054700 & 0.97510300 \\
\hline $\mathrm{C}$ & 3.75318500 & 0.96580700 & 1.41553100 \\
\hline $\mathrm{H}$ & 3.79623500 & 3.15376900 & 1.01580200 \\
\hline $\mathrm{H}$ & 4.65862200 & 0.66311100 & 1.91722700 \\
\hline $\mathrm{N}$ & 2.75388000 & 0.05519400 & 1.10173900 \\
\hline $\mathrm{N}$ & 2.08417900 & 1.99018000 & 0.40106200 \\
\hline $\mathrm{Au}$ & 0.00877300 & -0.14110000 & -0.20958800 \\
\hline $\mathrm{C}$ & 1.27337800 & 3.05162900 & -0.16338700 \\
\hline $\mathrm{C}$ & 1.34796100 & 3.29274700 & -1.55005100 \\
\hline $\mathrm{C}$ & 0.46361300 & 3.80688400 & 0.70928400 \\
\hline $\mathrm{C}$ & 0.55661900 & 4.32996400 & -2.05993600 \\
\hline $\mathrm{C}$ & -0.30151600 & 4.83326400 & 0.14076500 \\
\hline $\mathrm{C}$ & -0.25918100 & 5.09182300 & -1.22717800 \\
\hline $\mathrm{H}$ & 0.58696400 & 4.54833400 & -3.12314200 \\
\hline $\mathrm{H}$ & -0.93482500 & 5.44018100 & 0.78076700 \\
\hline $\mathrm{H}$ & -0.85848900 & 5.89564200 & -1.64540300 \\
\hline $\mathrm{C}$ & 2.82056000 & -1.36105700 & 1.40764100 \\
\hline $\mathrm{C}$ & 3.49194100 & -2.20869400 & 0.50365200 \\
\hline $\mathrm{C}$ & 2.23922900 & -1.81720300 & 2.60784000 \\
\hline $\mathrm{C}$ & 3.56843000 & -3.56595600 & 0.83957900 \\
\hline $\mathrm{C}$ & 2.34553000 & -3.18560200 & 2.88644100 \\
\hline $\mathrm{C}$ & 3.00204300 & -4.05083500 & 2.01540200 \\
\hline $\mathrm{H}$ & 4.08149900 & -4.25121600 & 0.17171100 \\
\hline $\mathrm{H}$ & 1.91397100 & -3.57602600 & 3.80302800 \\
\hline $\mathrm{H}$ & 3.07722700 & -5.10741500 & 2.25654300 \\
\hline $\mathrm{C}$ & 0.40595600 & 3.56302600 & 2.21520100 \\
\hline $\mathrm{H}$ & 1.02578300 & 2.69089400 & 2.44736600 \\
\hline $\mathrm{C}$ & 0.98973200 & 4.75877500 & 2.99511700 \\
\hline $\mathrm{H}$ & 0.98965700 & 4.54781000 & 4.07031500 \\
\hline $\mathrm{H}$ & 2.02036500 & 4.97393100 & 2.69149800 \\
\hline $\mathrm{H}$ & 0.39974600 & 5.66795100 & 2.83404100 \\
\hline $\mathrm{C}$ & -1.02382000 & 3.23835200 & 2.68921600 \\
\hline $\mathrm{H}$ & -1.70365200 & 4.08290200 & 2.53041600 \\
\hline $\mathrm{H}$ & -1.43531600 & 2.37345400 & 2.15819100 \\
\hline $\mathrm{H}$ & -1.02251700 & 3.01443800 & 3.76189500 \\
\hline $\mathrm{C}$ & 1.53528600 & -0.89530100 & 3.59942500 \\
\hline $\mathrm{C}$ & 2.30429900 & -0.82368500 & 4.93449200 \\
\hline $\mathrm{H}$ & 3.33589900 & -0.48553100 & 4.78619600 \\
\hline $\mathrm{H}$ & 1.81244600 & -0.12424300 & 5.61974900 \\
\hline $\mathrm{H}$ & 2.34291000 & -1.80150500 & 5.42733300 \\
\hline $\mathrm{C}$ & 4.13842300 & -1.70872600 & -0.78517200 \\
\hline $\mathrm{H}$ & 3.89510600 & -0.64762000 & -0.90404500 \\
\hline $\mathrm{C}$ & 2.25181700 & 2.49822500 & -2.48816700 \\
\hline
\end{tabular}




\begin{tabular}{lrrr}
$\mathrm{C}$ & 3.34697100 & 3.39524400 & -3.10005000 \\
$\mathrm{H}$ & 2.91582600 & 4.18368300 & -3.72686300 \\
$\mathrm{H}$ & 3.94923500 & 3.87959300 & -2.32355700 \\
$\mathrm{H}$ & 4.01831500 & 2.79986800 & -3.72893100 \\
$\mathrm{C}$ & 3.58352900 & -2.43778400 & -2.02374900 \\
$\mathrm{H}$ & 2.49393500 & -2.34592700 & -2.08628400 \\
$\mathrm{H}$ & 4.01823400 & -2.01318600 & -2.93589900 \\
$\mathrm{H}$ & 3.82846700 & -3.50547200 & -2.00895500 \\
$\mathrm{C}$ & 5.67494000 & -1.82347900 & -0.71747900 \\
$\mathrm{H}$ & 6.12807500 & -1.42226200 & -1.63092700 \\
$\mathrm{H}$ & 6.08367000 & -1.27015600 & 0.13546200 \\
$\mathrm{H}$ & 5.99224300 & -2.86756000 & -0.61779300 \\
$\mathrm{C}$ & 0.06871500 & -1.31228700 & 3.82552100 \\
$\mathrm{H}$ & -0.49270200 & -1.33373300 & 2.88449200 \\
$\mathrm{H}$ & -0.00093300 & -2.30602700 & 4.28218700 \\
$\mathrm{H}$ & -0.42332800 & -0.60341800 & 4.50128000 \\
$\mathrm{H}$ & 1.51916800 & 0.11554500 & 3.17929600 \\
$\mathrm{C}$ & 1.44266100 & 1.78372700 & -3.58826600 \\
$\mathrm{H}$ & 2.10969200 & 1.18503400 & -4.21885200 \\
$\mathrm{H}$ & 0.68822100 & 1.11666600 & -3.15476000 \\
$\mathrm{H}$ & 0.92519700 & 2.49784400 & -4.23852400 \\
$\mathrm{H}$ & 2.75634100 & 1.72128800 & -1.90467900 \\
$\mathrm{C}$ & -1.07037500 & -2.76750100 & -1.87107400 \\
$\mathrm{C}$ & -0.44110600 & -2.74150500 & -3.13078700 \\
$\mathrm{C}$ & -1.26276100 & -3.99397500 & -1.20249800 \\
$\mathrm{C}$ & -0.01329300 & -3.93199700 & -3.71198600 \\
$\mathrm{H}$ & -0.30280000 & -1.79428600 & -3.64292400 \\
$\mathrm{C}$ & -0.83414300 & -5.17642300 & -1.79873700 \\
$\mathrm{H}$ & -1.75949100 & -3.99609000 & -0.23699400 \\
$\mathrm{C}$ & -0.20901100 & -5.14778500 & -3.04918700 \\
$\mathrm{H}$ & 0.46628900 & -3.91374700 & -4.68597300 \\
$\mathrm{H}$ & -0.98924500 & -6.12284800 & -1.28935000 \\
$\mathrm{O}$ & -5.70032400 & -2.23816400 & 1.07572400 \\
$\mathrm{O}$ & -3.43291900 & -2.41859200 & 1.37827700 \\
$\mathrm{C}$ & -5.46644400 & -3.28059000 & 2.02525700 \\
$\mathrm{H}$ & -6.26162100 & -3.24244500 & 2.77283400 \\
$\mathrm{H}$ & -5.47132300 & -4.26263900 & 1.53233700 \\
$\mathrm{C}$ & -4.08217000 & -2.92180200 & 2.55189200 \\
$\mathrm{H}$ & -3.50663900 & -3.77430400 & 2.92050300 \\
$\mathrm{H}$ & -4.13446600 & -2.14967900 & 3.33117300 \\
$\mathrm{C}$ & -4.45516200 & -1.87828700 & 0.52479900 \\
$\mathrm{H}$ & -4.31865800 & -2.35279500 & -0.45854900 \\
$\mathrm{H}$ & 0.12231700 & -6.07375900 & -3.51001100 \\
& & & \\
\hline
\end{tabular}

$\begin{array}{lrrr}\text { TS1b } & & & \\ \mathrm{C} & -3.26585800 & 0.07438500 & -0.13356300 \\ \mathrm{C} & -4.59255500 & -0.37422700 & -0.31325200 \\ \mathrm{C} & -5.66561000 & 0.51290400 & -0.22556700 \\ \mathrm{C} & -5.43742200 & 1.85239700 & 0.08895800 \\ \mathrm{C} & -4.13104800 & 2.30405500 & 0.29011000 \\ \mathrm{C} & -3.05442800 & 1.42551900 & 0.17616000 \\ \mathrm{C} & -2.15660100 & -0.87758900 & -0.33353700 \\ \mathrm{C} & -2.33494600 & -2.07330300 & -0.82693900 \\ \mathrm{H} & -6.67407100 & 0.14764300 & -0.39286500 \\ \mathrm{H} & -6.27486100 & 2.53788700 & 0.17656100 \\ \mathrm{H} & -3.94732700 & 3.34820500 & 0.52545100 \\ \mathrm{H} & -2.04006300 & 1.78742900 & 0.31061200 \\ \mathrm{C} & 1.77214100 & 0.14360900 & 0.54521000 \\ \mathrm{C} & 3.60671600 & 1.41762600 & 0.90107700 \\ \mathrm{C} & 3.96341900 & 0.12332100 & 1.10241700 \\ \mathrm{H} & 4.17443600 & 2.33244300 & 0.96493500 \\ \mathrm{H} & 4.90574500 & -0.32241800 & 1.37914600 \\ \mathrm{~N} & 2.82709500 & -0.64551200 & 0.88154300 \\ \mathrm{~N} & 2.26016200 & 1.41316900 & 0.56169500 \\ \mathrm{Au} & -0.15088000 & -0.40680100 & 0.09020100\end{array}$

$\begin{array}{lll}1.48683800 & 2.60624500 & 0.28358800\end{array}$ $\begin{array}{llll}1.40903100 & 3.06350100 & -1.04696600\end{array}$ $\begin{array}{lll}0.86260300 & 3.26604400 & 1.36189700\end{array}$ $\begin{array}{llll}0.65663300 & 4.22113200 & -1.28272900\end{array}$ $\begin{array}{llll}0.12558400 & 4.41967600 & 1.06449900\end{array}$ $\begin{array}{llll}0.02058100 & 4.89207100 & -0.24164200\end{array}$ $\begin{array}{llll}0.57259900 & 4.60345500 & -2.29549900\end{array}$ $\begin{array}{lll}-0.36587100 & 4.95910100 & 1.86881200\end{array}$ $\begin{array}{lll}-0.55276700 & 5.79162800 & -0.44775500\end{array}$ $\begin{array}{llll}2.79610400 & -2.08707000 & 1.01898000\end{array}$ $3.11066700-2.87727500 \quad-0.10444700$ $\begin{array}{llll}2.49499300 & -2.63294200 & 2.28330100\end{array}$ $\begin{array}{llll}3.11353900 & -4.26679500 & 0.07124900\end{array}$ $\begin{array}{lll}2.51212000 & -4.02894000 & 2.39784500\end{array}$ $\begin{array}{lll}2.82050000 & -4.83787400 & 1.30692200\end{array}$ $\begin{array}{lll}3.35640300 & -4.90839100 & -0.77053100\end{array}$ $\begin{array}{llll}2.29247300 & -4.48647700 & 3.35808500\end{array}$ $\begin{array}{lll}2.84264600 & -5.91815700 & 1.42316400\end{array}$ $\begin{array}{lll}0.97262100 & 2.78790200 & 2.80710600\end{array}$ $\begin{array}{lll}1.56366800 & 1.86659200 & 2.81998500\end{array}$ $\begin{array}{lll}1.71484800 & 3.81813700 & 3.68210100\end{array}$ $\begin{array}{llll}1.83190800 & 3.43590100 & 4.70237600\end{array}$ $\begin{array}{lll}2.71216400 & 4.03771600 & 3.28505300\end{array}$ $\begin{array}{lll}1.16549200 & 4.76434200 & 3.74255900\end{array}$ $\begin{array}{lll}-0.40692100 & 2.44598000 & 3.40283400\end{array}$ $\begin{array}{lll}-1.05350300 & 3.32912900 & 3.45900100\end{array}$ $\begin{array}{lll}-0.92254100 & 1.68676900 & 2.80416600\end{array}$ $\begin{array}{lll}-0.29261100 & 2.05692200 & 4.42098000\end{array}$ $\begin{array}{lll}2.17273400 & -1.77713500 & 3.50505400\end{array}$ $\begin{array}{llll}3.21087000 & -1.98286700 & 4.62632600\end{array}$ $\begin{array}{lll}4.22678200 & -1.76672300 & 4.27753900\end{array}$ $\begin{array}{lll}2.99362200 & -1.31957700 & 5.47104600\end{array}$ $\begin{array}{lll}3.19991500 & -3.01223300 & 5.00155000\end{array}$ $3.45259500 \quad-2.28454100 \quad-1.46836000$ $3.38199100 \quad-1.19472600 \quad-1.39537700$ $\begin{array}{lll}2.10569900 & 2.36238500 & -2.20914000\end{array}$ $\begin{array}{lll}3.15139000 & 3.28021800 & -2.87347700\end{array}$ $\begin{array}{lll}2.68349500 & 4.16029700 & -3.32871700\end{array}$ $3.89273600 \quad 3.63482400 \quad-2.14877500$ $\begin{array}{lll}3.68204200 & 2.73998600 & -3.66556200\end{array}$ $\begin{array}{lll}2.44683700 & -2.73175100 & -2.54716200\end{array}$ $\begin{array}{lll}1.42291500 & -2.45271500 & -2.27509400\end{array}$ $\begin{array}{llll}2.68711000 & -2.25965700 & -3.50660100\end{array}$ $\begin{array}{lll}2.47189800 & -3.81724000 & -2.69613600\end{array}$ $\begin{array}{llll}4.89884600 & -2.62291300 & -1.88169100\end{array}$ $\begin{array}{lll}5.14099000 & -2.14702100 & -2.83852000\end{array}$ $\begin{array}{lll}5.62203300 & -2.27401900 & -1.13614500\end{array}$ $\begin{array}{lll}5.04044900 & -3.70280600 & -2.00232900\end{array}$ $\begin{array}{lll}0.74268700 & -2.03867600 & 4.01766900\end{array}$ $\begin{array}{lll}-0.00083100 & -1.83685900 & 3.23803500\end{array}$ $\begin{array}{lll}0.61638500 & -3.07595500 & 4.34851000\end{array}$ $\begin{array}{llll}0.52180200 & -1.38988400 & 4.87282700\end{array}$ $\begin{array}{lll}2.21927800 & -0.72436300 & 3.20944000\end{array}$ $\begin{array}{llr}1.08906000 & 1.83809500 & -3.24259800\end{array}$ $\begin{array}{llll}1.60697900 & 1.29851400 & -4.04373200\end{array}$ $\begin{array}{lll}0.36945800 & 1.15402600 & -2.77844300\end{array}$ $\begin{array}{llll}0.52641700 & 2.65750400 & -3.70418900\end{array}$ $\begin{array}{lll}2.64189500 & 1.49356400 & -1.81391800\end{array}$ $\begin{array}{lll}-1.86994800 & -3.42405300 & -1.00858200\end{array}$ $-2.04528600 \quad-4.07913400 \quad-2.24634600$ $\begin{array}{lll}-1.24663900 & -4.11056700 & 0.05577300\end{array}$ $\begin{array}{lll}-1.60176900 & -5.38653400 & -2.41533100\end{array}$ $\begin{array}{lll}-2.52540000 & -3.55011100 & -3.06469900\end{array}$ $-0.79247500 \quad-5.41322800 \quad-0.12789400$ $\begin{array}{lll}-1.11481500 & -3.60568800 & 1.00694700\end{array}$ $\begin{array}{lll}-0.97397700 & -6.05386400 & -1.35745400\end{array}$ $\begin{array}{lll}-1.73726900 & -5.88511900 & -3.37039700\end{array}$ 


$\begin{array}{lrrr}\mathrm{H} & -0.30027300 & -5.93023200 & 0.68985300 \\ \mathrm{O} & -5.78944500 & -2.06085800 & -1.59295100 \\ \mathrm{O} & -5.03229900 & -2.63937000 & 0.44467700 \\ \mathrm{C} & -6.50336600 & -3.26593400 & -1.23099200 \\ \mathrm{H} & -7.51920600 & -2.98280100 & -0.93918800 \\ \mathrm{H} & -6.54050800 & -3.92724200 & -2.09895600 \\ \mathrm{C} & -5.68065500 & -3.82024000 & -0.06386900 \\ \mathrm{H} & -4.92586500 & -4.54438000 & -0.39210200 \\ \mathrm{H} & -6.27674000 & -4.24621100 & 0.74387300 \\ \mathrm{C} & -4.83452700 & -1.80999800 & -0.63698500 \\ \mathrm{H} & -3.73567800 & -2.12827800 & -1.14017000 \\ \mathrm{H} & -0.62687700 & -7.07425900 & -1.49289700 \\ \mathrm{Imaginary} & \text { Frequency }=-581.8634 & \end{array}$
Imaginary Frequency $\begin{array}{lll}3.28777300 & -0.44595600 & 4.80920400\end{array}$ $\begin{array}{lll}1.76510900 & -0.06640300 & 5.63610100\end{array}$ $\begin{array}{llll}2.28833600 & -1.74862700 & 5.46718000\end{array}$ $\begin{array}{lll}4.11916400 & -1.74660000 & -0.72710500\end{array}$ $\begin{array}{lll}3.88646900 & -0.68460800 & -0.85889800\end{array}$ $\begin{array}{llll}2.26899200 & 2.43514500 & -2.50591800\end{array}$ $3.37844600 \quad 3.31354200 \quad-3.11905100$ $\begin{array}{llll}2.96040700 & 4.09823200 & -3.75931600\end{array}$ $\begin{array}{llll}3.97855200 & 3.80175200 & -2.34331200\end{array}$ $\begin{array}{lll}4.04947200 & 2.70385200 & -3.73441700\end{array}$ $\begin{array}{lll}3.57054000 & -2.48295600 & -1.96405900\end{array}$ $2.48264600 \quad-2.38082000 \quad-2.03919800$ $\begin{array}{lll}4.01884600 & -2.07202800 & -2.87587300\end{array}$ $\begin{array}{lll}3.80464400 & -3.55276900 & -1.93585400\end{array}$ $\begin{array}{llll}5.65386000 & -1.87403400 & -0.64290900\end{array}$ $6.11950400 \quad-1.48545100-1.55553100$ $\begin{array}{lll}6.05891000 & -1.31624800 & 0.20886400\end{array}$ $\begin{array}{lll}5.96089700 & -2.91984500 & -0.53020600\end{array}$ $\begin{array}{lll}0.01781100 & -1.27095000 & 3.85731600\end{array}$ $\begin{array}{lll}-0.54335200 & -1.30705800 & 2.91658700\end{array}$ $\begin{array}{lll}-0.05660300 & -2.25591800 & 4.33191600\end{array}$ $\begin{array}{lll}-0.47162000 & -0.54808500 & 4.52005700\end{array}$ $\begin{array}{lll}1.47594600 & 0.14042400 & 3.19167100\end{array}$ $\begin{array}{lll}1.46414700 & 1.71306400 & -3.60425200\end{array}$ $\begin{array}{llll}2.13149900 & 1.09831000 & -4.21879000\end{array}$ $\begin{array}{lll}0.69824400 & 1.06018200 & -3.16917000\end{array}$ $\begin{array}{lll}0.96096200 & 2.42265200 & -4.27046400\end{array}$ $\begin{array}{lll}2.75999400 & 1.66138200 & -1.90692300\end{array}$ $\begin{array}{lll}-1.07282100 & -2.78577600 & -1.83341900\end{array}$ $-0.44803400 \quad-2.77871900-3.09511500$ $\begin{array}{lll}-1.25407300 & -4.00151600 & -1.14212400\end{array}$ $-0.01458200 \quad-3.97378000 \quad-3.65929200$ $\begin{array}{lll}-0.31690500 & -1.84088800 & -3.62573600\end{array}$ $\begin{array}{lll}-0.81746200 & -5.18852100 & -1.71559000\end{array}$ $\begin{array}{lll}-1.74546300 & -3.99129200 & -0.17411700\end{array}$ $\begin{array}{lll}-0.19613900 & -5.18271500 & -2.97239400\end{array}$ $\begin{array}{llll}0.45980100 & -3.97722700 & -4.63371100\end{array}$ $\begin{array}{llll}-0.94931800 & -6.13736200 & -1.20662400\end{array}$ $\begin{array}{lll}-5.65675300 & -2.22562700 & 1.23176200\end{array}$ $\begin{array}{lll}-3.38021500 & -2.38469500 & 1.46746400\end{array}$ $\begin{array}{lll}-5.38745800 & -3.27222900 & 2.16744400\end{array}$ $\begin{array}{lll}-6.16076100 & -3.24435900 & 2.93785900\end{array}$ $\begin{array}{lll}-5.39919900 & -4.25113400 & 1.66849000\end{array}$ $\begin{array}{lll}-3.99097700 & -2.90486800 & 2.65506400\end{array}$ $\begin{array}{lll}-3.39794700 & -3.75555400 & 2.99947000\end{array}$ $\begin{array}{lll}-4.02657300 & -2.13963600 & 3.44182000\end{array}$ $\begin{array}{lll}-4.43340600 & -1.87398100 & 0.63247200\end{array}$ $\begin{array}{lll}-4.32490400 & -2.37297100 & -0.34266300\end{array}$ $\begin{array}{lll}0.25062900 & -6.49853700 & -3.53009800\end{array}$ $\begin{array}{lll}0.12557000 & -7.55424600 & -2.94341600\end{array}$ $\begin{array}{lll}0.80404400 & -6.37520100 & -4.75039600\end{array}$ $\begin{array}{lll}1.24568800 & -7.60556000 & -5.35687000\end{array}$ $\begin{array}{lll}0.40467100 & -8.29304000 & -5.47411300\end{array}$ $\begin{array}{lll}1.65121100 & -7.32131400 & -6.32748300\end{array}$ $\begin{array}{lll}2.01270600 & -8.07923300 & -4.73928600\end{array}$

$\begin{array}{lrrr}\text { TS1c } & & & \\ \text { C } & -3.29984600 & 0.14230600 & -0.19720700 \\ \text { C } & -4.62803900 & -0.29496400 & -0.39249400 \\ \text { C } & -5.68627500 & 0.61356600 & -0.37897200 \\ \text { C } & -5.44272200 & 1.96356300 & -0.12546600 \\ \text { C } & -4.13523800 & 2.40361100 & 0.09273200 \\ \text { C } & -3.07181600 & 1.50272700 & 0.05343900 \\ \text { C } & -2.20442000 & -0.83653700 & -0.31417300 \\ \text { C } & -2.38017700 & -2.05966300 & -0.72885400\end{array}$




\begin{tabular}{|c|c|c|c|}
\hline $\mathrm{H}$ & -6.69636100 & 0.25745400 & -0.55596100 \\
\hline $\mathrm{H}$ & -6.27001000 & 2.66618400 & -0.09719700 \\
\hline $\mathrm{H}$ & -3.94003000 & 3.45470800 & 0.28347900 \\
\hline $\mathrm{H}$ & -2.05532300 & 1.85203600 & 0.20460200 \\
\hline $\mathrm{C}$ & 1.75386300 & 0.12780400 & 0.51164200 \\
\hline $\mathrm{C}$ & 3.61508900 & 1.36377600 & 0.85689900 \\
\hline $\mathrm{C}$ & 3.95546700 & 0.05911900 & 1.01716000 \\
\hline $\mathrm{H}$ & 4.19855700 & 2.26785100 & 0.93078700 \\
\hline $\mathrm{H}$ & 4.89657500 & -0.40820200 & 1.26038700 \\
\hline $\mathrm{N}$ & 2.80277400 & -0.68646900 & 0.80315200 \\
\hline $\mathrm{N}$ & 2.26146200 & 1.38880600 & 0.54786900 \\
\hline $\mathrm{Au}$ & -0.18504600 & -0.38710600 & 0.08930500 \\
\hline $\mathrm{C}$ & 1.50276000 & 2.59880100 & 0.30512500 \\
\hline $\mathrm{C}$ & 1.42382200 & 3.08970000 & -1.01358500 \\
\hline $\mathrm{C}$ & 0.89476400 & 3.24086400 & 1.40297600 \\
\hline $\mathrm{C}$ & 0.68842100 & 4.26434500 & -1.21603300 \\
\hline $\mathrm{C}$ & 0.17371600 & 4.41251100 & 1.13860400 \\
\hline $\mathrm{C}$ & 0.06895900 & 4.91902400 & -0.15466500 \\
\hline $\mathrm{H}$ & 0.60512600 & 4.67317600 & -2.21842000 \\
\hline $\mathrm{H}$ & -0.30527000 & 4.93869300 & 1.95901400 \\
\hline $\mathrm{H}$ & -0.49089300 & 5.83258700 & -0.33473700 \\
\hline $\mathrm{C}$ & 2.74904500 & -2.13172000 & 0.89433700 \\
\hline $\mathrm{C}$ & 3.01008700 & -2.88954000 & -0.26492200 \\
\hline $\mathrm{C}$ & 2.47416200 & -2.71483700 & 2.14775600 \\
\hline $\mathrm{C}$ & 2.97316200 & -4.28383400 & -0.13912300 \\
\hline $\mathrm{C}$ & 2.45037700 & -4.11422900 & 2.21087000 \\
\hline $\mathrm{C}$ & 2.69466800 & -4.89293100 & 1.08189600 \\
\hline $\mathrm{H}$ & 3.16997800 & -4.90162100 & -1.01019400 \\
\hline $\mathrm{H}$ & 2.24503500 & -4.60025600 & 3.16027600 \\
\hline $\mathrm{H}$ & 2.66190600 & -5.97674900 & 1.15011400 \\
\hline $\mathrm{C}$ & 1.00351500 & 2.72421600 & 2.83482300 \\
\hline $\mathrm{H}$ & 1.58355900 & 1.79592300 & 2.82177100 \\
\hline $\mathrm{C}$ & 1.75994200 & 3.72226600 & 3.73458900 \\
\hline $\mathrm{H}$ & 1.87337400 & 3.31245300 & 4.74450000 \\
\hline $\mathrm{H}$ & 2.75951700 & 3.93901400 & 3.34169000 \\
\hline $\mathrm{H}$ & 1.22288800 & 4.67355200 & 3.82023300 \\
\hline $\mathrm{C}$ & -0.37910100 & 2.38336700 & 3.42418300 \\
\hline $\mathrm{H}$ & -1.01493800 & 3.27243100 & 3.50427500 \\
\hline $\mathrm{H}$ & -0.90452800 & 1.64656100 & 2.80617700 \\
\hline $\mathrm{H}$ & -0.26803900 & 1.96608800 & 4.43141500 \\
\hline $\mathrm{C}$ & 2.21895600 & -1.89464500 & 3.40946600 \\
\hline $\mathrm{C}$ & 3.27986200 & -2.18066400 & 4.49116400 \\
\hline $\mathrm{H}$ & 4.29301400 & -1.99255600 & 4.11901100 \\
\hline $\mathrm{H}$ & 3.11402700 & -1.53927000 & 5.36399100 \\
\hline $\mathrm{H}$ & 3.23843600 & -3.22126500 & 4.83160400 \\
\hline $\mathrm{C}$ & 3.33959800 & -2.25753500 & -1.61420500 \\
\hline $\mathrm{H}$ & 3.28499500 & -1.16961900 & -1.50574600 \\
\hline $\mathrm{C}$ & 2.10108000 & 2.40575500 & -2.19751100 \\
\hline $\mathrm{C}$ & 3.15125400 & 3.32496700 & -2.85269200 \\
\hline $\mathrm{H}$ & 2.68967500 & 4.22020900 & -3.28415200 \\
\hline $\mathrm{H}$ & 3.90405300 & 3.65489000 & -2.12818000 \\
\hline $\mathrm{H}$ & 3.66730400 & 2.79559600 & -3.66154800 \\
\hline $\mathrm{C}$ & 2.31573200 & -2.65509600 & -2.69517600 \\
\hline $\mathrm{H}$ & 1.29765200 & -2.38020300 & -2.39776200 \\
\hline $\mathrm{H}$ & 2.54571900 & -2.14683200 & -3.63853400 \\
\hline $\mathrm{H}$ & 2.33115400 & -3.73374100 & -2.88826300 \\
\hline $\mathrm{C}$ & 4.77596700 & -2.60248300 & -2.05619400 \\
\hline $\mathrm{H}$ & 5.01312200 & -2.10314000 & -3.00234900 \\
\hline $\mathrm{H}$ & 5.51294800 & -2.28468900 & -1.31023600 \\
\hline $\mathrm{H}$ & 4.90065900 & -3.68054600 & -2.20779300 \\
\hline $\mathrm{C}$ & 0.79556900 & -2.12199000 & 3.95579100 \\
\hline $\mathrm{H}$ & 0.03733600 & -1.85643600 & 3.20988800 \\
\hline $\mathrm{H}$ & 0.63765500 & -3.16710600 & 4.24575900 \\
\hline $\mathrm{H}$ & 0.62640000 & -1.50311200 & 4.84431900 \\
\hline $\mathrm{H}$ & 2.29757300 & -0.83358100 & 3.15199600 \\
\hline $\mathrm{C}$ & 1.06825000 & 1.91584100 & -3.23190100 \\
\hline $\mathrm{H}$ & 1.57189000 & 1.38818100 & -4.04990200 \\
\hline
\end{tabular}

$\begin{array}{lrrr}\mathrm{H} & 0.34529000 & 1.22977600 & -2.77600500 \\ \mathrm{H} & 0.51046200 & 2.75144300 & -3.66967100 \\ \mathrm{H} & 2.63052500 & 1.52232300 & -1.82635700 \\ \mathrm{C} & -1.91928800 & -3.42194000 & -0.80027200 \\ \mathrm{C} & -2.07798000 & -4.17842200 & -1.98070900 \\ \mathrm{C} & -1.28986600 & -4.00940200 & 0.31863100 \\ \mathrm{C} & -1.59840000 & -5.48113500 & -2.04960200 \\ \mathrm{H} & -2.56602000 & -3.72891000 & -2.84061300 \\ \mathrm{C} & -0.79383800 & -5.30301000 & 0.23569100 \\ \mathrm{H} & -1.17760300 & -3.43145500 & 1.22941400 \\ \mathrm{C} & -0.94168300 & -6.04410000 & -0.94439300 \\ \mathrm{H} & -1.71144500 & -6.06030700 & -2.95878800 \\ \mathrm{H} & -0.28487200 & -5.75866300 & 1.07693200 \\ \mathrm{O} & -5.82128600 & -2.02607300 & -1.61526700 \\ \mathrm{O} & -5.15770900 & -2.49525800 & 0.48339500 \\ \mathrm{C} & -6.58452000 & -3.18459100 & -1.20537000 \\ \mathrm{H} & -7.60062300 & -2.85460100 & -0.96880300 \\ \mathrm{H} & -6.61239000 & -3.89844200 & -2.03110400 \\ \mathrm{C} & -5.82057600 & -3.68729300 & 0.02346000 \\ \mathrm{H} & -5.07564100 & -4.45016400 & -0.23257000 \\ \mathrm{H} & -6.45659300 & -4.04795500 & 0.83252200 \\ \mathrm{C} & -4.89167800 & -1.74348900 & -0.64121200 \\ \mathrm{H} & -3.79339400 & -2.12335300 & -1.07699600 \\ \mathrm{C} & -0.35266100 & -7.42049200 & -0.97315200 \\ \mathrm{O} & 0.33861200 & -7.87343900 & -0.08195100 \\ \mathrm{O} & -0.66990100 & -8.08891500 & -2.09502300 \\ \mathrm{C} & -0.13114100 & -9.42212600 & -2.19950000 \\ \mathrm{H} & -0.48811000 & -9.80462600 & -3.15515900 \\ \mathrm{H} & 0.96089900 & -9.39277500 & -2.17675900 \\ \mathrm{H} & -0.49129500 & -10.04120100 & -1.37448300 \\ \mathrm{Imaginary} & \mathrm{Frequency}=-565.6453 & \\ & & & \end{array}$

\section{Int1d}

$\begin{array}{lcrr}\mathrm{C} & 2.8336012 & 1.6965363 & -0.7242660 \\ \mathrm{C} & 3.5784352 & 2.7873783 & -0.2128780 \\ \mathrm{C} & 4.9658922 & 2.6883043 & -0.1514250 \\ \mathrm{C} & 5.5939652 & 1.5243443 & -0.5870440 \\ \mathrm{C} & 4.8823222 & 0.4425983 & -1.0945260 \\ \mathrm{C} & 3.4964352 & 0.5340253 & -1.1541400 \\ \mathrm{C} & 1.3849122 & 1.7771523 & -0.7984520 \\ \mathrm{C} & 0.3324572 & 2.4187733 & -0.9953190 \\ \mathrm{H} & 5.5541032 & 3.5160233 & 0.2211300 \\ \mathrm{H} & 5.4079232 & -0.4434687 & -1.4267180 \\ \mathrm{H} & 2.9191332 & -0.2991407 & -1.5412400 \\ \mathrm{C} & -0.6292998 & -1.7937997 & 0.2179950 \\ \mathrm{C} & -0.8564238 & -4.0244157 & 0.4509820 \\ \mathrm{C} & -1.9330408 & -3.4389467 & 1.0371920 \\ \mathrm{H} & -0.5787838 & -5.0608907 & 0.3419770 \\ \mathrm{H} & -2.7878448 & -3.8602527 & 1.5421520 \\ \mathrm{~N} & -1.7782788 & -2.0671767 & 0.8863040 \\ \mathrm{~N} & -0.0638288 & -2.9995497 & -0.0475920 \\ \mathrm{Au} & 0.1383232 & 0.0142953 & -0.2905610 \\ \mathrm{C} & 1.1951702 & -3.1961247 & -0.7399070 \\ \mathrm{C} & 1.1812172 & -3.2894407 & -2.1468170 \\ \mathrm{C} & 2.3721782 & -3.3000847 & 0.0287590 \\ \mathrm{C} & 2.4140442 & -3.4736097 & -2.7859410 \\ \mathrm{C} & 3.5735092 & -3.4900717 & -0.6668170 \\ \mathrm{C} & 3.5972222 & -3.5726187 & -2.0570890 \\ \mathrm{H} & 2.4458022 & -3.5513247 & -3.8685130 \\ \mathrm{H} & 4.5016052 & -3.5801977 & -0.1102980 \\ \mathrm{H} & 4.5403352 & -3.7253257 & -2.5743440 \\ \mathrm{C} & -2.7236698 & -1.0827237 & 1.3749350 \\ \mathrm{C} & -3.8013388 & -0.7193897 & 0.5424390 \\ \mathrm{C} & -2.5372218 & -0.5578797 & 2.6694350 \\ \mathrm{C} & -4.7100578 & 0.2179883 & 1.0484830\end{array}$




\begin{tabular}{|c|c|c|c|}
\hline $\mathrm{C}$ & -3.4781828 & 0.3770183 & 3.1193060 \\
\hline $\mathrm{C}$ & -4.5516668 & 0.7619333 & 2.3205370 \\
\hline & -5.5560638 & 0.5218183 & 0.4392300 \\
\hline & -3.3712268 & 0.8038853 & 4.1119870 \\
\hline & -5.2721068 & 1.4843613 & 2.6936900 \\
\hline & 2.3824092 & -3.2278107 & 1.5531920 \\
\hline & 1.3576012 & -3.0517987 & 1.8962950 \\
\hline $\mathrm{C}$ & 2.8477312 & -4.5614147 & 2.1724920 \\
\hline $\mathrm{H}$ & 2.7988152 & -4.5090947 & 3.2657870 \\
\hline & 2.2216182 & -5.3983877 & 1.8438920 \\
\hline & 3.8826812 & -4.7922127 & 1.8968330 \\
\hline C & 3.2396032 & -2.0523517 & 2.0623950 \\
\hline 1 & 4.2937692 & -2.1734637 & 1.7893780 \\
\hline $\mathrm{H}$ & 2.8945052 & -1.0974957 & 1.6505660 \\
\hline $\mathrm{H}$ & 3.1865802 & -1.9929777 & 3.1552740 \\
\hline $\mathrm{C}$ & -1.3837708 & -0.9702387 & 3.5794760 \\
\hline $\mathrm{C}$ & -1.8973908 & -1.6742047 & 4.8515400 \\
\hline $\mathrm{H}$ & -2.5083388 & -2.5503037 & 4.6072540 \\
\hline $\mathrm{H}$ & -1.0546498 & -2.0086567 & 68330 \\
\hline $\mathrm{H}$ & -2.5094558 & -1.0023427 & 5.4634170 \\
\hline $\mathrm{C}$ & -4.0113448 & -1.3047517 & 1110 \\
\hline $\mathrm{H}$ & -3.1911968 & -1.9992687 & 02490 \\
\hline 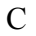 & -0.0988108 & -3.2171197 & -2.97 \\
\hline 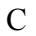 & -0.3417068 & -4.5304477 & -3.7457290 \\
\hline 1 & 0.4501562 & -4.7198407 & 7650 \\
\hline I & -0.3816018 & -5.3910557 & 0460 \\
\hline $\mathrm{H}$ & -1.2 & -4.4 & 9770 \\
\hline $\mathrm{C}$ & -3.9639608 & -0.2 & 66240 \\
\hline H & -3.01 & & 0370 \\
\hline $\mathrm{H}$ & -4.0601988 & & 720 \\
\hline $\mathrm{H}$ & & & \\
\hline $\mathrm{C}$ & & & \\
\hline $\mathrm{H}$ & & & 490 \\
\hline $\mathrm{H}$ & & & 480 \\
\hline $\mathrm{H}$ & & & 880 \\
\hline $\mathrm{C}$ & -0.4 & 0.2 & 3.9 \\
\hline $\mathrm{H}$ & -0.0 & 973 & 3.0 \\
\hline $\mathrm{H}$ & -1.0 & 0.9 & 80 \\
\hline $\mathrm{H}$ & 0.35 & -0.0 & 390 \\
\hline 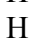 & -0.75 & -1.6 & 5610 \\
\hline C & -0.09 & -2.0 & -3.9296560 \\
\hline $\mathrm{H}$ & -1.0 & -1.9 & 86770 \\
\hline $\mathrm{H}$ & 0.02 & 787 & 82760 \\
\hline $\mathrm{H}$ & 0.7233922 & 8017 & -4.6588790 \\
\hline $\mathrm{H}$ & -0.9437948 & -3.0829867 & -2.2915280 \\
\hline $\mathrm{C}$ & -0.8275798 & 3.2055753 & -1.1636420 \\
\hline $\mathrm{C}$ & -1.5571078 & 3.1919593 & -2.3718400 \\
\hline $\mathrm{C}$ & -1.2375228 & 4.0750153 & -0.1193870 \\
\hline $\mathrm{C}$ & -2.6635228 & 4.0113673 & -2.5422730 \\
\hline $\mathrm{H}$ & -1.2432538 & 2.5351233 & -3.1772350 \\
\hline $\mathrm{C}$ & -2.3343988 & 4.8942213 & -0.2892920 \\
\hline $\mathrm{H}$ & -0.6583318 & 4.1041823 & 0.7981420 \\
\hline $\mathrm{C}$ & -3.0603018 & 3453 & -1.4 \\
\hline $\mathrm{H}$ & -3.2061818 & 3.9 & 4290 \\
\hline $\mathrm{H}$ & -2.6 & & \\
\hline $\mathrm{O}$ & & & \\
\hline $\mathrm{C}$ & & & \\
\hline $\mathrm{H}$ & & & \\
\hline $\mathrm{H}$ & & & -2 \\
\hline $\mathrm{H}$ & -4.2681698 & 6.0997293 & -3.6073150 \\
\hline $\mathrm{O}$ & 3.8258052 & 5.0389783 & 0.6022060 \\
\hline $\mathrm{O}$ & 2.0592782 & 3.8210283 & 1.4115470 \\
\hline $\mathrm{C}$ & 3.4485872 & 5.5956563 & 1.8660820 \\
\hline $\mathrm{H}$ & 4.3573092 & 5.9164003 & 2.3796950 \\
\hline $\mathrm{H}$ & 2.7818142 & 6.4564603 & 1.7231740 \\
\hline$C$ & 2.7270252 & 4.4273683 & 2.5261900 \\
\hline $\mathrm{H}$ & 1.9687792 & 4.7194813 & 3.2560520 \\
\hline
\end{tabular}

$\begin{array}{llll}\mathrm{H} & 3.4321862 & 3.7242343 & 2.9892270 \\ \mathrm{C} & 2.8759472 & 4.0569913 & 0.2591990 \\ \mathrm{H} & 2.2051952 & 4.4303403 & -0.5280100 \\ \mathrm{~N} & 7.0647692 & 1.4336413 & -0.5027440 \\ \mathrm{O} & 7.6664312 & 2.4017513 & -0.0460950 \\ \mathrm{O} & 7.5870972 & 0.3906993 & -0.8918930\end{array}$

\section{TS1d}

$\begin{array}{lrrr}\mathrm{C} & 3.3391600 & 0.4213551 & 0.0467846 \\ \mathrm{C} & 4.4265640 & 1.3251191 & 0.0840906 \\ \mathrm{C} & 5.7418450 & 0.8709651 & 0.1223856 \\ \mathrm{C} & 5.9697530 & -0.4993329 & 0.1843396 \\ \mathrm{C} & 4.9239050 & -1.4208179 & 0.1795556 \\ \mathrm{C} & 3.6158790 & -0.9553279 & 0.1058736\end{array}$

$\begin{array}{llll}\text { C } & 1.9692960 & 0.9421221 & -0.1143884\end{array}$

$\begin{array}{llll}\text { C } & 1.7495860 & 2.2128561 & -0.3682984\end{array}$

$\begin{array}{llll}\mathrm{H} & 6.5787290 & 1.5582401 & 0.1210826\end{array}$

$\begin{array}{llll}\mathrm{H} & 5.1463820 & -2.4799349 & 0.2196056\end{array}$

$\begin{array}{llll}\mathrm{H} & 2.7954610 & -1.6641659 & 0.0774566\end{array}$

$\begin{array}{llll}\text { C } & -1.3034420 & -1.6024479 & 0.0262666\end{array}$

$\begin{array}{llll}\text { C } & -2.4950580 & -3.5238529 & -0.0385934\end{array}$

$\begin{array}{llll}\text { C } & -3.3597280 & -2.5166529 & 0.2468346\end{array}$

$\mathrm{H} \quad-2.6493710 \quad-4.5844289 \quad-0.1594244$

$\begin{array}{llll}\mathrm{H} & -4.4232730 & -2.5186019 & 0.4260846\end{array}$

$\begin{array}{llll}\mathrm{N} & -2.6133170 & -1.3449359 & 0.2839556\end{array}$

$\begin{array}{llll}\mathrm{N} & -1.2388120 & -2.9471259 & -0.1692984\end{array}$

$\mathrm{Au} \quad 0.2785100 \quad-0.2990859 \quad-0.0574404$

$\begin{array}{llll}\text { C } & -0.0263510 & -3.6870599 & -0.4558984\end{array}$

$\begin{array}{llll}\text { C } & 0.3609640 & -3.8516819 & -1.8010184\end{array}$

$\begin{array}{llll}\text { C } & 0.7040890 & -4.2195989 & 0.6263166\end{array}$

$\begin{array}{llll}\text { C } & 1.5414450 & -4.5657949 & -2.0436634\end{array}$

$\begin{array}{llll}\text { C } & 1.8754000 & -4.9252459 & 0.3210916\end{array}$

$\begin{array}{llll}\text { C } & 2.2926640 & -5.0946919 & -0.9972024\end{array}$

$\begin{array}{llll}\mathrm{H} & 1.8736880 & -4.7128609 & -3.0669334\end{array}$

$\begin{array}{llll}\mathrm{H} & 2.4631020 & -5.3557779 & 1.1265366\end{array}$

$\begin{array}{llll}\mathrm{H} & 3.2017600 & -5.6502429 & -1.2101174\end{array}$

$\begin{array}{llll}\text { C } & -3.1766670 & -0.0415439 & 0.5698196\end{array}$

$\begin{array}{llrr}\text { C } & -3.6842410 & 0.7200151 & -0.5018284\end{array}$

$\begin{array}{llll}\text { C } & -3.2273630 & 0.3891851 & 1.9109036\end{array}$

$\begin{array}{llll}\text { C } & -4.2559980 & 1.9601491 & -0.1914814\end{array}$

$\begin{array}{llll}\text { C } & -3.8090430 & 1.6390631 & 2.1593476\end{array}$

$\begin{array}{llll}\text { C } & -4.3190540 & 2.4163681 & 1.1225206\end{array}$

$\begin{array}{llll}\mathrm{H} & -4.6636440 & 2.5725751 & -0.9903194\end{array}$

$\mathrm{H} \quad-3.8733100 \quad 2.0022811 \quad 3.1808666$

$\begin{array}{llll}\mathrm{H} & -4.7760800 & 3.3777851 & 1.3404366\end{array}$

$\begin{array}{llll}\text { C } & 0.2635360 & -4.0729049 & 2.0801826\end{array}$

$\begin{array}{llll}\mathrm{H} & -0.6534780 & -3.4754359 & 2.1025306\end{array}$

$\begin{array}{llll}\text { C } & -0.0711190 & -5.4433789 & 2.7031156\end{array}$

$\begin{array}{llll}\mathrm{H} & -0.4414460 & -5.3148889 & 3.7263836\end{array}$

$\begin{array}{llll}\mathrm{H} & -0.8403470 & -5.9693029 & 2.1267236\end{array}$

$\begin{array}{llll}\mathrm{H} & 0.8114050 & -6.0912319 & 2.7482446\end{array}$

$\begin{array}{llll}\text { C } & 1.3121270 & -3.3215159 & 2.9230536\end{array}$

$\begin{array}{llll}\mathrm{H} & 2.2599600 & -3.8693719 & 2.9728446\end{array}$

$\mathrm{H} \quad 1.5164370 \quad-2.3275209 \quad 2.5092226$

H $\quad 0.9510730 \quad-3.1944589 \quad 3.9499106$

$\begin{array}{llll}\text { C } & -2.6950640 & -0.4429909 & 3.0744426\end{array}$

$\begin{array}{llll}\text { C } & -3.8182910 & -0.8144719 & 4.0629776\end{array}$

$\mathrm{H} \quad-4.6288370 \quad-1.3565579 \quad 3.5635426$

$\begin{array}{llll}\mathrm{H} & -3.4241540 & -1.4526739 & 4.8617496\end{array}$

$\begin{array}{llll}\mathrm{H} & -4.2530010 & 0.0746911 & 4.5329926\end{array}$

$\begin{array}{lllr}\mathrm{H} & -4.2530010 & 0.0746911 & 4.5329926 \\ \mathrm{C} & -3.6474660 & 0.2430011 & -1.9508634\end{array}$

$\mathrm{H} \quad-3.1309800 \quad-0.7214919 \quad-1.9811824$

$\begin{array}{llll}\text { C } & -0.4455880 & -3.2989729 & -2.9725554\end{array}$

$\begin{array}{llll}\mathrm{C} & -0.9477730 & -4.4296909 & -3.8925844 \\ \mathrm{H} & -0.1159470 & -4.9679409 & -4.3602884\end{array}$ 


\begin{tabular}{|c|c|c|c|}
\hline $\mathrm{H}$ & 85280 & -5.1603429 & 664 \\
\hline $\mathrm{H}$ & -1.5685240 & -4.0166059 & -4.6955504 \\
\hline $\mathrm{C}$ & -2.8504660 & 1.2096391 & -2.8484624 \\
\hline $\mathrm{H}$ & -1.8301560 & 1.3498581 & -2.4745294 \\
\hline $\mathrm{H}$ & -2.7896260 & 0.8136521 & -3.8685504 \\
\hline $\mathrm{H}$ & -3.3281900 & 2.1944121 & -2.9044394 \\
\hline $\mathrm{C}$ & -5.0701510 & 0.0198991 & -2.5023034 \\
\hline $\mathrm{H}$ & -5.0250980 & -0.3651249 & -3.5272284 \\
\hline $\mathrm{H}$ & -5.6292470 & -0.6996259 & -1.8938274 \\
\hline $\mathrm{H}$ & -5.6430430 & 0.9538871 & -2.5219014 \\
\hline $\mathrm{C}$ & -1.5342010 & 0.2706731 & 3.7947126 \\
\hline $\mathrm{H}$ & -0.7099130 & 0.4806861 & 3.1031076 \\
\hline $\mathrm{H}$ & -1.8576720 & 1.2188921 & 4.2393146 \\
\hline $\mathrm{H}$ & -1.1460240 & -0.3583009 & 4.60 \\
\hline $\mathrm{H}$ & -2.2950420 & -1.3789989 & \\
\hline 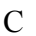 & 0.3573160 & -2.2510799 & -3.7 \\
\hline 1 & -0.25 & -1.8 & -4.5 \\
\hline 1 & 0.6735730 & -1.4 & -3.1 \\
\hline $\mathrm{H}$ & 1.2544600 & -2.6 & -4.2 \\
\hline 1 & -1.3287960 & -2.79 & -2.5 \\
\hline $\mathrm{C}$ & 0.8256850 & 3.3060501 & -0.3 \\
\hline $\mathrm{C}$ & 0.7912450 & 4.2559681 & -1.3 \\
\hline $\mathrm{C}$ & -0.0625340 & 3.4707751 & 0.7 \\
\hline $\mathrm{C}$ & -0.0936310 & 4601 & -1.3 \\
\hline $\mathrm{H}$ & 1.4683110 & 4.1442951 & -2.22 \\
\hline $\mathrm{C}$ & -0.9562390 & 4.5226271 & 0.7823616 \\
\hline $\mathrm{H}$ & -0.0439840 & 2561 & 1.5656436 \\
\hline $\mathrm{C}$ & -0.9807420 & 5.4636731 & -0.2678784 \\
\hline $\mathrm{H}$ & -0.0997730 & 6.0360301 & -2.1704824 \\
\hline $\mathrm{H}$ & -1.6523040 & 4.6491981 & 1.6044536 \\
\hline $\mathrm{O}$ & -1.8833850 & 6.4533271 & -0.1388184 \\
\hline $\mathrm{C}$ & -1.9751750 & 7.4562591 & -1.1521904 \\
\hline $\mathrm{H}$ & -2.2512030 & 7.0177011 & -2.1180634 \\
\hline $\mathrm{H}$ & -2.7609090 & 8.1354881 & -0.8214654 \\
\hline $\mathrm{H}$ & -1.0322590 & 8.0065971 & -1.2496154 \\
\hline $\mathrm{O}$ & 4.9815310 & 3.5338361 & -0.7592574 \\
\hline $\mathrm{O}$ & 3.9842160 & 3.4088431 & 1.2511636 \\
\hline $\mathrm{C}$ & 5.2250370 & 4.8084851 & -0.1148244 \\
\hline $\mathrm{H}$ & 6.2606640 & 4.8156031 & 0.237434 \\
\hline $\mathrm{H}$ & 8950 & 5.60 & -0.8 \\
\hline $\mathrm{C}$ & & 4.81 & \\
\hline $\mathrm{H}$ & 3.261 & 5.29 & 0.7 \\
\hline $\mathrm{H}$ & 4.5761850 & 5.2452001 & 1.958344 \\
\hline $\mathrm{C}$ & 4.1539850 & 2.7898691 & 0.038232 \\
\hline $\mathrm{H}$ & 3.0119660 & 2.7826881 & -0.530553 \\
\hline $\mathrm{N}$ & 7.3590080 & -0.9861249 & 0.258479 \\
\hline $\mathrm{O}$ & 8.2523170 & -0.1421749 & 0.271846 \\
\hline 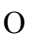 & 7.5279090 & -2.2021849 & 0.300719 \\
\hline
\end{tabular}

$\begin{array}{lrrr}\text { Int1e } & & & \\ \mathrm{C} & 2.6989347 & 1.6037546 & -0.8761483 \\ \mathrm{C} & 3.4621007 & 2.6744706 & -0.3563753 \\ \mathrm{C} & 4.8501657 & 2.5690536 & -0.2961543 \\ \mathrm{C} & 5.4992577 & 1.4090186 & -0.7453703 \\ \mathrm{C} & 4.7420677 & 0.3418186 & -1.2552533 \\ \mathrm{C} & 3.3624677 & 0.4400376 & -1.3123983 \\ \mathrm{C} & 1.2615007 & 1.7101006 & -0.9553823 \\ \mathrm{C} & 0.1604447 & 2.2698856 & -1.1133393 \\ \mathrm{H} & 5.4059587 & 3.4127786 & 0.0898527 \\ \mathrm{H} & 5.2572417 & -0.5475134 & -1.6020043 \\ \mathrm{H} & 2.7816767 & -0.3874564 & -1.7084163 \\ \mathrm{C} & -0.7718903 & -1.8203214 & 0.2551457 \\ \mathrm{C} & -1.0203973 & -4.0382024 & 0.5771857 \\ \mathrm{C} & -2.0514463 & -3.4149664 & 1.2045407\end{array}$

\begin{tabular}{|c|c|c|c|}
\hline $\mathrm{H}$ & -0.7657403 & -5.0824004 & 0. \\
\hline $\mathrm{H}$ & -2.8814443 & -3.8047344 & 1.7723027 \\
\hline & -1.8842013 & -2.0519244 & 0.9980747 \\
\hline $\mathrm{N}$ & -0.2425133 & -3.0443814 & -0.0014623 \\
\hline $\mathrm{Au}$ & -0.0225583 & -0.0355824 & -( \\
\hline $\mathrm{C}$ & 0.9754117 & -3.2876744 & -0.7492533 \\
\hline $\mathrm{C}$ & 0.8892457 & -3.4280964 & -2.1491573 \\
\hline $\mathrm{C}$ & 2.1877617 & -3.3866054 & -0.0370933 \\
\hline $\mathrm{C}$ & 2.0844787 & -3.6608544 & -2.8414993 \\
\hline $\mathrm{C}$ & 3.3484547 & -3.6263304 & -0.7837633 \\
\hline $\mathrm{C}$ & 3.3004267 & -3.7593824 & -2.1692443 \\
\hline H & 2.0605247 & -3.7745164 & -3.9210823 \\
\hline П & 4.3015027 & -3.7 & \\
\hline $\mathrm{H}$ & 4.2131837 & & \\
\hline $\mathrm{C}$ & -2.77 & & \\
\hline $\mathrm{C}$ & -3.9 & & \\
\hline$C$ & -2.4 & -0.4 & 2.7 \\
\hline$C$ & -4.7 & & 1.2 \\
\hline$C$ & -3.38 & & \\
\hline $\mathrm{C}$ & -4.50 & & 797 \\
\hline $\mathrm{H}$ & -5.6 & & 3757 \\
\hline $\mathrm{H}$ & -3.1 & & 927 \\
\hline $\mathrm{H}$ & -5.1 & & 377 \\
\hline $\mathrm{C}$ & 2.27 & & \\
\hline $\mathrm{H}$ & 37 & & \\
\hline $\mathrm{C}$ & 87 & & 067 \\
\hline $\mathrm{H}$ & & & \\
\hline $\mathrm{H}$ & 77 & & \\
\hline Н & 397 & & 847 \\
\hline$C$ & 3.19 & & 307 \\
\hline $\mathrm{H}$ & 67 & & 8027 \\
\hline П & 737 & & 9067 \\
\hline H & 3.20 & -2.0 & 0227 \\
\hline$C$ & -1.2836113 & -0.8 & 8027 \\
\hline $\mathrm{C}$ & -1.7116673 & 324 & 4287 \\
\hline $\mathrm{H}$ & -2.3662003 & 444 & 7597 \\
\hline $\mathrm{H}$ & -0.83 & 54 & 0277 \\
\hline $\mathrm{H}$ & -2.2505873 & 4924 & \\
\hline $\mathrm{C}$ & -4.2266993 & & 0923 \\
\hline $\mathrm{H}$ & & & \\
\hline $\mathrm{C}$ & & & \\
\hline $\mathrm{C}$ & & & \\
\hline $\mathrm{H}$ & & & \\
\hline $\mathrm{H}$ & 3 & & -2 \\
\hline $\mathrm{H}$ & 3 & & -4 \\
\hline $\mathrm{C}$ & 3 & & -1 . \\
\hline $\mathrm{H}$ & 33 & & -1 . \\
\hline $\mathrm{H}$ & 03 & 34 & -2 \\
\hline $\mathrm{H}$ & -5.0 & & -1 \\
\hline $\mathrm{C}$ & -5.5 & 24 & 1953 \\
\hline $\mathrm{H}$ & -5.7 & 34 & 6343 \\
\hline $\mathrm{H}$ & -5.5 & & 357 \\
\hline $\mathrm{H}$ & -6.3 & 484 & 0033 \\
\hline $\mathrm{C}$ & -0.3277623 & 0.3371006 & 9277 \\
\hline $\mathrm{H}$ & 0.0111597 & 7726 & 3067 \\
\hline $\mathrm{H}$ & -0.8098043 & 9816 & 1127 \\
\hline $\mathrm{H}$ & 0.5545897 & 0.0190516 & 4.3830067 \\
\hline $\mathrm{H}$ & -0.7242173 & -1.6243334 & 59197 \\
\hline $\mathrm{C}$ & -0.4412113 & -2.1672244 & -3.9031903 \\
\hline $\mathrm{H}$ & -1.40 & 23504 & 9693 \\
\hline $\mathrm{H}$ & -0.27 & 8604 & -3 \\
\hline $\mathrm{H}$ & 0.33 & & \\
\hline $\mathrm{H}$ & -1.23 & 7914 & 3723 \\
\hline $\mathrm{C}$ & & & \\
\hline $\mathrm{C}$ & -1.78 & 3416 & 8393 \\
\hline $\mathrm{C}$ & & & -0.2216963 \\
\hline $\mathrm{C}$ & -2.9293013 & 3.7584596 & -2.6051573 \\
\hline
\end{tabular}




$\begin{array}{lrrr}\mathrm{H} & -1.4732073 & 2.3250386 & -3.2623543 \\ \mathrm{C} & -2.5777803 & 4.6698306 & -0.3725713 \\ \mathrm{H} & -0.8543173 & 3.9397466 & 0.6838867 \\ \mathrm{C} & -3.3303973 & 4.6133686 & -1.5632133 \\ \mathrm{H} & -3.4911733 & 3.7065356 & -3.5299803 \\ \mathrm{H} & -2.9048773 & 5.3449626 & 0.4112977 \\ \mathrm{O} & -4.4117953 & 5.4178666 & -1.6051713 \\ \mathrm{C} & -5.2106153 & 5.4501446 & -2.7869373 \\ \mathrm{H} & -5.6539003 & 4.4685946 & -2.9934903 \\ \mathrm{H} & -6.0025643 & 6.1720806 & -2.5864683 \\ \mathrm{H} & -4.6243333 & 5.7805756 & -3.6523833 \\ \mathrm{O} & 3.7220567 & 4.9274616 & 0.4663957 \\ \mathrm{O} & 1.9566907 & 3.7106466 & 1.2838547 \\ \mathrm{C} & 3.2822147 & 5.5481826 & 1.6763697 \\ \mathrm{H} & 4.1609817 & 5.9281286 & 2.2021157 \\ \mathrm{H} & 2.5958817 & 6.3783636 & 1.4592307 \\ \mathrm{C} & 2.5691907 & 4.3990096 & 2.3786077 \\ \mathrm{H} & 1.7817667 & 4.7131116 & 3.0681707 \\ \mathrm{H} & 3.2782357 & 3.7458886 & 2.9055237 \\ \mathrm{C} & 2.7702797 & 3.9442756 & 0.1233187 \\ \mathrm{H} & 2.0947297 & 4.3230256 & -0.6575003 \\ \mathrm{O} & 6.8393417 & 1.2257646 & -0.7283263 \\ \mathrm{C} & 7.6731667 & 2.2782576 & -0.2501643 \\ \mathrm{H} & 7.4636497 & 2.5056006 & 0.8021857 \\ \mathrm{H} & 8.6953887 & 1.9106456 & -0.3458273 \\ \mathrm{H} & 7.5526007 & 3.1862796 & -0.8528263\end{array}$

$\begin{array}{lccc}\text { TS1e } & & & \\ \mathrm{C} & -3.28152500 & 0.06899800 & -0.11389600 \\ \mathrm{C} & -4.61249200 & -0.37987300 & -0.29373400 \\ \mathrm{C} & -5.69107500 & 0.48675400 & -0.19396000 \\ \mathrm{C} & -5.48071900 & 1.83262100 & 0.14630800 \\ \mathrm{C} & -4.17116300 & 2.29236000 & 0.35386300 \\ \mathrm{C} & -3.09509700 & 1.41713200 & 0.21883000 \\ \mathrm{C} & -2.16945700 & -0.86176300 & -0.35327500 \\ \mathrm{C} & -2.36654300 & -2.04507500 & -0.89445100 \\ \mathrm{H} & -6.70535100 & 0.13902700 & -0.35733600 \\ \mathrm{H} & -3.98049300 & 3.32890600 & 0.60586400 \\ \mathrm{H} & -2.08590700 & 1.79337900 & 0.35481700 \\ \mathrm{C} & 1.75307800 & 0.16035400 & 0.53764600 \\ \mathrm{C} & 3.59983500 & 1.41781800 & 0.90148300 \\ \mathrm{C} & 3.94256100 & 0.12082200 & 1.10802100 \\ \mathrm{H} & 4.17604900 & 2.32722900 & 0.96635000 \\ \mathrm{H} & 4.87878000 & -0.33350400 & 1.39140200 \\ \mathrm{~N} & 2.79978700 & -0.63715900 & 0.88227100 \\ \mathrm{~N} & 2.25494600 & 1.42524400 & 0.55438700 \\ \mathrm{Au} & -0.17526600 & -0.37916600 & 0.07423000 \\ \mathrm{C} & 1.49707900 & 2.62652900 & 0.27152000 \\ \mathrm{C} & 1.42766000 & 3.08211900 & -1.05997000 \\ \mathrm{C} & 0.88072900 & 3.29802000 & 1.34702200 \\ \mathrm{C} & 0.69407300 & 4.25103400 & -1.29936100 \\ \mathrm{C} & 0.16232200 & 4.46242900 & 1.04643500 \\ \mathrm{C} & 0.06723900 & 4.93412700 & -0.26064400 \\ \mathrm{H} & 0.61719300 & 4.63240500 & -2.31309100 \\ \mathrm{H} & -0.32212000 & 5.01059200 & 1.84918700 \\ \mathrm{H} & -0.49112000 & 5.84250300 & -0.46957000 \\ \mathrm{C} & 2.75582900 & -2.07791400 & 1.02265500 \\ \mathrm{C} & 3.07205900 & -2.87324500 & -0.09661200 \\ \mathrm{C} & 2.44127700 & -2.61924100 & 2.28555000 \\ \mathrm{C} & 3.06296600 & -4.26225100 & 0.08172900 \\ \mathrm{C} & 2.44702800 & -4.01509700 & 2.40311400 \\ \mathrm{C} & 2.75667500 & -4.82881600 & 1.31623100 \\ \mathrm{H} & 3.30519200 & -4.90783500 & -0.75720200 \\ \mathrm{H} & 2.21540600 & -4.46892300 & 3.36234200 \\ \mathrm{H} & 2.76673300 & -5.90907800 & 1.43357500\end{array}$

$\begin{array}{llll}0.97888900 & 2.81948300 & 2.79292400\end{array}$ $\begin{array}{lll}1.55995700 & 1.89205500 & 2.80869700\end{array}$ $\begin{array}{lll}1.72731400 & 3.84159200 & 3.67200500\end{array}$ $\begin{array}{lll}1.83640700 & 3.45723700 & 4.69239900\end{array}$ $\begin{array}{lll}2.72842200 & 4.05170800 & 3.27939800\end{array}$ $\begin{array}{lll}1.18718700 & 4.79323500 & 3.73131400\end{array}$ $\begin{array}{lll}-0.40732600 & 2.49172200 & 3.38098800\end{array}$ $\begin{array}{lll}-1.04545100 & 3.38153600 & 3.43153300\end{array}$ $\begin{array}{lll}-0.92605200 & 1.73622900 & 2.78046100\end{array}$ $\begin{array}{lll}-0.30301300 & 2.10344100 & 4.40055300\end{array}$ $\begin{array}{lll}2.11546500 & -1.75855500 & 3.50282400\end{array}$ $\begin{array}{lll}3.14494400 & -1.96693400 & 4.63153100\end{array}$ $\begin{array}{lll}4.16427600 & -1.75644000 & 4.28917700\end{array}$ $\begin{array}{llll}2.92530000 & -1.30094500 & 5.47358000\end{array}$ $\begin{array}{llll}3.12644500 & -2.99554900 & 5.00859700\end{array}$ $\begin{array}{llll}3.42589300 & -2.28565500 & -1.45969300\end{array}$ $\begin{array}{llll}3.36597800 & -1.19514600 & -1.38824100\end{array}$ $\begin{array}{lll}2.11396000 & 2.36651500 & -2.21928900\end{array}$ $\begin{array}{llll}3.17687700 & 3.26384200 & -2.88411800\end{array}$ $\begin{array}{llll}2.72550300 & 4.15018200 & -3.34398200\end{array}$ $\begin{array}{lll}3.92245500 & 3.60821400 & -2.15878400\end{array}$ $\begin{array}{llll}3.70018500 & 2.71165400 & -3.67288300\end{array}$ $\begin{array}{lll}2.41950300 & -2.72348500 & -2.54172500\end{array}$ $\begin{array}{lll}1.39781600 & -2.43288400 & -2.27386200\end{array}$ $\begin{array}{lll}2.66883500 & -2.25582900 & -3.50115300\end{array}$ $\begin{array}{llll}2.43379700 & -3.80963300 & -2.68808700\end{array}$ $\begin{array}{lll}4.87015500 & -2.63898900 & -1.86707100\end{array}$ $\begin{array}{lll}5.12097300 & -2.16685400 & -2.82360200\end{array}$ $\begin{array}{llll}5.59384600 & -2.29629600 & -1.11911400\end{array}$ $\begin{array}{lll}5.00153900 & -3.72047300 & -1.98546700\end{array}$ $\begin{array}{llll}0.68053600 & -2.01099600 & 4.00615700\end{array}$ $-0.05599900 \quad-1.80794900 \quad 3.22042300$ $\begin{array}{lll}0.54653000 & -3.04661100 & 4.33918500\end{array}$ $\begin{array}{lll}0.45714300 & -1.35835600 & 4.85781800\end{array}$ $\begin{array}{lll}2.16965000 & -0.70692100 & 3.20456000\end{array}$ $\begin{array}{lll}1.08988800 & 1.85710700 & -3.25282500\end{array}$ $\begin{array}{llll}1.59958300 & 1.30523400 & -4.05094400\end{array}$ $\begin{array}{llll}0.35716900 & 1.18812700 & -2.78750000\end{array}$ $\begin{array}{lll}0.54334800 & 2.68503800 & -3.71867300\end{array}$ $\begin{array}{llll}2.63398300 & 1.48962200 & -1.82050600\end{array}$ $\begin{array}{lll}-1.88390900 & -3.38651000 & -1.06112600\end{array}$ $-2.02589400-4.05538800 \quad-2.29438700$ $\begin{array}{lll}-1.27469900 & -4.08156400 & 0.01320400\end{array}$ $\begin{array}{lll}-1.57671000 & -5.35881200 & -2.46716800\end{array}$ $-2.49314600 \quad-3.53646400 \quad-3.12682000$ $\begin{array}{lll}-0.80985300 & -5.37186900 & -0.15447000\end{array}$ $\begin{array}{lll}-1.16182400 & -3.58104900 & 0.96918200\end{array}$ $\begin{array}{lll}-0.95794800 & -6.02605000 & -1.39402900\end{array}$ $\begin{array}{lll}-1.69734200 & -5.84296900 & -3.42891200\end{array}$ $\begin{array}{lll}-0.32581900 & -5.90532600 & 0.65635500\end{array}$ $\begin{array}{lll}-0.47643000 & -7.28459700 & -1.44949300\end{array}$ $\begin{array}{lll}-0.59401600 & -8.02281800 & -2.66529400\end{array}$ $\begin{array}{lll}-0.05305100 & -7.53074200 & -3.48221600\end{array}$ $\begin{array}{lll}-0.14372000 & -8.99496600 & -2.46346900\end{array}$ $\begin{array}{lll}-1.64497300 & -8.15799800 & -2.94674600\end{array}$ $\begin{array}{lll}-5.80670600 & -2.06243700 & -1.58595000\end{array}$ $-4.97618500 \quad-2.67755500 \quad 0.40998200$ $-6.48334100 \quad-3.29193500-1.23537600$ $\begin{array}{llll}-7.49353600 & -3.03881500 & -0.89961900\end{array}$ $-6.53743000 \quad-3.92773400-2.12121100$ $\begin{array}{lll}-5.60817900 & -3.86051800 & -0.11494000\end{array}$ $\begin{array}{lll}-4.84457500 & -4.55095900 & -0.49079100\end{array}$ $\begin{array}{lll}-6.16536900 & -4.32779900 & 0.69768300\end{array}$ $\begin{array}{lll}-4.83985700 & -1.80875800 & -0.64653000\end{array}$ $\begin{array}{lll}-3.72189500 & -2.08183600 & -1.20211000\end{array}$ $\begin{array}{lll}-6.59725700 & 2.59004200 & 0.25124700\end{array}$ $\begin{array}{lll}-6.46521600 & 3.96262800 & 0.60820000\end{array}$ 
$\begin{array}{lrrr}\mathrm{H} & -5.99902700 & 4.07448000 & 1.59490700 \\ \mathrm{H} & -5.88187100 & 4.51439600 & -0.13921400 \\ \mathrm{H} & -7.48081700 & 4.35843600 & 0.63957300\end{array}$ Imaginary frequency $=-642.4422$

$\begin{array}{lrrr}\text { Int1f } & & & \\ \mathrm{C} & -3.99809900 & 0.43567300 & 0.36017100 \\ \mathrm{C} & -4.49807500 & 0.33664500 & -0.96521600 \\ \mathrm{C} & -5.34444100 & 1.33665500 & -1.43429100 \\ \mathrm{C} & -5.69194900 & 2.41898100 & -0.61989700 \\ \mathrm{C} & -5.19511600 & 2.52320700 & 0.68298100 \\ \mathrm{C} & -4.35136700 & 1.53598300 & 1.17454400 \\ \mathrm{C} & -3.18350400 & -0.57823800 & 0.91554400 \\ \mathrm{H} & -5.74474300 & 1.25074500 & -2.43749000 \\ \mathrm{H} & -6.36023400 & 3.18375300 & -1.00526600 \\ \mathrm{H} & -5.47181500 & 3.36412000 & 1.31090300 \\ \mathrm{H} & -3.96040300 & 1.59095100 & 2.18539200 \\ \mathrm{C} & 1.37429700 & -0.02373600 & 0.44665500 \\ \mathrm{C} & 3.25561400 & 1.18839500 & 0.18162700 \\ \mathrm{C} & 3.58983100 & -0.12143600 & 0.04541000 \\ \mathrm{H} & 3.85106900 & 2.08601200 & 0.12752900 \\ \mathrm{H} & 4.53661900 & -0.59957700 & -0.15005200 \\ \mathrm{~N} & 2.42231000 & -0.85378600 & 0.21143300 \\ \mathrm{~N} & 1.89017000 & 1.23197200 & 0.42973600 \\ \mathrm{Au} & -0.54563300 & -0.57805300 & 0.80794900 \\ \mathrm{C} & 1.13904600 & 2.45195300 & 0.65171500 \\ \mathrm{C} & 0.59868000 & 3.11997900 & -0.46535600 \\ \mathrm{C} & 1.00914600 & 2.92693300 & 1.97245700 \\ \mathrm{C} & -0.10449900 & 4.30589100 & -0.21935200 \\ \mathrm{C} & 0.29442800 & 4.11746900 & 2.15473900 \\ \mathrm{C} & -0.25757000 & 4.79946100 & 1.07359500 \\ \mathrm{H} & -0.53080700 & 4.85405800 & -1.05404900 \\ \mathrm{H} & 0.17617500 & 4.51860000 & 3.15680100 \\ \mathrm{H} & -0.80244800 & 5.72478100 & 1.23854400 \\ \mathrm{C} & 2.34527000 & -2.30086700 & 0.15232000 \\ \mathrm{C} & 2.11693000 & -2.90961900 & -1.09800700 \\ \mathrm{C} & 2.52220900 & -3.03099500 & 1.34506800 \\ \mathrm{C} & 2.05354600 & -4.30832200 & -1.12381500\end{array}$

\begin{tabular}{rrr}
2.44619900 & -4.42664000 & 1.25550200 \\
2.21237200 & -5.05957300 & 0.03770000 \\
1.88318700 & -4.81608100 & -2.06824700 \\
2.57897100 & -5.02501300 & 2.15181200 \\
2.16152400 & -6.14372300 & -0.00785500 \\
1.62239400 & 2.21829700 & 3.17700400 \\
2.10417300 & 1.29978400 & 2.82638500 \\
2.71476700 & 3.08359800 & 3.83747900 \\
3.17863200 & 2.54108300 & 4.66879800 \\
3.50231700 & 3.34979100 & 3.12382600 \\
2.30040800 & 4.01541600 & 4.23814300 \\
0.54947200 & 1.80319200 & 4.20252600 \\
0.03720200 & 2.67393000 & 4.62669900 \\
-0.20788900 & 1.15582100 & 3.74587300 \\
1.01064600 & 1.25527700 & 5.03195600 \\
2.80599400 & -2.37337500 & 2.69271900 \\
4.19727500 & -2.77109500 & 3.22579200 \\
4.98849500 & -2.51483400 & 2.51255100 \\
4.40674400 & -2.25102600 & 4.16721800 \\
4.26017900 & -3.84762900 & 3.41933300 \\
1.97110900 & -2.11982200 & -2.39557700 \\
1.98976900 & -1.05199200 & -2.15418900 \\
0.78177400 & 2.62966100 & -1.89864000 \\
1.68875600 & 3.59001100 & -2.69580900 \\
1.23513000 & 4.58336000 & -2.78664900 \\
2.66547900 & 3.71461900 & -2.21496300 \\
1.85559600 & 3.20468700 & -3.70796300 \\
0.62613800 & -2.39992300 & -3.09372100 \\
-0.22273800 & -2.14060700 & -2.45131400 \\
0.55619700 & -1.80455300 & -4.01197100 \\
0.52959000 & -3.45369600 & -3.37808100 \\
3.15531000 & -2.39613400 & -3.34473500 \\
3.06693900 & -1.78758900 & -4.25174200 \\
4.11425800 & -2.16068200 & -2.86986800 \\
-2.4485800 & -3.44810300 & -3.64978500 \\
-2.39910500 & -2.35648100 & 1.98689000 \\
-4.08863300 & -0.82629500 & -1.85426400 \\
1.70573600 & -2.69084200 & 3.72401400 \\
0.72165200 & -2.36801700 & 3.36498300 \\
1.65068900 & -3.76371100 & 3.93925500 \\
1.91053000 & -2.17368300 & 4.66815800 \\
2.81122100 & -1.28743000 & 2.55268300 \\
-0.56516300 & 2.41807400 & -2.61642200 \\
-0.38822200 & 2.04998100 & -3.63388800 \\
-1.19062600 & 1.68609800 & -2.09429100 \\
-1.13077900 & 3.35299500 & -2.70114600 \\
1.28310800 & 1.65666300 & -1.86909000 \\
-2.75402900 & -0.63586100 & -2.35200700 \\
-4.94618800 & -0.91460600 & -2.96223000 \\
-2.83013000 & -0.57647100 & -3.78366200 \\
-2.85970400 & 0.46662400 & -4.12413100 \\
-1.94588400 & -1.06933600 & -4.19402900 \\
\hline .6433100 & -2.39335600 & -4.09484300 \\
\hline
\end{tabular}




\begin{tabular}{|c|c|c|c|}
\hline $\mathrm{C}$ & -2.01342700 & -0.83910900 & -0.63264000 \\
\hline $\mathrm{C}$ & -2.02459900 & -2.09446200 & -0.87733500 \\
\hline $\mathrm{H}$ & -6.49735500 & -0.05579400 & -1.33201600 \\
\hline $\mathrm{H}$ & -6.24192200 & 2.40707400 & -1.10368600 \\
\hline $\mathrm{H}$ & -4.00100000 & 3.38146600 & -0.63551700 \\
\hline $\mathrm{H}$ & -2.02698700 & 1.89655300 & -0.39760400 \\
\hline $\mathrm{C}$ & 1.88229600 & 0.22584400 & 0.38559000 \\
\hline $\mathrm{C}$ & 3.63971900 & 1.52378600 & 0.95035600 \\
\hline $\mathrm{C}$ & 4.07121900 & 0.23581600 & 0.93089700 \\
\hline $\mathrm{H}$ & 4.15458800 & 2.44596900 & 1.16888300 \\
\hline $\mathrm{H}$ & 5.04004300 & -0.19501700 & 1.12848600 \\
\hline $\mathrm{N}$ & 2.97959500 & -0.54927700 & 0.58215100 \\
\hline $\mathrm{N}$ & 2.29276000 & 1.50005800 & 0.61329000 \\
\hline $\mathrm{Au}$ & -0.00406900 & -0.36450300 & -0.13206200 \\
\hline $\mathrm{C}$ & 1.44760000 & 2.67325000 & 0.51342000 \\
\hline $\mathrm{C}$ & 1.35140000 & 3.32640200 & -0.73239300 \\
\hline $\mathrm{C}$ & 0.76728400 & 3.11082600 & 1.66792700 \\
\hline $\mathrm{C}$ & 0.52524200 & 4.45564000 & -0.79877500 \\
\hline $\mathrm{C}$ & -0.04501400 & 4.24473000 & 1.53886500 \\
\hline $\mathrm{C}$ & -0.16631400 & 4.91031300 & 0.32148500 \\
\hline $\mathrm{H}$ & 0.42688500 & 4.98829000 & -1.73997400 \\
\hline $\mathrm{H}$ & -0.58492200 & 4.61328700 & 2.40586000 \\
\hline $\mathrm{H}$ & -0.79628600 & 5.79243000 & 0.24726600 \\
\hline $\mathrm{C}$ & 3.02180200 & -1.99117800 & 0.43959400 \\
\hline $\mathrm{C}$ & 3.35430000 & -2.52997800 & -0.81966800 \\
\hline $\mathrm{C}$ & 2.74962300 & -2.78645100 & 1.57065800 \\
\hline $\mathrm{C}$ & 3.39865300 & -3.92591300 & -0.92507800 \\
\hline $\mathrm{C}$ & 2.81020300 & -4.17580700 & 1.40354000 \\
\hline $\mathrm{C}$ & 3.12949700 & -4.74086100 & 0.17155600 \\
\hline $\mathrm{H}$ & 3.65330300 & -4.37960600 & -1.87834000 \\
\hline $\mathrm{H}$ & 2.60922500 & -4.82278400 & 2.25228900 \\
\hline $\mathrm{H}$ & 3.17558500 & -5.82128500 & 0.06726300 \\
\hline $\mathrm{C}$ & 0.88054400 & 2.40925900 & 3.01851600 \\
\hline $\mathrm{H}$ & 1.58630600 & 1.57856400 & 2.91690000 \\
\hline $\mathrm{C}$ & 1.44229900 & 3.35282200 & 4.10084100 \\
\hline $\mathrm{H}$ & 1.57133900 & 2.81135000 & 5.04456000 \\
\hline $\mathrm{H}$ & 2.41480000 & 3.76439900 & 3.80917400 \\
\hline $\mathrm{H}$ & 0.76786000 & 4.19470200 & 4.29192500 \\
\hline $\mathrm{C}$ & -0.47082500 & 1.80775400 & 3.45356200 \\
\hline $\mathrm{H}$ & -1.23033500 & 2.58571000 & 3.59150000 \\
\hline $\mathrm{H}$ & -0.84841900 & 1.09637200 & 2.70974700 \\
\hline $\mathrm{H}$ & -0.36099200 & 1.27805800 & 4.40660600 \\
\hline $\mathrm{C}$ & 2.40804900 & -2.20198800 & 2.93851800 \\
\hline $\mathrm{C}$ & 3.47794900 & -2.56597900 & 3.98751500 \\
\hline $\mathrm{H}$ & 4.47362400 & -2.22832200 & 3.67928300 \\
\hline $\mathrm{H}$ & 3.24218900 & -2.09615700 & 4.94895500 \\
\hline $\mathrm{H}$ & 3.52975000 & -3.64823100 & 4.15025700 \\
\hline $\mathrm{C}$ & 3.66519200 & -1.66786600 & -2.03987300 \\
\hline $\mathrm{H}$ & 3.59012800 & -0.61570000 & -1.74680500 \\
\hline $\mathrm{C}$ & 2.10090400 & 2.86082600 & -1.97752000 \\
\hline $\mathrm{C}$ & 3.09812100 & 3.93010500 & -2.46710600 \\
\hline $\mathrm{H}$ & 2.58438400 & 4.84481500 & -2.78303600 \\
\hline $\mathrm{H}$ & 3.81212600 & 4.20318500 & -1.68229900 \\
\hline $\mathrm{H}$ & 3.66441100 & 3.55418700 & -3.32643900 \\
\hline $\mathrm{C}$ & 2.64031300 & -1.89791500 & -3.16804600 \\
\hline $\mathrm{H}$ & 1.62037100 & -1.68539300 & -2.82728200 \\
\hline $\mathrm{H}$ & 2.85845300 & -1.24222900 & -4.01862000 \\
\hline $\mathrm{H}$ & 2.66718000 & -2.93155600 & -3.53092500 \\
\hline $\mathrm{C}$ & 5.10485400 & -1.89851100 & -2.54137900 \\
\hline $\mathrm{H}$ & 5.32502500 & -1.23106800 & -3.38194300 \\
\hline $\mathrm{H}$ & 5.84020700 & -1.70554300 & -1.75247800 \\
\hline $\mathrm{H}$ & 5.25109900 & -2.92754100 & -2.88790400 \\
\hline $\mathrm{C}$ & 1.00392500 & -2.63413100 & 3.40448600 \\
\hline $\mathrm{H}$ & 0.23597400 & -2.33413800 & 2.68214200 \\
\hline $\mathrm{H}$ & 0.93908500 & -3.72014400 & 3.53551500 \\
\hline $\mathrm{H}$ & 0.76537500 & -2.16957500 & 4.36784500 \\
\hline $\mathrm{H}$ & 239594200 & -1.110680 & 2.85320 \\
\hline
\end{tabular}

$\begin{array}{crrr}\mathrm{C} & 1.13025100 & 2.45164300 & -3.10306000 \\ \mathrm{H} & 1.69035000 & 2.08316200 & -3.96989600 \\ \mathrm{H} & 0.45029800 & 1.65727000 & -2.77433900 \\ \mathrm{H} & 0.52128600 & 3.29877900 & -3.43819300 \\ \mathrm{H} & 2.68277100 & 1.97132400 & -1.71513200 \\ \mathrm{O} & -5.39790600 & -2.35485100 & -2.13795800 \\ \mathrm{O} & -5.07681900 & -2.53858300 & 0.08392000 \\ \mathrm{C} & -6.18892000 & -3.48914800 & -1.71802800 \\ \mathrm{H} & -7.24045100 & -3.18710700 & -1.71785700 \\ \mathrm{H} & -6.04330200 & -4.30748800 & -2.42693300 \\ \mathrm{C} & -5.65416700 & -3.79290200 & -0.31197400 \\ \mathrm{H} & -4.88092600 & -4.57248500 & -0.32233100 \\ \mathrm{H} & -6.42333500 & -4.05186400 & 0.41654000 \\ \mathrm{C} & -4.61049000 & -1.94994900 & -1.07780900 \\ \mathrm{H} & -3.49846500 & -2.37519900 & -1.27749700 \\ \mathrm{H} & -1.61380400 & -3.08798800 & -0.83988800 \\ \mathrm{Imaginary} & \text { Frequency }=-494.1779 & \end{array}$

$\begin{array}{cccc}\text { Int2f } & & & \\ \mathrm{C} & -3.51670800 & -0.21407300 & 0.04983700 \\ \mathrm{C} & -4.03542200 & -0.20598400 & -1.28965400 \\ \mathrm{C} & -4.98112100 & 0.77883700 & -1.70803800 \\ \mathrm{C} & -5.42863900 & 1.73304400 & -0.82563800 \\ \mathrm{C} & -4.94239700 & 1.72471100 & 0.49847000 \\ \mathrm{C} & -4.01059700 & 0.78736400 & 0.91443200 \\ \mathrm{C} & -2.50509600 & -1.14747200 & 0.55899000 \\ \mathrm{H} & -5.34617000 & 0.76081500 & -2.72874800 \\ \mathrm{H} & -6.15009600 & 2.47890300 & -1.14197600 \\ \mathrm{H} & -5.30626800 & 2.46450000 & 1.20639300 \\ \mathrm{H} & -3.63794800 & 0.80257500 & 1.93309400 \\ \mathrm{C} & 1.44278100 & 0.07371700 & 0.18920500 \\ \mathrm{C} & 3.31191300 & 1.35135100 & 0.08159200 \\ \mathrm{C} & 3.70735100 & 0.05316300 & 0.08872200 \\ \mathrm{H} & 3.87712600 & 2.26915000 & 0.04205300 \\ \mathrm{H} & 4.68817100 & -0.39441800 & 0.05652400 \\ \mathrm{~N} & 2.55139400 & -0.71627600 & 0.15321800 \\ \mathrm{~N} & 1.92335600 & 1.34711600 & 0.14450000 \\ \mathrm{Au} & -0.53331200 & -0.52659300 & 0.32926000 \\ \mathrm{C} & 1.11725600 & 2.54954600 & 0.17424100 \\ \mathrm{C} & 0.64582500 & 3.07651300 & -1.04460200 \\ \mathrm{C} & 0.86557900 & 3.15854400 & 1.41976400 \\ \mathrm{C} & -0.10852200 & 4.25478100 & -0.98670000 \\ \mathrm{C} & 0.10507600 & 4.33486200 & 1.41588700 \\ \mathrm{C} & -0.37718900 & 4.87884100 & 0.22865400 \\ \mathrm{H} & -0.48478000 & 4.69263600 & -1.90674300 \\ \mathrm{H} & -0.10992400 & 4.83250500 & 2.35691300 \\ \mathrm{H} & -0.96039500 & 5.79528600 & 0.25014400 \\ \mathrm{C} & 2.54689900 & -2.16379400 & 0.17463300 \\ \mathrm{C} & 2.53788800 & -2.85314700 & -1.05445700 \\ \mathrm{C} & 2.57556100 & -2.82126800 & 1.42010500 \\ \mathrm{C} & 2.54134700 & -4.25230000 & -1.00745100 \\ \mathrm{C} & 2.57842900 & -4.22216100 & 1.40548000 \\ \mathrm{C} & 2.55931500 & -4.93178200 & 0.20821700 \\ \mathrm{H} & 2.53507500 & -4.81762400 & -1.93501200 \\ \mathrm{H} & 2.59992200 & -4.76316200 & 2.34689400 \\ \mathrm{H} & 2.56537100 & -6.01810000 & 0.22213700 \\ \mathrm{C} & 1.37793300 & 2.59304100 & 2.74093000 \\ \mathrm{H} & 1.95381500 & 1.68719800 & 2.52724300 \\ \mathrm{C} & 2.32696900 & 3.58021000 & 3.44916000 \\ \mathrm{H} & 2.72489000 & 3.13145400 & 4.36623800 \\ \mathrm{H} & 3.17390700 & 3.85307600 & 2.80960800 \\ \mathrm{H} & 1.81103500 & 4.50513400 & 3.73026100 \\ & 0.21413600 & 2.17967800 & 3.66308200 \\ -0.40445900 & 3.04072200 & 3.94126200 \\ \mathrm{H} & -0.43088000 & 1.44061000 & 3.17498900\end{array}$




$\begin{array}{lrrr}\mathrm{H} & 0.60219500 & 1.73753400 & 4.58781300 \\ \mathrm{C} & 2.61112500 & -2.07846900 & 2.75254800 \\ \mathrm{C} & 3.90505400 & -2.38656200 & 3.53224400 \\ \mathrm{H} & 4.79645300 & -2.13882200 & 2.94512600 \\ \mathrm{H} & 3.93642600 & -1.80412000 & 4.45986000 \\ \mathrm{H} & 3.96909200 & -3.44623600 & 3.80362100 \\ \mathrm{C} & 2.51386900 & -2.13856700 & -2.40291600 \\ \mathrm{H} & 2.60363500 & -1.06263800 & -2.22427500 \\ \mathrm{C} & 0.93180500 & 2.42690700 & -2.39524700 \\ \mathrm{C} & 1.78271000 & 3.34518000 & -3.29476100 \\ \mathrm{H} & 1.25346000 & 4.27338600 & -3.53782700 \\ \mathrm{H} & 2.72564700 & 3.61815000 & -2.80852000 \\ \mathrm{H} & 2.02149500 & 2.84113000 & -4.23837400 \\ \mathrm{C} & 1.16808500 & -2.36215900 & -3.12229900 \\ \mathrm{H} & 0.33761000 & -2.00994000 & -2.49858400 \\ \mathrm{H} & 1.14989100 & -1.81559900 & -4.07318100 \\ \mathrm{H} & 1.01227800 & -3.42600800 & -3.34137300 \\ \mathrm{C} & 3.69905200 & -2.55119800 & -3.29651900 \\ \mathrm{H} & 3.69041200 & -1.97620800 & -4.22953800 \\ \mathrm{H} & 4.65634600 & -2.37025700 & -2.79601400 \\ \mathrm{H} & 3.65880400 & -3.61341500 & -3.56199000 \\ \mathrm{C} & 1.36225100 & -2.38477700 & 3.60218000 \\ \mathrm{H} & 0.44434500 & -2.11817200 & 3.06643100 \\ \mathrm{H} & 1.30519700 & -3.44721100 & 3.86509600 \\ \mathrm{H} & 1.39073900 & -1.81302600 & 4.53684000 \\ \mathrm{H} & 2.60446500 & -1.00370600 & 2.54600100 \\ \mathrm{C} & -0.37066300 & 2.00667700 & -3.10420400 \\ \mathrm{H} & -0.14015800 & 1.49853200 & -4.04847100 \\ \mathrm{H} & -0.95541600 & 1.32582800 & -2.47469400 \\ \mathrm{H} & -0.99878300 & 2.87350900 & -3.33908000 \\ \mathrm{H} & 1.51016400 & 1.51443900 & -2.22091100 \\ \mathrm{O} & -2.77376400 & -2.11785800 & -2.05837300 \\ \mathrm{O} & -4.19692800 & -1.19244600 & -3.45699700 \\ \mathrm{C} & -2.73574400 & -2.99827900 & -3.22238500 \\ \mathrm{H} & -1.68818700 & -3.14951800 & -3.47898100 \\ \mathrm{H} & -3.19622300 & -3.94298800 & -2.92615300 \\ \mathrm{C} & -3.54002600 & -2.21253800 & -4.26513700 \\ \mathrm{H} & -4.31898200 & -2.79084600 & -4.76159400 \\ \mathrm{H} & -2.92149600 & -1.69762600 & -5.00335000 \\ \mathrm{C} & -3.66055100 & -1.17709100 & -2.25099700 \\ \mathrm{C} & -2.85475600 & -2.25511800 & 1.23125800 \\ \mathrm{H} & -2.11355500 & -2.92731100 & 1.65448700 \\ \mathrm{H} & -3.89781300 & -2.53967400 & 1.38665600 \\ & & & \\ & & & \end{array}$

$\begin{array}{lccc}\text { TS2f } & & & \\ \mathrm{C} & -3.38728600 & 0.05623100 & -0.28601600 \\ \mathrm{C} & -4.44919300 & -0.49476100 & -1.03772700 \\ \mathrm{C} & -5.65114900 & 0.19288400 & -1.22851500 \\ \mathrm{C} & -5.75289300 & 1.48718700 & -0.72399400 \\ \mathrm{C} & -4.67856300 & 2.07586700 & -0.03389400 \\ \mathrm{C} & -3.49313100 & 1.37988200 & 0.17482400 \\ \mathrm{C} & -2.30908400 & -0.89037800 & -0.04813200 \\ \mathrm{H} & -6.46741600 & -0.25345600 & -1.78738600 \\ \mathrm{H} & -6.66194400 & 2.05725600 & -0.88988200 \\ \mathrm{H} & -4.78191700 & 3.08775300 & 0.34663300 \\ \mathrm{H} & -2.67073800 & 1.82694200 & 0.72472000 \\ \mathrm{C} & 1.64791900 & 0.20069800 & 0.14025100 \\ \mathrm{C} & 3.51893400 & 1.46352600 & 0.30403500 \\ \mathrm{C} & 3.90521400 & 0.16131100 & 0.29255900 \\ \mathrm{H} & 4.08985200 & 2.37629200 & 0.36954800 \\ \mathrm{H} & 4.88192400 & -0.29334200 & 0.34417300 \\ \mathrm{~N} & 2.74734300 & -0.59873200 & 0.19255400 \\ \mathrm{~N} & 2.13390400 & 1.46984500 & 0.20954800 \\ \mathrm{Au} & -0.33905500 & -0.36077600 & 0.01689100 \\ \mathrm{C} & 1.32403400 & 2.67096400 & 0.20372600\end{array}$

$\begin{array}{lll}1.03499200 & 3.28228700 & -1.03249800\end{array}$ $\begin{array}{llll}0.87700500 & 3.18414300 & 1.43811500\end{array}$ $\begin{array}{llll}0.25493600 & 4.44536000 & -1.00422900\end{array}$ $\begin{array}{llll}0.09986700 & 4.34912700 & 1.40333000\end{array}$ $\begin{array}{rrr}-0.20960500 & 4.97337200 & 0.19746600\end{array}$ $\begin{array}{llll}0.01090600 & 4.94533700 & -1.93682100\end{array}$ $\begin{array}{lll}-0.25976100 & 4.77678600 & 2.33463500\end{array}$ $\begin{array}{lll}-0.80889000 & 5.87967500 & 0.19490800\end{array}$ $\begin{array}{lll}2.73115400 & -2.04702000 & 0.15279300\end{array}$ $\begin{array}{llll}2.84564800 & -2.68494900 & -1.09849100\end{array}$ $\begin{array}{llll}2.62392900 & -2.75293000 & 1.36779300\end{array}$ $\begin{array}{llll}2.83843400 & -4.08536600 & -1.10653200\end{array}$ $\begin{array}{lll}2.62259300 & -4.15184200 & 1.29695100\end{array}$ $\begin{array}{lll}2.72722500 & -4.81251700 & 0.07583000\end{array}$ $\begin{array}{lll}2.92747300 & -4.61245700 & -2.05198600\end{array}$ $\begin{array}{lll}2.54208800 & -4.73022000 & 2.21255200\end{array}$ $\begin{array}{llll}2.72913200 & -5.89860400 & 0.04622100\end{array}$ $\begin{array}{lll}1.21328100 & 2.53736800 & 2.77881700\end{array}$ $\begin{array}{lll}1.81807400 & 1.64533500 & 2.58767700\end{array}$ $\begin{array}{lll}2.05620900 & 3.47979800 & 3.66122600\end{array}$ $\begin{array}{lll}2.33868900 & 2.97606800 & 4.59245300\end{array}$ $\begin{array}{llll}2.97457300 & 3.79160900 & 3.15154700\end{array}$ $\begin{array}{lll}1.50058100 & 4.38548400 & 3.92896000\end{array}$ $\begin{array}{lll}-0.05598600 & 2.07115500 & 3.51833300\end{array}$ $\begin{array}{lll}-0.71442700 & 2.91331900 & 3.76046100\end{array}$ $\begin{array}{lll}-0.62595100 & 1.35621700 & 2.91381700\end{array}$ $\begin{array}{lll}0.21299900 & 1.58046500 & 4.46065800\end{array}$ $\begin{array}{lll}2.51945100 & -2.06403300 & 2.72523300\end{array}$ $3.72601500 \quad-2.40509900 \quad 3.62250100$ $\begin{array}{lll}4.67265100 & -2.13173100 & 3.14322300\end{array}$ $\begin{array}{lll}3.65765600 & -1.86377800 & 4.57282000\end{array}$ $3.76653100 \quad-3.47566900 \quad 3.85245900$ $\begin{array}{llll}2.97723300 & -1.92011500 & -2.41257600\end{array}$ $\begin{array}{lll}2.98723900 & -0.84899400 & -2.18756800\end{array}$ $\begin{array}{lll}1.53412600 & 2.73698500 & -2.36706000\end{array}$ $\begin{array}{llll}2.47368600 & 3.73835900 & -3.06842200\end{array}$ $\begin{array}{llll}1.95198700 & 4.66614600 & -3.32859300\end{array}$ $\begin{array}{llll}3.32579600 & 4.00311600 & -2.43252000\end{array}$ $\begin{array}{lll}2.86415000 & 3.30605500 & -3.99662000\end{array}$ $\begin{array}{lll}1.76956600 & -2.17704600 & -3.33550500\end{array}$ $\begin{array}{lll}0.83157600 & -1.88823900 & -2.84746500\end{array}$ $\begin{array}{lll}1.86739200 & -1.59575900 & -4.25957500\end{array}$ $\begin{array}{lll}1.69711800 & -3.23486900 & -3.61386400\end{array}$ $\begin{array}{lll}4.30271800 & -2.24739100 & -3.12863300\end{array}$ $\begin{array}{llll}4.40101000 & -1.64424700 & -4.03819500\end{array}$ $\begin{array}{lll}5.16673400 & -2.03927500 & -2.48794800\end{array}$ $\begin{array}{lll}4.35315500 & -3.30171500 & -3.42266100\end{array}$ $\begin{array}{lll}1.18996500 & -2.40031600 & 3.42901400\end{array}$ $\begin{array}{lll}0.33177100 & -2.11800500 & 2.80869300\end{array}$ $\begin{array}{llll}1.11054700 & -3.47071100 & 3.65029700\end{array}$ $\begin{array}{lll}1.11797300 & -1.85939400 & 4.37944200\end{array}$ $\begin{array}{lll}2.53120500 & -0.98163700 & 2.56315800\end{array}$ $\begin{array}{llll}0.36380800 & 2.33501200 & -3.28591000\end{array}$ $\begin{array}{llll}0.74575300 & 1.90441300 & -4.21867100\end{array}$ $\begin{array}{llll}-0.27994000 & 1.59106100 & -2.80319700\end{array}$ $\begin{array}{lll}-0.25662700 & 3.19918100 & -3.54951300\end{array}$ $\begin{array}{llll}2.11509200 & 1.83000800 & -2.17210400\end{array}$ $\begin{array}{lll}-3.40648800 & -1.93724800 & -2.70589000\end{array}$ $\begin{array}{lll}-5.13336700 & -2.75375800 & -1.57871500\end{array}$ $\begin{array}{lll}-3.54169800 & -3.29325500 & -3.18797700\end{array}$ $\begin{array}{lll}-3.44040000 & -3.26876700 & -4.27256600\end{array}$ $\begin{array}{lll}-2.74811500 & -3.90467500 & -2.74589300\end{array}$ $\begin{array}{lll}-4.93703300 & -3.67595600 & -2.68672700\end{array}$ $\begin{array}{lll}-5.01190100 & -4.69327100 & -2.30147500\end{array}$ $\begin{array}{lll}-5.72920700 & -3.49040500 & -3.41662700\end{array}$ $-4.16669300-1.82743100 \quad-1.60200900$ $\begin{array}{llll}-2.79387100 & -2.19785700 & 0.02956900\end{array}$ 
$\begin{array}{lrrr}\mathrm{H} & -3.71342300 & -2.40128800 & 0.57653600 \\ \mathrm{H} & -2.11460500 & -3.04697500 & -0.03381000\end{array}$

Imaginary Frequency $=-332.6116$

\begin{tabular}{|c|c|c|c|}
\hline Int3f & & & \\
\hline $\mathrm{C}$ & -3.27938500 & -0.00090500 & -0.67743100 \\
\hline $\mathrm{C}$ & -4.43319700 & -0.74094400 & -1.04349100 \\
\hline $\mathrm{C}$ & -5.68346300 & -0.14585900 & -1.05704400 \\
\hline $\mathrm{C}$ & -5.76755400 & 1.21455500 & -0.73211600 \\
\hline $\mathrm{C}$ & -4.62910800 & 1.97129700 & -0.38948400 \\
\hline $\mathrm{C}$ & -3.37857900 & 1.37473900 & -0.35603700 \\
\hline $\mathrm{C}$ & -2.12122000 & -0.82767700 & -0.64981200 \\
\hline $\mathrm{H}$ & -6.57585000 & -0.71200300 & -1.30311500 \\
\hline $\mathrm{H}$ & -6.73859000 & 1.70240500 & -0.74227000 \\
\hline $\mathrm{H}$ & -4.74233200 & 3.02227900 & -0.14340300 \\
\hline $\mathrm{H}$ & -2.49026100 & 1.93452500 & -0.07830800 \\
\hline $\mathrm{C}$ & 1.73589000 & 0.26110700 & 0.13297100 \\
\hline $\mathrm{C}$ & 3.58201200 & 1.49997000 & 0.54035200 \\
\hline $\mathrm{C}$ & 3.96044800 & 0.19402500 & 0.52240200 \\
\hline $\mathrm{H}$ & 4.14843100 & 2.40411500 & 0.69885900 \\
\hline $\mathrm{H}$ & 4.92345000 & -0.27094600 & 0.66333100 \\
\hline $\mathrm{N}$ & 2.81792000 & -0.55061000 & 0.27145100 \\
\hline $\mathrm{N}$ & 2.21639100 & 1.52269400 & 0.30072400 \\
\hline $\mathrm{Au}$ & -0.22063600 & -0.28582500 & -0.25742900 \\
\hline $\mathrm{C}$ & 1.41523700 & 2.72878900 & 0.24238700 \\
\hline $\mathrm{C}$ & 1.24160600 & 3.36075500 & -1.00557700 \\
\hline $\mathrm{C}$ & 0.86005700 & 3.22361100 & 1.43980600 \\
\hline $\mathrm{C}$ & 0.46175100 & 4.52417100 & -1.02936800 \\
\hline $\mathrm{C}$ & 0.09030300 & 4.39087800 & 1.35312300 \\
\hline $\mathrm{C}$ & -0.10952300 & 5.03382200 & 0.13390900 \\
\hline $\mathrm{H}$ & 0.30576700 & 5.04118900 & -1.97157200 \\
\hline $\mathrm{H}$ & -0.35036600 & 4.80534100 & 2.25502600 \\
\hline $\mathrm{H}$ & -0.70478200 & 5.94177500 & 0.09162300 \\
\hline $\mathrm{C}$ & 2.79367100 & -1.99732300 & 0.17605500 \\
\hline $\mathrm{C}$ & 3.01434100 & -2.59054800 & -1.08313100 \\
\hline $\mathrm{C}$ & 2.56893800 & -2.74328600 & 1.35058500 \\
\hline $\mathrm{C}$ & 2.99011100 & -3.98960900 & -1.14235800 \\
\hline $\mathrm{C}$ & 2.55649100 & -4.13840300 & 1.22821400 \\
\hline $\mathrm{C}$ & 2.76222600 & -4.75621900 & -0.00250300 \\
\hline $\mathrm{H}$ & 3.15588000 & -4.48479300 & -2.09440800 \\
\hline $\mathrm{H}$ & 2.38724100 & -4.74817600 & 2.11065200 \\
\hline $\mathrm{H}$ & 2.75170800 & -5.84028200 & -0.07243900 \\
\hline $\mathrm{C}$ & 1.07510800 & 2.55758300 & 2.79630800 \\
\hline $\mathrm{H}$ & 1.68493800 & 1.66090900 & 2.64708000 \\
\hline $\mathrm{C}$ & 1.85097100 & 3.48232400 & 3.75609400 \\
\hline $\mathrm{H}$ & 2.04334300 & 2.96903400 & 4.70481700 \\
\hline $\mathrm{H}$ & 2.81488200 & 3.78410600 & 3.33156300 \\
\hline $\mathrm{H}$ & 1.28642900 & 4.39431000 & 3.98007500 \\
\hline $\mathrm{C}$ & -0.25631500 & 2.09973800 & 3.42301800 \\
\hline $\mathrm{H}$ & -0.92355700 & 2.94726700 & 3.61621700 \\
\hline $\mathrm{H}$ & -0.78266900 & 1.39654300 & 2.76747700 \\
\hline $\mathrm{H}$ & -0.07197200 & 1.59898700 & 4.38006900 \\
\hline $\mathrm{C}$ & 2.35534200 & -2.10070900 & 2.71807300 \\
\hline $\mathrm{C}$ & 3.47825800 & -2.48682500 & 3.70178600 \\
\hline $\mathrm{H}$ & 4.46482600 & -2.21198200 & 3.31242200 \\
\hline $\mathrm{H}$ & 3.33624300 & -1.97491200 & 4.66014400 \\
\hline $\mathrm{H}$ & 3.48722200 & -3.56454000 & 3.89891000 \\
\hline $\mathrm{C}$ & 3.27918300 & -1.78323300 & -2.35077200 \\
\hline $\mathrm{H}$ & 3.26015900 & -0.71957800 & -2.09221900 \\
\hline $\mathrm{C}$ & 1.86665800 & 2.84048200 & -2.29692100 \\
\hline $\mathrm{C}$ & 2.86439800 & 3.85689700 & -2.88712100 \\
\hline $\mathrm{H}$ & 2.36574400 & 4.78791300 & -3.17870400 \\
\hline $\mathrm{H}$ & 3.65063800 & 4.11135500 & -2.16786700 \\
\hline $\mathrm{H}$ & 3.34294700 & 3.44260800 & -3.78149000 \\
\hline $\mathrm{C}$ & 2.18091300 & -2.01259300 & -3.40762700 \\
\hline
\end{tabular}

$\begin{array}{lll}1.19117100 & -1.75178100 & -3.01537000\end{array}$

$\begin{array}{lll}2.37045200 & -1.39396500 & -4.29216700\end{array}$

$\begin{array}{llll}2.14951800 & -3.05794900 & -3.73469200\end{array}$

$\begin{array}{lll}4.67625800 & -2.08606200 & -2.92917700\end{array}$

$\begin{array}{llll}4.87005900 & -1.45451500 & -3.80338000\end{array}$

$\begin{array}{lll}5.46533500 & -1.89809200 & -2.19269800\end{array}$

$\begin{array}{lll}4.76190700 & -3.13050200 & -3.24902800\end{array}$

$\begin{array}{lll}0.96851800 & -2.44577900 & 3.29534900\end{array}$

$\begin{array}{lll}0.16775800 & -2.13523400 & 2.61431700\end{array}$

$\begin{array}{lll}0.86149100 & -3.52184500 & 3.47243000\end{array}$

$\begin{array}{lll}0.81865300 & -1.93600500 & 4.25386800\end{array}$

$\begin{array}{lll}2.39123000 & -1.01314400 & 2.59694300\end{array}$

$\begin{array}{lll}0.79110000 & 2.45384300 & -3.33112500\end{array}$

$\begin{array}{lll}1.26158200 & 2.04213300 & -4.23109700\end{array}$

$\begin{array}{lll}0.10578600 & 1.69885900 & -2.92885100\end{array}$

$\begin{array}{llll}0.19525700 & 3.32139000 & -3.63653200\end{array}$

$\begin{array}{llll}2.43010700 & 1.93134800 & -2.06339300\end{array}$

$-4.10567400 \quad-2.38992400 \quad-2.81090000$

$-4.89841600-3.10205500 \quad-0.78877200$

$-5.23607700 \quad-3.23246100 \quad-3.06419700$

$\begin{array}{lll}-6.14252500 & -2.63344900 & -3.22597300\end{array}$

$\begin{array}{lll}-5.02326500 & -3.81721900 & -3.96153300\end{array}$

$\begin{array}{llll}-5.31642000 & -4.05178600 & -1.78138400\end{array}$

$\begin{array}{lll}-4.63322100 & -4.91054300 & -1.80241000\end{array}$

$\begin{array}{lll}-6.32171300 & -4.39061500 & -1.52264900\end{array}$

$-4.04123500-2.16824400-1.40845800$

$\begin{array}{lll}-2.55483300 & -2.22981600 & -0.95371000\end{array}$

$-2.47249700 \quad-2.82350300 \quad-0.03049300$

$\begin{array}{lll}-1.90856500 & -2.71377500 & -1.69385800\end{array}$

$\begin{array}{lccc}\text { TS3f } & & & \\ \mathrm{C} & -3.36012600 & 0.12162500 & -0.64906700 \\ \mathrm{C} & -4.48238800 & -0.50832600 & -1.20589700 \\ \mathrm{C} & -5.65937000 & 0.18574500 & -1.43635300 \\ \mathrm{C} & -5.69722300 & 1.54772800 & -1.10412900 \\ \mathrm{C} & -4.57997000 & 2.18211200 & -0.55319300 \\ \mathrm{C} & -3.39564300 & 1.47230300 & -0.31601700 \\ \mathrm{C} & -2.22638500 & -0.85054900 & -0.50827800 \\ \mathrm{H} & -6.52889700 & -0.30854400 & -1.85849300 \\ \mathrm{H} & -6.60627500 & 2.11609600 & -1.27687200 \\ \mathrm{H} & -4.63185300 & 3.23762100 & -0.30357600 \\ \mathrm{H} & -2.52840100 & 1.96406400 & 0.11538800 \\ \mathrm{C} & 1.71221900 & 0.21858000 & 0.15105500 \\ \mathrm{C} & 3.55796700 & 1.48178900 & 0.46572400 \\ \mathrm{C} & 3.95255700 & 0.18214500 & 0.43235100 \\ \mathrm{H} & 4.11838000 & 2.39421700 & 0.59516500 \\ \mathrm{H} & 4.92722700 & -0.26994200 & 0.52705500 \\ \mathrm{~N} & 2.80829100 & -0.57987700 & 0.23814000 \\ \mathrm{~N} & 2.18097500 & 1.48664300 & 0.29113400 \\ \mathrm{Au} & -0.23793400 & -0.34131300 & -0.13377300 \\ \mathrm{C} & 1.36202100 & 2.68239000 & 0.27031900 \\ \mathrm{C} & 1.13689800 & 3.32124100 & -0.96573800 \\ \mathrm{C} & 0.84214300 & 3.16044300 & 1.49032600 \\ \mathrm{C} & 0.34252300 & 4.47491700 & -0.95287500 \\ \mathrm{C} & 0.05571800 & 4.31859300 & 1.43984600 \\ \mathrm{C} & -0.19382200 & 4.96849500 & 0.23359200 \\ \mathrm{H} & 0.14496400 & 4.99575400 & -1.88501900 \\ \mathrm{H} & -0.35972600 & 4.71993300 & 2.35945300 \\ \mathrm{H} & -0.80233000 & 5.86843500 & 0.21931900 \\ \mathrm{C} & 2.79914300 & -2.02638700 & 0.14601000 \\ \mathrm{C} & 2.96829800 & -2.61880500 & -1.12156900 \\ \mathrm{C} & 2.64072400 & -2.77379100 & 1.33062000 \\ \mathrm{C} & 2.96002000 & -4.01822700 & -1.17851600 \\ \mathrm{C} & 2.64102800 & -4.16921600 & 1.21023400 \\ \mathrm{C} & 2.79662700 & -4.78615700 & -0.02843800\end{array}$




\begin{tabular}{|c|c|c|c|}
\hline $\mathrm{H}$ & 3.08914000 & -4.51241200 & -2.13680600 \\
\hline $\mathrm{H}$ & 2.52327300 & -4.77990900 & 2.10044800 \\
\hline $\mathrm{H}$ & 2.79890200 & -5.87044000 & -0.09664100 \\
\hline $\mathrm{C}$ & 1.10919400 & 2.48464000 & 2.83237500 \\
\hline $\mathrm{H}$ & 1.72711800 & 1.59859900 & 2.65490400 \\
\hline $\mathrm{C}$ & 1.90030500 & 3.41021300 & 3.77856800 \\
\hline $\mathrm{H}$ & 2.13198800 & 2.88829200 & 4.71374200 \\
\hline $\mathrm{H}$ & 2.84472600 & 3.73365300 & 3.32706900 \\
\hline $\mathrm{H}$ & 1.32823500 & 4.30940000 & 4.03297200 \\
\hline $\mathrm{C}$ & -0.19595400 & 1.99992900 & 3.49331900 \\
\hline $\mathrm{H}$ & -0.86793400 & 2.83508100 & 3.72096000 \\
\hline $\mathrm{H}$ & -0.73408100 & 1.30125000 & 2.84233500 \\
\hline $\mathrm{H}$ & 0.02460700 & 1.48635400 & 4.43591300 \\
\hline $\mathrm{C}$ & 2.48672100 & -2.13190900 & 2.70635700 \\
\hline $\mathrm{C}$ & 3.66428700 & -2.49920600 & 3.63174200 \\
\hline $\mathrm{H}$ & 4.62514900 & -2.20915100 & 3.19243000 \\
\hline $\mathrm{H}$ & 3.56351400 & -1.98831300 & 4.59582200 \\
\hline $\mathrm{H}$ & 3.70028400 & -3.57644700 & 3.82854000 \\
\hline $\mathrm{C}$ & 3.16374100 & -1.80927100 & -2.40044200 \\
\hline $\mathrm{H}$ & 3.14687700 & -0.74600200 & -2.14051500 \\
\hline $\mathrm{C}$ & 1.71683200 & 2.81521100 & -2.28334900 \\
\hline $\mathrm{C}$ & 2.66811200 & 3.85100000 & -2.91517200 \\
\hline $\mathrm{H}$ & 2.13947100 & 4.77171600 & -3.18566900 \\
\hline $\mathrm{H}$ & 3.47918700 & 4.12180200 & -2.23019800 \\
\hline $\mathrm{H}$ & 3.11634500 & 3.44557600 & -3.82912900 \\
\hline $\mathrm{C}$ & 2.01768000 & -2.04874300 & -3.40299400 \\
\hline $\mathrm{H}$ & 1.04647700 & -1.79074900 & -2.96511800 \\
\hline $\mathrm{H}$ & 2.16179300 & -1.43251300 & -4.29759500 \\
\hline $\mathrm{H}$ & 1.97682100 & -3.09562500 & -3.72448700 \\
\hline $\mathrm{C}$ & 4.53393600 & -2.09850100 & -3.04602000 \\
\hline $\mathrm{H}$ & 4.67811700 & -1.46473700 & -3.92811500 \\
\hline $\mathrm{H}$ & 5.35598900 & -1.90257000 & -2.34876000 \\
\hline $\mathrm{H}$ & 4.61425300 & -3.14193900 & -3.37045600 \\
\hline $\mathrm{C}$ & 1.13707000 & -2.49681200 & 3.35518100 \\
\hline $\mathrm{H}$ & 0.29749000 & -2.19585100 & 2.71768300 \\
\hline $\mathrm{H}$ & 1.05432200 & -3.57431400 & 3.53659000 \\
\hline $\mathrm{H}$ & 1.03079000 & -1.98939600 & 4.32060500 \\
\hline $\mathrm{H}$ & 2.49930900 & -1.04432800 & 2.58219400 \\
\hline $\mathrm{C}$ & 0.60437500 & 2.40783100 & -3.26937900 \\
\hline $\mathrm{H}$ & 1.04216700 & 2.00536900 & -4.18984300 \\
\hline $\mathrm{H}$ & -0.04776400 & 1.64026600 & -2.83691200 \\
\hline $\mathrm{H}$ & -0.02147000 & 3.26358100 & -3.54651700 \\
\hline $\mathrm{H}$ & 2.30816400 & 1.91736500 & -2.07654300 \\
\hline $\mathrm{O}$ & -4.13464500 & -2.31537800 & -2.84845100 \\
\hline $\mathrm{O}$ & -5.06683800 & -2.84101900 & -0.83351500 \\
\hline $\mathrm{C}$ & -4.88850500 & -3.53018200 & -3.03374400 \\
\hline $\mathrm{H}$ & -5.83990800 & -3.28443300 & -3.51746800 \\
\hline $\mathrm{H}$ & -4.31736300 & -4.20676400 & -3.67394800 \\
\hline $\mathrm{C}$ & -5.08381800 & -4.04916100 & -1.60833000 \\
\hline $\mathrm{H}$ & -4.27206300 & -4.72097500 & -1.29810500 \\
\hline $\mathrm{H}$ & -6.04271500 & -4.54013400 & -1.43452000 \\
\hline $\mathrm{C}$ & -4.17756000 & -1.96807200 & -1.47240800 \\
\hline $\mathrm{C}$ & -2.72582900 & -2.07713400 & -0.95736500 \\
\hline $\mathrm{H}$ & -2.39203000 & -1.63276200 & 0.41686700 \\
\hline $\mathrm{H}$ & -2.16520900 & -3.00809400 & -0.96347100 \\
\hline
\end{tabular}

$\begin{array}{lrrr}\text { Int4f } & & & \\ \mathrm{C} & -2.80898500 & -0.22918600 & -3.02891400 \\ \mathrm{C} & -3.49438500 & -0.19462700 & -1.80239500 \\ \mathrm{C} & -4.36126500 & 0.84206400 & -1.50501100 \\ \mathrm{C} & -4.55653000 & 1.84301900 & -2.47083700 \\ \mathrm{C} & -3.89002100 & 1.79912300 & -3.69893400 \\ \mathrm{C} & -2.99826200 & 0.76001300 & -3.99078700 \\ \mathrm{C} & -1.95030800 & -1.42780600 & -3.04851300\end{array}$

$\begin{array}{lll}-4.88416400 & 0.88256700 & -0.55429700\end{array}$ $\begin{array}{llll}-5.24250600 & 2.65916500 & -2.26365000\end{array}$ $\begin{array}{lll}-4.06401600 & 2.58024600 & -4.43275300\end{array}$ $\begin{array}{lll}-2.47355100 & 0.72938100 & -4.94165500\end{array}$ $\begin{array}{lll}1.75824700 & -0.33000400 & -0.99334200\end{array}$ $\begin{array}{llll}3.46704000 & 1.01895000 & -0.41802100\end{array}$ $\begin{array}{lll}3.88554900 & -0.26414400 & -0.25495300\end{array}$ $\begin{array}{llll}3.97005700 & 1.95930900 & -0.25705300\end{array}$ $\begin{array}{lll}4.82661000 & -0.67068800 & 0.08041300\end{array}$ $\begin{array}{lll}2.82361900 & -1.08113100 & -0.61275400\end{array}$ $\begin{array}{lll}2.15704600 & 0.96161700 & -0.87031800\end{array}$ $\begin{array}{llll}-0.04811400 & -1.02899300 & -1.61778600\end{array}$ $\begin{array}{lll}1.34915700 & 2.12969700 & -1.16732700\end{array}$ $\begin{array}{lll}1.39528400 & 2.66123300 & -2.47166300\end{array}$ $\begin{array}{llll}0.58401200 & 2.69614300 & -0.12807400\end{array}$ $\begin{array}{lll}0.62565600 & 3.80406700 & -2.72138400\end{array}$ $\begin{array}{llll}-0.16289300 & 3.83927100 & -0.43922500\end{array}$ $\begin{array}{llll}-0.14472500 & 4.38739000 & -1.71898600\end{array}$ $\begin{array}{llll}0.63589900 & 4.24594500 & -3.71322500\end{array}$ $\begin{array}{lll}-0.76170300 & 4.30970400 & 0.33462200\end{array}$ $\begin{array}{lll}-0.72919900 & 5.27736000 & -1.93450600\end{array}$ $\begin{array}{llll}2.85131500 & -2.53071400 & -0.56534200\end{array}$ $\begin{array}{lll}3.26487900 & -3.23344300 & -1.71477300\end{array}$ $\begin{array}{lll}2.47804800 & -3.16671500 & 0.63597900\end{array}$ $3.27664000 \quad-4.63178900-1.63652500$ $\begin{array}{llll}2.50934100 & -4.56672600 & 0.65214000\end{array}$ $\begin{array}{llll}2.90035700 & -5.29258500 & -0.47007100\end{array}$ $\begin{array}{lll}3.58938400 & -5.21027200 & -2.50053600\end{array}$ $\begin{array}{llll}2.22943200 & -5.09448700 & 1.55887500\end{array}$ $\begin{array}{lll}2.91975300 & -6.37805000 & -0.43290600\end{array}$ $\begin{array}{lll}0.57034400 & 2.14321600 & 1.29422400\end{array}$ $\begin{array}{lll}1.14003100 & 1.20793900 & 1.30495700\end{array}$ $\begin{array}{lll}1.26618700 & 3.11618000 & 2.26851200\end{array}$ $\begin{array}{lll}1.29720100 & 2.68817300 & 3.27676700\end{array}$ $\begin{array}{llll}2.29523900 & 3.33105500 & 1.95900000\end{array}$ $\begin{array}{lll}0.73128600 & 4.07072400 & 2.32732900\end{array}$ $\begin{array}{lll}-0.85515700 & 1.80616200 & 1.77196500\end{array}$ $\begin{array}{lll}-1.48742700 & 2.70005100 & 1.81980900\end{array}$ $\begin{array}{lll}-1.34206100 & 1.07952500 & 1.11333400\end{array}$ $\begin{array}{lll}-0.81690800 & 1.37950600 & 2.78106000\end{array}$ $\begin{array}{lll}2.06545300 & -2.40305600 & 1.89164900\end{array}$ $3.01544300 \quad-2.70164800 \quad 3.06901200$ $\begin{array}{lll}4.05635900 & -2.47772500 & 2.81114700\end{array}$ $\begin{array}{llll}2.74251500 & -2.09426100 & 3.93908500\end{array}$ $\begin{array}{lll}2.96676400 & -3.75371900 & 3.37081100\end{array}$ $\begin{array}{lll}3.70149000 & -2.54275900 & -3.00374000\end{array}$ $\begin{array}{lll}3.60783700 & -1.46085000 & -2.86395800\end{array}$ $\begin{array}{llll}2.24169700 & 2.05871900 & -3.58944700\end{array}$ $\begin{array}{llll}3.33248100 & 3.04084100 & -4.06196900\end{array}$ $\begin{array}{llll}2.89677600 & 3.94695600 & -4.49720800\end{array}$ $\begin{array}{lll}3.98094300 & 3.34863100 & -3.23425200\end{array}$ $\begin{array}{lll}3.95930100 & 2.57170400 & -4.82843900\end{array}$ $\begin{array}{lll}2.79328500 & -2.92540000 & -4.18870700\end{array}$ $\begin{array}{lll}1.74579700 & -2.67739000 & -3.98043600\end{array}$ $\begin{array}{llll}3.09990200 & -2.38481800 & -5.09126000\end{array}$ $\begin{array}{lll}2.84707700 & -3.99713000 & -4.40960500\end{array}$ $\begin{array}{lll}5.18145100 & -2.83586600 & -3.32245800\end{array}$ $\begin{array}{llll}5.49215200 & -2.28926200 & -4.21978000\end{array}$ $\begin{array}{lll}5.83692300 & -2.53557200 & -2.49758300\end{array}$ $\begin{array}{llll}5.34844300 & -3.90256200 & -3.50827200\end{array}$ $\begin{array}{lll}0.60041100 & -2.69086600 & 2.27455200\end{array}$ $\begin{array}{lll}-0.08438000 & -2.40295200 & 1.46857700\end{array}$ $\begin{array}{lll}0.44420900 & -3.75366400 & 2.49179100\end{array}$ $\begin{array}{lll}0.32621900 & -2.12384700 & 3.17152100\end{array}$ $\begin{array}{lll}2.13842400 & -1.33057900 & 1.68333900\end{array}$ $\begin{array}{lll}1.36812900 & 1.59002000 & -4.76936400\end{array}$ $\begin{array}{lll}1.98991200 & 1.11840800 & -5.53877200\end{array}$ 


$\begin{array}{lrrr}\mathrm{H} & 0.61864500 & 0.86113700 & -4.43945100 \\ \mathrm{H} & 0.83941200 & 2.42784000 & -5.23747000 \\ \mathrm{H} & 2.75204400 & 1.17291200 & -3.19756800 \\ \mathrm{O} & -4.21334100 & -2.35775700 & -0.86899500 \\ \mathrm{O} & -2.74221300 & -1.09240800 & 0.33664200 \\ \mathrm{C} & -4.61279800 & -2.41877600 & 0.50563700 \\ \mathrm{H} & -5.41609100 & -1.69778900 & 0.70560900 \\ \mathrm{H} & -4.97108200 & -3.43014400 & 0.70931200 \\ \mathrm{C} & -3.32639200 & -2.05037000 & 1.23332500 \\ \mathrm{H} & -2.66418400 & -2.91694400 & 1.35840100 \\ \mathrm{H} & -3.47405700 & -1.55913700 & 2.19701200 \\ \mathrm{C} & -3.14065100 & -1.43245900 & -0.97774200 \\ \mathrm{H} & -1.45047500 & -1.79917200 & -3.93887600 \\ \mathrm{C} & -2.05520600 & -2.10852300 & -1.84993200 \\ \mathrm{H} & -1.78004900 & -3.14701600 & -1.69391700\end{array}$

\begin{tabular}{|c|c|c|c|}
\hline $1 f^{\prime}$ & & & \\
\hline $\mathrm{C}$ & -3.12598600 & -1.07944100 & -0.31433400 \\
\hline $\mathrm{C}$ & -4.48990400 & -1.15023200 & -0.61022400 \\
\hline $\mathrm{C}$ & -5.29869900 & -0.02043100 & -0.62570700 \\
\hline $\mathrm{C}$ & -4.70895700 & 1.21103500 & -0.32893500 \\
\hline $\mathrm{C}$ & -3.34259800 & 1.29069600 & -0.02815500 \\
\hline $\mathrm{C}$ & -2.53392600 & 0.15426800 & -0.01456700 \\
\hline $\mathrm{C}$ & -2.48482400 & -2.38071600 & -0.36530600 \\
\hline $\mathrm{C}$ & -1.43811100 & -3.12725400 & -0.25385600 \\
\hline $\mathrm{H}$ & -6.35629500 & -0.09143100 & -0.85880500 \\
\hline $\mathrm{H}$ & -5.31454900 & 2.11203700 & -0.33154200 \\
\hline $\mathrm{H}$ & -2.89703000 & 2.25452000 & 0.19857600 \\
\hline $\mathrm{H}$ & -1.47658800 & 0.22303600 & 0.21739900 \\
\hline $\mathrm{C}$ & 2.27542600 & -1.58122000 & 0.72238100 \\
\hline $\mathrm{C}$ & 4.01944100 & -0.25322900 & 1.27105800 \\
\hline $\mathrm{C}$ & 4.48036000 & -1.52920900 & 1.21102600 \\
\hline $\mathrm{H}$ & 4.51800000 & 0.67611600 & 1.49678300 \\
\hline $\mathrm{H}$ & 5.46381600 & -1.94097900 & 1.37384300 \\
\hline $\mathrm{N}$ & 3.39842000 & -2.33150000 & 0.87367600 \\
\hline $\mathrm{N}$ & 2.66413800 & -0.30211400 & 0.96866900 \\
\hline $\mathrm{Au}$ & 0.40942100 & -2.27324800 & 0.23480400 \\
\hline $\mathrm{C}$ & 1.80202100 & 0.86108900 & 0.92663100 \\
\hline $\mathrm{C}$ & 1.67896500 & 1.56140300 & -0.29074700 \\
\hline $\mathrm{C}$ & 1.14997800 & 1.25709100 & 2.11214400 \\
\hline $\mathrm{C}$ & 0.86072900 & 2.69859400 & -0.29401200 \\
\hline $\mathrm{C}$ & 0.34495300 & 2.40195700 & 2.04739600 \\
\hline $\mathrm{C}$ & 0.20331400 & 3.11748000 & 0.86053300 \\
\hline $\mathrm{H}$ & 0.74492900 & 3.26862900 & -1.21107400 \\
\hline $\mathrm{H}$ & -0.16810100 & 2.74215000 & 2.94197200 \\
\hline $\mathrm{H}$ & -0.41271300 & 4.01256500 & 0.83802000 \\
\hline $\mathrm{C}$ & 3.46994800 & -3.76955600 & 0.70746700 \\
\hline $\mathrm{C}$ & 3.76810000 & -4.28331600 & -0.57058100 \\
\hline $\mathrm{C}$ & 3.25745300 & -4.58692800 & 1.83580800 \\
\hline $\mathrm{C}$ & 3.83980100 & -5.67614500 & -0.69809700 \\
\hline $\mathrm{C}$ & 3.34128200 & -5.97193500 & 1.64581200 \\
\hline $\mathrm{C}$ & 3.62740800 & -6.51230100 & 0.39484800 \\
\hline $\mathrm{H}$ & 4.06885000 & -6.11060700 & -1.66669700 \\
\hline $\mathrm{H}$ & 3.18288600 & -6.63525900 & 2.49094600 \\
\hline $\mathrm{H}$ & 3.69072900 & -7.58989700 & 0.27231900 \\
\hline $\mathrm{C}$ & 1.29538000 & 0.50667300 & 3.43290000 \\
\hline $\mathrm{H}$ & 1.96131900 & -0.34672000 & 3.27130000 \\
\hline $\mathrm{C}$ & 1.94410200 & 1.39261600 & 4.51523700 \\
\hline $\mathrm{H}$ & 2.08914200 & 0.81879900 & 5.43732000 \\
\hline $\mathrm{H}$ & 2.92089200 & 1.76995500 & 4.19292900 \\
\hline $\mathrm{H}$ & 1.31682600 & 2.25767600 & 4.75807600 \\
\hline $\mathrm{C}$ & -0.05580400 & -0.05843800 & 3.91321500 \\
\hline $\mathrm{H}$ & -0.77533000 & 0.74103200 & 4.12409400 \\
\hline $\mathrm{H}$ & -0.49811000 & -0.72287100 & 3.16226300 \\
\hline $\mathrm{H}$ & 0.08135200 & -0.63230700 & 4.83662400 \\
\hline
\end{tabular}

$\begin{array}{crrr}\mathrm{C} & 2.95268000 & -4.02987200 & 3.22368100 \\ \mathrm{C} & 4.05412700 & -4.40570400 & 4.23507800 \\ \mathrm{H} & 5.03888400 & -4.05761200 & 3.90406300 \\ \mathrm{H} & 3.84377700 & -3.95333700 & 5.21073200 \\ \mathrm{H} & 4.11606300 & -5.49013900 & 4.37850100 \\ \mathrm{C} & 4.01886000 & -3.39733100 & -1.78756700 \\ \mathrm{H} & 3.90650300 & -2.35228300 & -1.48125100 \\ \mathrm{C} & 2.39698300 & 1.13946000 & -1.56928900 \\ \mathrm{C} & 3.39993900 & 2.21528100 & -2.03210200 \\ \mathrm{H} & 2.89431000 & 3.15162500 & -2.29328400 \\ \mathrm{H} & 4.13583300 & 2.44075500 & -1.25228800 \\ \mathrm{H} & 3.94095900 & 1.87124100 & -2.92057300 \\ \mathrm{C} & 2.98311100 & -3.65640800 & -2.89909300 \\ \mathrm{H} & 1.96206600 & -3.49597300 & -2.53458300 \\ \mathrm{H} & 3.15506200 & -2.97830300 & -3.74277600 \\ \mathrm{H} & 3.04841500 & -4.68185400 & -3.27973700 \\ \mathrm{C} & 5.45702500 & -3.56543400 & -2.31741400 \\ \mathrm{H} & 5.63344800 & -2.88688600 & -3.15953000 \\ \mathrm{H} & 6.19893900 & -3.34458000 & -1.54195800 \\ \mathrm{H} & 5.63857100 & -4.58688100 & -2.66977400 \\ \mathrm{C} & 1.56485300 & -4.48004000 & 3.72039500 \\ \mathrm{H} & 0.77549900 & -4.16698400 & 3.02753000 \\ \mathrm{H} & 1.50903300 & -5.56933300 & 3.82575500 \\ \mathrm{H} & 1.35260800 & -4.04034600 & 4.70160900 \\ \mathrm{H} & 2.93222000 & -2.93715900 & 3.15922100 \\ \mathrm{C} & 1.39899200 & 0.79060900 & -2.69083300 \\ \mathrm{H} & 1.93714700 & 0.44792200 & -3.58177100 \\ \mathrm{H} & 0.71357000 & -0.00580500 & -2.37973500 \\ \mathrm{H} & 0.79777800 & 1.65952700 & -2.98150700 \\ \mathrm{H} & 2.97050800 & 0.23177500 & -1.35637800 \\ \mathrm{O} & -5.28708000 & -2.87567500 & -2.15472200 \\ \mathrm{O} & -5.72398700 & -3.15292500 & 0.02203200 \\ \mathrm{C} & -6.29769900 & -3.91269000 & -2.09975700 \\ \mathrm{H} & -7.24007700 & -3.47668000 & -2.44285500 \\ \mathrm{H} & -6.00336200 & -4.72581600 & -2.76587000 \\ \mathrm{C} & -6.31545600 & -4.30546900 & -0.61704400 \\ \mathrm{H} & -5.70140700 & -5.18786100 & -0.40537200 \\ \mathrm{H} & -7.31266600 & -4.44021300 & -0.19755300 \\ \mathrm{C} & -4.89398400 & -2.57233200 & -0.88570300 \\ \mathrm{H} & -3.71162000 & -3.06604100 & -0.72149500 \\ \mathrm{H} & -1.41889800 & -4.20427000 & -0.39048100 \\ \mathrm{Imaginary} & \mathrm{Frequency}=-765.5703 & \\ & & & \\ \mathrm{H} & & & \\ \mathrm{H} & & \end{array}$

$\begin{array}{crrr}\text { Int2f' } & & & \\ \mathrm{C} & -4.37279500 & -0.50001700 & -1.35770800 \\ \mathrm{C} & -5.25865400 & -1.60910600 & -1.57520400 \\ \mathrm{C} & -6.34279200 & -1.50617600 & -2.49607900 \\ \mathrm{C} & -6.62278500 & -0.30492900 & -3.10992500 \\ \mathrm{C} & -5.83370600 & 0.82209800 & -2.80720700 \\ \mathrm{C} & -4.74874700 & 0.72392500 & -1.94892000 \\ \mathrm{H} & -6.97733600 & -2.36895700 & -2.66505800 \\ \mathrm{H} & -7.45903600 & -0.22268700 & -3.79601000 \\ \mathrm{H} & -6.06454700 & 1.77783000 & -3.26931700 \\ \mathrm{H} & -4.12094200 & 1.59211300 & -1.77173500 \\ \mathrm{C} & 1.52970200 & -1.85400200 & 0.93211500 \\ \mathrm{C} & 3.48696300 & -1.29449400 & 1.93082900 \\ \mathrm{C} & 3.55583100 & -2.61235800 & 1.61310800 \\ \mathrm{H} & 4.19562200 & -0.63820000 & 2.41081400 \\ \mathrm{H} & 4.33692900 & -3.34179200 & 1.75874800 \\ \mathrm{~N} & 2.35027300 & -2.93922100 & 1.00369100 \\ \mathrm{~N} & 2.24212300 & -0.84604300 & 1.50758700 \\ \mathrm{Au} & -0.37027500 & -1.74239600 & 0.10809300 \\ \mathrm{C} & 1.78393900 & 0.51938200 & 1.65856400 \\ \mathrm{C} & 2.06827100 & 1.44128800 & 0.63142300 \\ \mathrm{C} & 1.09532800 & 0.87276200 & 2.83585500\end{array}$




\begin{tabular}{|c|c|c|c|}
\hline $\mathrm{C}$ & 1.62927600 & 2.75880500 & 0.81279100 \\
\hline $\mathrm{C}$ & 0.68176400 & 2.20471700 & 2.96226400 \\
\hline $\mathrm{C}$ & 0.94375500 & 3.13868500 & 1.96348500 \\
\hline $\mathrm{H}$ & 1.83033900 & 3.49702600 & 0.04205900 \\
\hline $\mathrm{H}$ & 0.14957700 & 2.51401200 & 3.85702600 \\
\hline $\mathrm{H}$ & 0.61628000 & 4.16759800 & 2.08439700 \\
\hline $\mathrm{C}$ & 2.03197400 & -4.26236400 & 0.51123200 \\
\hline $\mathrm{C}$ & 2.33975200 & -4.57563000 & -0.82780100 \\
\hline $\mathrm{C}$ & 1.45323000 & -5.18971500 & 1.40039300 \\
\hline $\mathrm{C}$ & 2.03794400 & -5.87014900 & -1.26942700 \\
\hline $\mathrm{C}$ & 1.17614500 & -6.46941500 & 0.90391800 \\
\hline $\mathrm{C}$ & 1.46372000 & -6.80817800 & -0.41592700 \\
\hline $\mathrm{H}$ & 2.26244900 & -6.14709200 & -2.29527100 \\
\hline $\mathrm{H}$ & 0.73533800 & -7.21220900 & 1.56251700 \\
\hline $\mathrm{H}$ & 1.24597600 & -7.80898400 & -0.77884600 \\
\hline $\mathrm{C}$ & 0.79594300 & -0.12240100 & 3.95298200 \\
\hline $\mathrm{H}$ & 1.18637200 & -1.10018500 & 3.65390100 \\
\hline $\mathrm{C}$ & 1.50446000 & 0.27117200 & 5.26428300 \\
\hline $\mathrm{H}$ & 1.31817800 & -0.48200200 & 6.03843200 \\
\hline $\mathrm{H}$ & 2.58815800 & 0.35395300 & 5.12509400 \\
\hline $\mathrm{H}$ & 1.14400500 & 1.23383900 & 5.64425000 \\
\hline $\mathrm{C}$ & -0.72105600 & -0.28978300 & 4.16713400 \\
\hline $\mathrm{H}$ & -1.18785600 & 0.64687800 & 4.49271300 \\
\hline $\mathrm{H}$ & -1.21476400 & -0.61154700 & 3.24334000 \\
\hline $\mathrm{H}$ & -0.91333400 & -1.04227500 & 4.94097600 \\
\hline $\mathrm{C}$ & 1.12732800 & -4.85243700 & 2.85259900 \\
\hline $\mathrm{C}$ & 1.91883500 & -5.73984500 & 3.83340600 \\
\hline $\mathrm{H}$ & 2.99856400 & -5.65131500 & 3.67026600 \\
\hline $\mathrm{H}$ & 1.70709200 & -5.44670300 & 4.86793800 \\
\hline $\mathrm{H}$ & 1.65273000 & -6.79752700 & 3.72657400 \\
\hline $\mathrm{C}$ & 2.97565700 & -3.57636800 & -1.78957900 \\
\hline $\mathrm{H}$ & 3.15143400 & -2.64274500 & -1.24646300 \\
\hline $\mathrm{C}$ & 2.81743400 & 1.06123800 & -0.64212600 \\
\hline $\mathrm{C}$ & 4.12794600 & 1.85971600 & -0.78854200 \\
\hline $\mathrm{H}$ & 3.93659200 & 2.93341800 & -0.89495500 \\
\hline $\mathrm{H}$ & 4.78078400 & 1.72270200 & 0.08076900 \\
\hline $\mathrm{H}$ & 4.67515900 & 1.53115600 & -1.67936600 \\
\hline $\mathrm{C}$ & 2.03087900 & -3.24618500 & -2.96229900 \\
\hline $\mathrm{H}$ & 1.07885600 & -2.84180500 & -2.60024800 \\
\hline $\mathrm{H}$ & 2.49122700 & -2.50013700 & -3.62025000 \\
\hline $\mathrm{H}$ & 1.81565500 & -4.13480000 & -3.56698800 \\
\hline $\mathrm{C}$ & 4.34305300 & -4.07361200 & -2.29908700 \\
\hline $\mathrm{H}$ & 4.80648400 & -3.31567000 & -2.94061200 \\
\hline $\mathrm{H}$ & 5.02886400 & -4.28274000 & -1.47054300 \\
\hline $\mathrm{H}$ & 4.24404500 & -4.99141500 & -2.88958900 \\
\hline $\mathrm{C}$ & -0.38794500 & -4.93940700 & 3.12190100 \\
\hline $\mathrm{H}$ & -0.94118700 & -4.26861000 & 2.45438200 \\
\hline $\mathrm{H}$ & -0.76218300 & -5.95931300 & 2.97357600 \\
\hline $\mathrm{H}$ & -0.60744300 & -4.65158100 & 4.15661600 \\
\hline $\mathrm{H}$ & 1.42591700 & -3.81633000 & 3.03855700 \\
\hline $\mathrm{C}$ & 1.92357800 & 1.22376500 & -1.88752800 \\
\hline $\mathrm{H}$ & 2.46416000 & 0.90573200 & -2.78654300 \\
\hline $\mathrm{H}$ & 1.01438900 & 0.61823800 & -1.80202900 \\
\hline $\mathrm{H}$ & 1.62346100 & 2.26797900 & -2.03233200 \\
\hline $\mathrm{H}$ & 3.08858900 & 0.00281500 & -0.57759300 \\
\hline $\mathrm{O}$ & -5.82177100 & -3.89807000 & -1.19992000 \\
\hline $\mathrm{O}$ & -4.70186500 & -2.89572700 & 0.40211200 \\
\hline $\mathrm{C}$ & -5.83047000 & -4.86714700 & -0.10735300 \\
\hline $\mathrm{H}$ & -6.84473000 & -4.89790800 & 0.29705700 \\
\hline $\mathrm{H}$ & -5.55835700 & -5.83488400 & -0.52708900 \\
\hline $\mathrm{C}$ & -4.79593100 & -4.27697000 & 0.85625300 \\
\hline $\mathrm{H}$ & -3.80041300 & -4.71815000 & 0.76505300 \\
\hline $\mathrm{H}$ & -5.10932700 & -4.25681300 & 1.89925200 \\
\hline $\mathrm{C}$ & -5.21571300 & -2.79877600 & -0.79858200 \\
\hline $\mathrm{C}$ & -2.23633200 & -1.63296600 & -0.70123800 \\
\hline $\mathrm{H}$ & -2.59091500 & -2.49437800 & -1.28066800 \\
\hline & -308899000 & -0.575627 & -0.669 \\
\hline
\end{tabular}

$\begin{array}{lrrr}\text { TS2f' } & & & \\ \mathrm{C} & -4.69545900 & -0.38240300 & -0.74257500 \\ \mathrm{C} & -4.83138500 & -1.60358700 & -1.43554700 \\ \mathrm{C} & -5.96875300 & -1.88525100 & -2.18329900 \\ \mathrm{C} & -7.01718700 & -0.96052700 & -2.14907600 \\ \mathrm{C} & -6.92181700 & 0.22743100 & -1.40150900 \\ \mathrm{C} & -5.76909500 & 0.52521500 & -0.68433200 \\ \mathrm{H} & -6.06181300 & -2.81337600 & -2.73756800 \\ \mathrm{H} & -7.93636700 & -1.17769900 & -2.68512300 \\ \mathrm{H} & -7.75695100 & 0.92096900 & -1.39486100 \\ \mathrm{H} & -5.67670200 & 1.45754800 & -0.13526700 \\ \mathrm{C} & 1.30313600 & -1.80447900 & 0.93658700 \\ \mathrm{C} & 3.32182900 & -1.45614600 & 1.90184800 \\ \mathrm{C} & 3.22229700 & -2.78399100 & 1.63785400 \\ \mathrm{H} & 4.11155300 & -0.87602000 & 2.35263600 \\ \mathrm{H} & 3.90638000 & -3.59971100 & 1.81091000 \\ \mathrm{~N} & 1.97911800 & -2.98081100 & 1.04769600 \\ \mathrm{~N} & 2.13874000 & -0.87157600 & 1.46753900 \\ \mathrm{Au} & -0.55518100 & -1.46511200 & 0.09064200 \\ \mathrm{C} & 1.84103400 & 0.54184700 & 1.58283900 \\ \mathrm{C} & 2.20542900 & 1.39602800 & 0.52312500 \\ \mathrm{C} & 1.21567500 & 1.00124300 & 2.75959500 \\ \mathrm{C} & 1.90873300 & 2.75721000 & 0.66753200 \\ \mathrm{C} & 0.94411000 & 2.37230900 & 2.84688400 \\ \mathrm{C} & 1.28372400 & 3.24170500 & 1.81348300 \\ \mathrm{C} & 2.17543500 & 3.44707800 & -0.12760300 \\ \mathrm{H} & 4.30748100 & 1.52411700 & -0.88603500 \\ \mathrm{H} & 4.93220100 & 1.31285900 & -0.01102700 \\ \mathrm{H} & 4.81232800 & 1.11883200 & -1.77014700 \\ \mathrm{H} & 1.60688500 & -3.49614500 & -2.93101400 \\ \mathrm{H} & 0.69887600 & -2.96272500 & -2.62697800 \\ \mathrm{H} & 2.16440500 & -2.85181500 & -3.61996600 \\ \mathrm{H} & 1.30822100 & -4.39218400 & -3.48765000 \\ \mathrm{H} & & & \\ \mathrm{H} & & & \end{array}$




$\begin{array}{lrrr}\mathrm{C} & 3.78254800 & -4.54913000 & -2.14258200 \\ \mathrm{H} & 4.34670200 & -3.90719800 & -2.82827100 \\ \mathrm{H} & 4.42296600 & -4.77249000 & -1.28224500 \\ \mathrm{H} & 3.58059800 & -5.49296400 & -2.66148100 \\ \mathrm{C} & -0.93462400 & -4.48591100 & 3.34590900 \\ \mathrm{H} & -1.42220400 & -3.76357600 & 2.68131600 \\ \mathrm{H} & -1.45726000 & -5.44496700 & 3.25173300 \\ \mathrm{H} & -1.06720100 & -4.13930900 & 4.37694800 \\ \mathrm{H} & 1.01246500 & -3.63197000 & 3.14922600 \\ \mathrm{C} & 2.04910000 & 1.16262100 & -1.99715500 \\ \mathrm{H} & 2.54932200 & 0.75998500 & -2.88545200 \\ \mathrm{H} & 1.06576000 & 0.68644900 & -1.91177600 \\ \mathrm{H} & 1.89195500 & 2.23436900 & -2.16311900 \\ \mathrm{H} & 3.03510000 & -0.18055400 & -0.65943800 \\ \mathrm{O} & -3.21481900 & -3.18413700 & -2.31957500 \\ \mathrm{O} & -3.69343600 & -3.30334400 & -0.14016900 \\ \mathrm{C} & -2.79747100 & -4.50927400 & -1.90783100 \\ \mathrm{H} & -3.52610500 & -5.22779000 & -2.29492900 \\ \mathrm{H} & -1.81267600 & -4.71023400 & -2.33150500 \\ \mathrm{C} & -2.79679000 & -4.40876900 & -0.38029800 \\ \mathrm{H} & -1.80931800 & -4.17337700 & 0.02594700 \\ \mathrm{H} & -3.21522200 & -5.27967600 & 0.12511500 \\ \mathrm{C} & -3.64869600 & -2.49013300 & -1.23460100 \\ \mathrm{C} & -2.38061600 & -1.07540600 & -0.80220700 \\ \mathrm{H} & -2.29859300 & -0.98137200 & -1.88833200 \\ \mathrm{C} & -3.36245800 & -0.24385500 & -0.23491200 \\ \mathrm{H} & -3.16746700 & 0.39157800 & 0.62889100 \\ \mathrm{Imaginary} & \text { Frequency }=-436.3658 & \\ & & & \end{array}$

$\begin{array}{crrr}\text { Int3f' } & & & \\ \mathrm{C} & -3.11623800 & -2.96865700 & -1.03323700 \\ \mathrm{C} & -3.63512700 & -3.24081600 & -2.31022000 \\ \mathrm{C} & -4.58767300 & -2.41417400 & -2.87950900 \\ \mathrm{C} & -5.02408900 & -1.29943700 & -2.14533200 \\ \mathrm{C} & -4.51299200 & -1.03086700 & -0.87205700 \\ \mathrm{C} & -3.54695500 & -1.86659100 & -0.29888300 \\ \mathrm{C} & -2.14520800 & -4.02482800 & -0.68924000 \\ \mathrm{H} & -5.00113700 & -2.62469200 & -3.86119300 \\ \mathrm{H} & -5.77882600 & -0.64285800 & -2.56816900 \\ \mathrm{H} & -4.87266400 & -0.16780500 & -0.32008800 \\ \mathrm{H} & -3.15145300 & -1.65976300 & 0.69169400 \\ \mathrm{C} & 1.69520200 & -2.83346600 & -2.41643900 \\ \mathrm{C} & 3.35642600 & -1.42126400 & -2.97775200 \\ \mathrm{C} & 3.88142000 & -2.67465800 & -2.93567100 \\ \mathrm{H} & 3.80616000 & -0.46680100 & -3.20143000 \\ \mathrm{H} & 4.88108700 & -3.03630800 & -3.11802400 \\ \mathrm{~N} & 2.84723800 & -3.53108400 & -2.58905400 \\ \mathrm{~N} & 2.01133300 & -1.53627800 & -2.65790900 \\ \mathrm{Au} & -0.10882200 & -3.62167400 & -1.90478500 \\ \mathrm{C} & 1.09093100 & -0.41676900 & -2.59202800 \\ \mathrm{C} & 0.40587700 & -0.04388200 & -3.76584800 \\ \mathrm{C} & 0.94898800 & 0.26112900 & -1.36466300 \\ \mathrm{C} & -0.45645300 & 1.05595000 & -3.67586800 \\ \mathrm{C} & 0.07205900 & 1.35253200 & -1.33796800 \\ \mathrm{C} & -0.62366900 & 1.74589000 & -2.47814400 \\ \mathrm{H} & -0.99917600 & 1.37962500 & -4.55860900 \\ \mathrm{H} & -0.06268200 & 1.90545500 & -0.41314400 \\ \mathrm{H} & -1.29448400 & 2.59926600 & -2.43484100 \\ \mathrm{C} & 2.97958800 & -4.96846300 & -2.44770700 \\ \mathrm{C} & 2.78556700 & -5.77334800 & -3.58862100 \\ \mathrm{C} & 3.30645800 & -5.49094800 & -1.18030800 \\ \mathrm{C} & 2.91451600 & -7.15782600 & -3.42030700 \\ \mathrm{C} & 3.42450800 & -6.88260100 & -1.07593900 \\ \mathrm{C} & 3.22789100 & -7.70799000 & -2.18003600 \\ \mathrm{H} & 2.77270900 & -7.81312700 & -4.27453100 \\ & & & \\ & & & \end{array}$

$\begin{array}{lll}3.67766300 & -7.32464700 & -0.11693000\end{array}$

$\begin{array}{llll}3.32659100 & -8.78468600 & -2.07498400\end{array}$

$\begin{array}{llll}1.71507800 & -0.12982400 & -0.10409300\end{array}$

$\begin{array}{lll}2.28865600 & -1.03774700 & -0.31777500\end{array}$

$\begin{array}{lll}2.72444800 & 0.96516100 & 0.29700600\end{array}$

$\begin{array}{llll}3.29779200 & 0.65160300 & 1.17659800\end{array}$

$\begin{array}{lll}3.43237600 & 1.17488700 & -0.51255100\end{array}$

$\begin{array}{llll}2.21783000 & 1.90417200 & 0.54620400\end{array}$

$\begin{array}{lll}0.76631900 & -0.46188100 & 1.06363300\end{array}$

$\begin{array}{lll}0.18388500 & 0.41249800 & 1.37420900\end{array}$

$\begin{array}{lll}0.06159800 & -1.25489200 & 0.78800100\end{array}$

$\begin{array}{lll}1.34058500 & -0.80116500 & 1.93304300\end{array}$

$\begin{array}{llll}3.53993200 & -4.61607700 & 0.04799700\end{array}$

$\begin{array}{lll}4.98888900 & -4.74210900 & 0.55975200\end{array}$

$\begin{array}{llll}5.71363500 & -4.48216900 & -0.21965100\end{array}$

$\begin{array}{lll}5.15053200 & -4.07194700 & 1.41132900\end{array}$

$\begin{array}{llll}5.20990300 & -5.76224900 & 0.89249700\end{array}$

$\begin{array}{llll}2.46362500 & -5.20393600 & -4.96801200\end{array}$

$\begin{array}{llll}2.41608100 & -4.11288000 & -4.88969300\end{array}$

$\begin{array}{lll}0.59772500 & -0.75302900 & -5.10349800\end{array}$

$\begin{array}{llll}1.34361900 & 0.15533500 & -6.10303000\end{array}$

$\begin{array}{llll}0.76178000 & 1.05426300 & -6.33575100\end{array}$

$\begin{array}{llll}2.31243100 & 0.48059600 & -5.70728500\end{array}$

$\begin{array}{lll}1.52290900 & -0.37906300 & -7.04270600\end{array}$

$\begin{array}{lll}1.08775200 & -5.68083400 & -5.47425700\end{array}$

$\begin{array}{lll}0.28324700 & -5.37149500 & -4.79717900\end{array}$

$\begin{array}{llll}0.88355900 & -5.25256100 & -6.46242600\end{array}$

$\begin{array}{llll}1.05365500 & -6.77196300 & -5.57190800\end{array}$

$\begin{array}{llll}3.57448100 & -5.53839700 & -5.98406500\end{array}$

$\begin{array}{lll}3.35689200 & -5.07115000 & -6.95093500\end{array}$

$\begin{array}{llll}4.55137000 & -5.17731000 & -5.64416300\end{array}$

$3.65836600 \quad-6.61829400 \quad-6.14842800$

$\begin{array}{llll}2.52706600 & -4.92972900 & 1.16659200\end{array}$

$\begin{array}{lll}1.49670000 & -4.80645200 & 0.81266600\end{array}$

$\begin{array}{lll}2.63516300 & -5.95619700 & 1.53418300\end{array}$

$\begin{array}{llll}2.67981300 & -4.25543300 & 2.01681000\end{array}$

$\begin{array}{lll}3.38719400 & -3.57072000 & -0.23982700\end{array}$

$-0.73495600 \quad-1.25121300 \quad-5.69471900$ $\begin{array}{lll}-0.54873500 & -1.77838600 & -6.63752700\end{array}$

$\begin{array}{lll}-1.24507500 & -1.94142000 & -5.01472600\end{array}$

$\begin{array}{lll}-1.41632900 & -0.42142900 & -5.91368600\end{array}$

$\begin{array}{lll}1.22339300 & -1.63638600 & -4.93779500\end{array}$

$\begin{array}{lll}-2.36464700 & -4.36534900 & -4.12304500\end{array}$

$\begin{array}{lll}-3.98785700 & -5.52611700 & -3.01521100\end{array}$

$\begin{array}{lll}-3.01686600 & -5.22410500 & -5.07343400\end{array}$

$\begin{array}{lll}-3.77672100 & -4.66399200 & -5.63216200\end{array}$

$-2.26064100 \quad-5.60462600 \quad-5.76340900$

$\begin{array}{lll}-3.63971500 & -6.28782200 & -4.17760200\end{array}$

$\begin{array}{lll}-2.92343900 & -7.08251700 & -3.92737200\end{array}$

$\begin{array}{lll}-4.55601900 & -6.73317800 & -4.56953600\end{array}$

$\begin{array}{lll}-3.00841600 & -4.51948000 & -2.86375400\end{array}$

$-2.00469000 \quad-4.90091600 \quad-1.74886000$

$\begin{array}{lll}-1.59026300 & -5.90257400 & -1.68487800\end{array}$

$\begin{array}{lll}-1.75121600 & -4.17968300 & 0.31140900\end{array}$

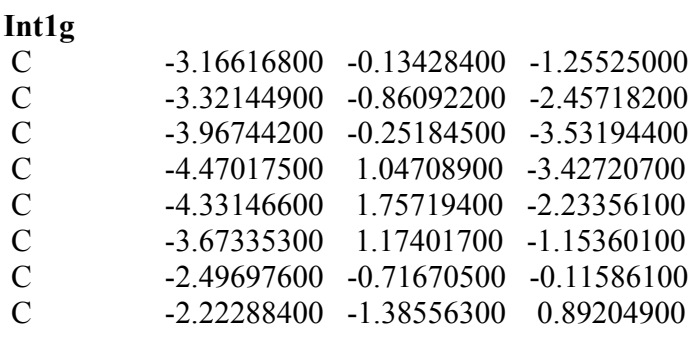




\begin{tabular}{|c|c|c|c|}
\hline $\mathrm{H}$ & -4.07230900 & -0.81211300 & -4.45442000 \\
\hline $\mathrm{H}$ & -4.97214900 & 1.50103600 & -4.27659500 \\
\hline $\mathrm{H}$ & -4.73044000 & 2.76297700 & -2.14271400 \\
\hline $\mathrm{H}$ & -3.54982300 & 1.72035400 & -0.22348500 \\
\hline $\mathrm{C}$ & 1.48574300 & 0.84970900 & 0.17377600 \\
\hline $\mathrm{C}$ & 3.10372200 & 2.39311000 & 0.47188100 \\
\hline $\mathrm{C}$ & 3.65773500 & 1.33920800 & -0.18173700 \\
\hline $\mathrm{H}$ & 3.52568800 & 3.32782100 & 0.80552700 \\
\hline $\mathrm{H}$ & 4.66190900 & 1.16597300 & -0.53466000 \\
\hline $\mathrm{N}$ & 2.65201700 & 0.39953900 & -0.35794300 \\
\hline $\mathrm{N}$ & 1.76969700 & 2.07833300 & 0.68244500 \\
\hline $\mathrm{Au}$ & -0.31959500 & -0.06471600 & 0.21232100 \\
\hline $\mathrm{C}$ & 0.82407300 & 2.95639900 & 1.34362400 \\
\hline $\mathrm{C}$ & 0.15306800 & 3.92226800 & 0.56648300 \\
\hline $\mathrm{C}$ & 0.64491700 & 2.82413000 & 2.73501700 \\
\hline $\mathrm{C}$ & -0.74902900 & 4.76097000 & 1.23297800 \\
\hline $\mathrm{C}$ & -0.27215300 & 3.68980300 & 3.34436700 \\
\hline $\mathrm{C}$ & -0.96475200 & 4.64413100 & 2.60384200 \\
\hline $\mathrm{H}$ & -1.28365700 & 5.52100800 & 0.67131700 \\
\hline $\mathrm{H}$ & -0.43840800 & 3.62078400 & 4.41518600 \\
\hline $\mathrm{H}$ & -1.66748800 & 5.30804700 & 3.09941500 \\
\hline $\mathrm{C}$ & 2.86189700 & -0.87416700 & -1.01849800 \\
\hline $\mathrm{C}$ & 2.68811800 & -0.94077200 & -2.41564100 \\
\hline $\mathrm{C}$ & 3.28975100 & -1.96734200 & -0.23887300 \\
\hline $\mathrm{C}$ & 2.96430700 & -2.16825200 & -3.03221700 \\
\hline $\mathrm{C}$ & 3.54201600 & -3.16987500 & -0.91103700 \\
\hline $\mathrm{C}$ & 3.38641600 & -3.26986200 & -2.29121100 \\
\hline $\mathrm{H}$ & 2.85267400 & -2.25945700 & -4.10812500 \\
\hline $\mathrm{H}$ & 3.87690100 & -4.03549300 & -0.34715400 \\
\hline $\mathrm{H}$ & 3.60094800 & -4.20924900 & -2.79324900 \\
\hline $\mathrm{C}$ & 1.42292600 & 1.82243100 & 3.58371800 \\
\hline $\mathrm{H}$ & 2.04565300 & 1.21691200 & 2.91719600 \\
\hline $\mathrm{C}$ & 2.37069700 & 2.54658200 & 4.56188800 \\
\hline $\mathrm{H}$ & 2.96278500 & 1.81815200 & 5.12724100 \\
\hline $\mathrm{H}$ & 3.06347300 & 3.21047500 & 4.03291400 \\
\hline $\mathrm{H}$ & 1.81300500 & 3.15452300 & 5.28289800 \\
\hline $\mathrm{C}$ & 0.48961400 & 0.85322400 & 4.33370500 \\
\hline $\mathrm{H}$ & -0.15698900 & 1.38214600 & 5.04279800 \\
\hline $\mathrm{H}$ & -0.15160300 & 0.29907300 & 3.63970400 \\
\hline $\mathrm{H}$ & 1.07965300 & 0.12699300 & 4.90425500 \\
\hline $\mathrm{C}$ & 3.50320700 & -1.88295000 & 1.26994800 \\
\hline $\mathrm{C}$ & 4.98006200 & -2.12482400 & 1.64168400 \\
\hline $\mathrm{H}$ & 5.64370100 & -1.41776500 & 1.13173900 \\
\hline $\mathrm{H}$ & 5.12393700 & -2.00743200 & 2.72164200 \\
\hline $\mathrm{H}$ & 5.30215900 & -3.13653700 & 1.37111300 \\
\hline $\mathrm{C}$ & 2.25931000 & 0.25743300 & -3.25757200 \\
\hline $\mathrm{H}$ & 1.92050600 & 1.04733100 & -2.57877500 \\
\hline $\mathrm{C}$ & 0.40338600 & 4.10744800 & -0.92782000 \\
\hline $\mathrm{C}$ & 1.14441000 & 5.43357200 & -1.19792500 \\
\hline $\mathrm{H}$ & 0.53980900 & 6.29577800 & -0.89468400 \\
\hline $\mathrm{H}$ & 2.09258500 & 5.48508900 & -0.65100700 \\
\hline $\mathrm{H}$ & 1.36262900 & 5.53698100 & -2.26672500 \\
\hline $\mathrm{C}$ & 1.07622300 & -0.07374100 & -4.18755900 \\
\hline $\mathrm{H}$ & 0.23328600 & -0.50353100 & -3.63712800 \\
\hline $\mathrm{H}$ & 0.73406300 & 0.83866800 & -4.68916100 \\
\hline $\mathrm{H}$ & 1.36418300 & -0.78346900 & -4.97152500 \\
\hline $\mathrm{C}$ & 3.45247300 & 0.81747700 & -4.06003100 \\
\hline $\mathrm{H}$ & 3.14462000 & 1.69798300 & -4.63508300 \\
\hline $\mathrm{H}$ & 4.28061400 & 1.11274100 & -3.40559700 \\
\hline $\mathrm{H}$ & 3.83645400 & 0.07327500 & -4.76707900 \\
\hline $\mathrm{C}$ & 2.57423500 & -2.84933200 & 2.02981600 \\
\hline $\mathrm{H}$ & 1.52075900 & -2.64417100 & 1.80987400 \\
\hline $\mathrm{H}$ & 2.77800100 & -3.89356900 & 1.76667300 \\
\hline $\mathrm{H}$ & 2.72263000 & -2.74452900 & 3.11069200 \\
\hline $\mathrm{H}$ & 3.24825000 & -0.87014000 & 1.59808000 \\
\hline $\mathrm{C}$ & -0.89343500 & 4.02374200 & -1.75393600 \\
\hline $\mathrm{H}$ & -0.66363700 & 4.11505500 & -2.82149300 \\
\hline
\end{tabular}

$\begin{array}{lrrr}\mathrm{H} & -1.40745200 & 3.06954900 & -1.59836100 \\ \mathrm{H} & -1.59054500 & 4.82988600 & -1.49904000 \\ \mathrm{H} & 1.05254900 & 3.29548100 & -1.27133200 \\ \mathrm{C} & -2.09601000 & -2.17147800 & 2.07576800 \\ \mathrm{C} & -1.54474000 & -3.46584900 & 2.02901400 \\ \mathrm{C} & -2.56778700 & -1.67618700 & 3.31441100 \\ \mathrm{C} & -1.47063600 & -4.25308400 & 3.17265500 \\ \mathrm{H} & -1.17846400 & -3.85618800 & 1.08432100 \\ \mathrm{C} & -2.49307200 & -2.45371700 & 4.45449300 \\ \mathrm{H} & -2.99954500 & -0.68138900 & 3.36225900 \\ \mathrm{C} & -1.94612900 & -3.75130700 & 4.39680300 \\ \mathrm{H} & -1.04895500 & -5.24873900 & 3.10520800 \\ \mathrm{H} & -2.85780000 & -2.08985100 & 5.40924600 \\ \mathrm{O} & -1.92493500 & -4.42622800 & 5.56338500 \\ \mathrm{C} & -1.41006500 & -5.75671700 & 5.58917000 \\ \mathrm{H} & -0.35404200 & -5.77905300 & 5.29456300 \\ \mathrm{H} & -1.50653700 & -6.08864600 & 6.62305500 \\ \mathrm{H} & -1.99119100 & -6.41866600 & 4.93639000 \\ \mathrm{C} & -2.79969100 & -2.28703400 & -2.57521000 \\ \mathrm{H} & -3.12104800 & -2.87637200 & -1.69430800 \\ \mathrm{C} & -0.77012000 & -3.49921900 & -2.56584600 \\ \mathrm{H} & 0.30394100 & -3.31334400 & -2.52438400 \\ \mathrm{H} & -1.07995300 & -4.05877300 & -1.66907100 \\ \mathrm{H} & -1.01397000 & -4.08841300 & -3.45514400 \\ \mathrm{C} & -4.48987300 & -3.62736600 & -3.58992600 \\ \mathrm{H} & -4.43558400 & -4.36812900 & -2.77892100 \\ \mathrm{H} & -5.32483500 & -2.94232000 & -3.38908100 \\ \mathrm{H} & -4.67195700 & -4.14346500 & -4.53459500 \\ \mathrm{O} & -1.40080800 & -2.22108200 & -2.60605500 \\ \mathrm{O} & -3.25701800 & -2.93374800 & -3.73905800\end{array}$

\begin{tabular}{cccc} 
TS1g & & & \\
$\mathrm{C}$ & -3.28273600 & 0.10462400 & -0.13723000 \\
$\mathrm{C}$ & -4.61883300 & -0.26411700 & -0.41161900 \\
$\mathrm{C}$ & -5.63794100 & 0.68970900 & -0.40765200 \\
$\mathrm{C}$ & -5.35700500 & 2.01436500 & -0.07504400 \\
$\mathrm{C}$ & -4.04515400 & 2.38722300 & 0.22419700 \\
$\mathrm{C}$ & -3.01902700 & 1.44485500 & 0.18460200 \\
$\mathrm{C}$ & -2.20818600 & -0.89476400 & -0.28517400 \\
$\mathrm{C}$ & -2.44519500 & -2.09941700 & -0.75687500 \\
$\mathrm{H}$ & -6.65161100 & 0.38468200 & -0.64806700 \\
$\mathrm{H}$ & -6.15731400 & 2.74776700 & -0.05005100 \\
$\mathrm{H}$ & -3.81503200 & 3.41871800 & 0.47430900 \\
$\mathrm{H}$ & -1.99720800 & 1.74911700 & 0.38692500 \\
$\mathrm{C}$ & 1.73367900 & 0.10722400 & 0.55387400 \\
$\mathrm{C}$ & 3.56612700 & 1.39374400 & 0.88708200 \\
$\mathrm{C}$ & 3.93704700 & 0.10081200 & 1.06886100 \\
$\mathrm{H}$ & 4.12748800 & 2.31261400 & 0.94901300 \\
$\mathrm{H}$ & 4.88799800 & -0.33985000 & 1.32326800 \\
$\mathrm{~N}$ & 2.80269600 & -0.67526900 & 0.86205800 \\
$\mathrm{~N}$ & 2.21324800 & 1.38088100 & 0.57356200 \\
$\mathrm{Au}$ & -0.20021400 & -0.44216600 & 0.12577800 \\
$\mathrm{C}$ & 1.42689600 & 2.57087600 & 0.32121500 \\
$\mathrm{C}$ & 1.32096400 & 3.04025600 & -1.00317900 \\
$\mathrm{C}$ & 0.81888300 & 3.21710200 & 1.41686100 \\
$\mathrm{C}$ & 0.55687900 & 4.19492200 & -1.21421200 \\
$\mathrm{C}$ & 0.06899200 & 4.36858000 & 1.14409800 \\
$\mathrm{C}$ & -0.06379300 & 4.85224000 & -0.15536500 \\
$\mathrm{H}$ & 0.45191600 & 4.58569000 & -2.22179600 \\
$\mathrm{H}$ & -0.41066700 & 4.89726300 & 1.96254900 \\
$\mathrm{H}$ & -0.64644300 & 5.75004800 & -0.34228100 \\
$\mathrm{C}$ & 2.78964300 & -2.11783500 & 0.98727000 \\
$\mathrm{C}$ & 3.09600400 & -2.89510000 & -0.14734900 \\
$\mathrm{C}$ & 2.51680100 & -2.67798100 & 2.25159400 \\
$\mathrm{C}$ & 3.11989100 & -4.28573600 & 0.01675600 \\
& & & \\
\hline
\end{tabular}




\begin{tabular}{|c|c|c|c|}
\hline $\mathrm{C}$ & 2.55331700 & -4.07451800 & 2.35433100 \\
\hline $\mathrm{C}$ & 2.85405700 & -4.87079300 & 1.25203600 \\
\hline $\mathrm{H}$ & 3.35618900 & -4.91774600 & -0.83409600 \\
\hline $\mathrm{H}$ & 2.35502400 & -4.54265700 & 3.31409400 \\
\hline $\mathrm{H}$ & 2.88958900 & -5.95171200 & 1.35792400 \\
\hline $\mathrm{C}$ & 0.95971100 & 2.72727700 & 2.85550200 \\
\hline $\mathrm{H}$ & 1.55388700 & 1.80795800 & 2.84836700 \\
\hline $\mathrm{C}$ & 1.71623800 & 3.75314400 & 3.72341800 \\
\hline $\mathrm{H}$ & 1.85303200 & 3.36447400 & 4.73882000 \\
\hline $\mathrm{H}$ & 2.70560100 & 3.97747600 & 3.30940800 \\
\hline $\mathrm{H}$ & 1.16619300 & 4.69779700 & 3.80019300 \\
\hline $\mathrm{C}$ & -0.40605400 & 2.37580000 & 3.47692400 \\
\hline $\mathrm{H}$ & -1.05432000 & 3.25609700 & 3.55360900 \\
\hline $\mathrm{H}$ & -0.93132400 & 1.61964500 & 2.88300000 \\
\hline $\mathrm{H}$ & -0.26899800 & 1.97920000 & 4.48936900 \\
\hline $\mathrm{C}$ & 2.20146400 & -1.83581200 & 3.48460000 \\
\hline $\mathrm{C}$ & 3.24522500 & -2.05149200 & 4.59853200 \\
\hline $\mathrm{H}$ & 4.25925900 & -1.83228100 & 4.24622500 \\
\hline $\mathrm{H}$ & 3.03238700 & -1.39523700 & 5.44988100 \\
\hline $\mathrm{H}$ & 3.23632800 & -3.08379600 & 4.96568200 \\
\hline $\mathrm{C}$ & 3.40696300 & -2.28630300 & -1.51152800 \\
\hline $\mathrm{H}$ & 3.32445500 & -1.19816300 & -1.42739500 \\
\hline $\mathrm{C}$ & 1.99993400 & 2.35501400 & -2.18524900 \\
\hline $\mathrm{C}$ & 3.01988000 & 3.28978800 & -2.86592600 \\
\hline $\mathrm{H}$ & 2.53190900 & 4.16826400 & -3.30282900 \\
\hline $\mathrm{H}$ & 3.77381200 & 3.64691300 & -2.15553600 \\
\hline $\mathrm{H}$ & 3.53807300 & 2.76194600 & -3.67448100 \\
\hline $\mathrm{C}$ & 2.38766700 & -2.73564300 & -2.57659400 \\
\hline $\mathrm{H}$ & 1.36577500 & -2.47024100 & -2.28439400 \\
\hline $\mathrm{H}$ & 2.60632200 & -2.25312900 & -3.53611700 \\
\hline $\mathrm{H}$ & 2.42270100 & -3.81970300 & -2.73458100 \\
\hline $\mathrm{C}$ & 4.84969600 & -2.60328100 & -1.95301400 \\
\hline $\mathrm{H}$ & 5.06998900 & -2.11558200 & -2.90926500 \\
\hline $\mathrm{H}$ & 5.58135100 & -2.25274600 & -1.21653700 \\
\hline $\mathrm{H}$ & 5.00226600 & -3.68026900 & -2.08616500 \\
\hline $\mathrm{C}$ & 0.77402200 & -2.10449300 & 4.00092300 \\
\hline $\mathrm{H}$ & 0.02759200 & -1.89168400 & 3.22699300 \\
\hline $\mathrm{H}$ & 0.64995500 & -3.14646700 & 4.31790500 \\
\hline $\mathrm{H}$ & 0.55682800 & -1.46734500 & 4.86581000 \\
\hline $\mathrm{H}$ & 2.24517100 & -0.77992900 & 3.19997800 \\
\hline $\mathrm{C}$ & 0.96671700 & 1.82564700 & -3.19940800 \\
\hline $\mathrm{H}$ & 1.47313900 & 1.30022400 & -4.01725500 \\
\hline $\mathrm{H}$ & 0.26801300 & 1.12777200 & -2.72420800 \\
\hline $\mathrm{H}$ & 0.38239400 & 2.64137600 & -3.64018100 \\
\hline $\mathrm{H}$ & 2.55473500 & 1.49003300 & -1.80805100 \\
\hline $\mathrm{C}$ & -1.92954900 & -3.43171100 & -0.93485600 \\
\hline $\mathrm{C}$ & -2.09649100 & -4.11078400 & -2.15833600 \\
\hline $\mathrm{C}$ & -1.25283300 & -4.09869000 & 0.11555300 \\
\hline $\mathrm{C}$ & -1.60988700 & -5.39971500 & -2.34376500 \\
\hline $\mathrm{H}$ & -2.61305100 & -3.61333100 & -2.97473500 \\
\hline $\mathrm{C}$ & -0.75068900 & -5.37347400 & -0.06560800 \\
\hline $\mathrm{H}$ & -1.11528600 & -3.58944400 & 1.06376900 \\
\hline $\mathrm{C}$ & -0.92670300 & -6.04044900 & -1.29445300 \\
\hline $\mathrm{H}$ & -1.75206600 & -5.89192900 & -3.29845300 \\
\hline $\mathrm{H}$ & -0.21346200 & -5.88316300 & 0.72670800 \\
\hline $\mathrm{O}$ & -0.40584200 & -7.28274000 & -1.36327700 \\
\hline $\mathrm{C}$ & -0.55002600 & -8.03238700 & -2.56917800 \\
\hline $\mathrm{H}$ & -0.05971400 & -7.52859700 & -3.41060600 \\
\hline $\mathrm{H}$ & -0.06048500 & -8.98821500 & -2.38133800 \\
\hline $\mathrm{H}$ & -1.60691900 & -8.20312000 & -2.80555400 \\
\hline $\mathrm{O}$ & -5.72838700 & -1.80006500 & -1.82812100 \\
\hline $\mathrm{O}$ & -5.30880300 & -2.37578500 & 0.41093100 \\
\hline $\mathrm{C}$ & -6.65382800 & -2.88325100 & -1.98806400 \\
\hline $\mathrm{H}$ & -7.22945300 & -2.63409100 & -2.87977800 \\
\hline $\mathrm{H}$ & -6.13472100 & -3.83410800 & -2.14789200 \\
\hline $\mathrm{C}$ & -5.16666000 & -3.80754200 & 0.49905100 \\
\hline $\mathrm{H}$ & -4.52792400 & -4.19758600 & -0.29645700 \\
\hline
\end{tabular}

$\begin{array}{llll}\mathrm{H} & -4.70706200 & -4.01627500 & 1.46699500 \\ \mathrm{C} & -4.95195700 & -1.69040600 & -0.71244100 \\ \mathrm{H} & -3.77851200 & -2.12852000 & -1.06534300 \\ \mathrm{H} & -7.32108700 & -2.95612400 & -1.12533500 \\ \mathrm{H} & -6.15090200 & -4.28052800 & 0.4534300\end{array}$

Imaginary Frequency $=-710.4896$

\begin{tabular}{|c|c|c|c|}
\hline nt2g & & & \\
\hline $\mathrm{C}$ & -4.58069000 & -0.17551400 & -2.93534400 \\
\hline $\mathrm{C}$ & -5.43890300 & -1.26619000 & -3.22367800 \\
\hline $\mathrm{C}$ & -6.67462600 & -1.06131000 & -3.81749800 \\
\hline $\mathrm{C}$ & -7.07337800 & 0.25917900 & -4.06580000 \\
\hline $\mathrm{C}$ & -6.25132300 & 1.35688500 & -3.74603900 \\
\hline $\mathrm{C}$ & -5.00004500 & 1.15330500 & -3.18373100 \\
\hline $\mathrm{C}$ & -3.29767400 & -0.62349500 & -2.51112700 \\
\hline $\mathrm{C}$ & -3.25882700 & -2.12583600 & -2.65427000 \\
\hline $\mathrm{H}$ & -7.31293300 & -1.89613900 & -4.08603800 \\
\hline $\mathrm{H}$ & -8.04512000 & 0.44282900 & -4.51637100 \\
\hline $\mathrm{H}$ & -6.59885000 & 2.36329200 & -3.95703400 \\
\hline $\mathrm{H}$ & -4.33868300 & 1.98533400 & -2.96048700 \\
\hline $\mathrm{C}$ & -0.26820800 & 1.89740300 & -1.41100900 \\
\hline $\mathrm{C}$ & 0.90205900 & 3.82865400 & -1.25319300 \\
\hline $\mathrm{C}$ & 1.61919600 & 2.86140300 & -0.62347900 \\
\hline $\mathrm{H}$ & 1.09968800 & 4.87796900 & -1.40585500 \\
\hline $\mathrm{H}$ & 2.56912600 & 2.89596300 & -0.11378600 \\
\hline $\mathrm{N}$ & 0.88890200 & 1.68631100 & -0.72877700 \\
\hline $\mathrm{N}$ & -0.25070400 & 3.22204300 & -1.72862400 \\
\hline $\mathrm{Au}$ & -1.77638100 & 0.57333300 & -1.92979000 \\
\hline $\mathrm{C}$ & -1.30056800 & 3.91588500 & -2.44621600 \\
\hline $\mathrm{C}$ & -1.21509100 & 4.00207900 & -3.85026800 \\
\hline $\mathrm{C}$ & -2.35421800 & 4.48673000 & -1.70332500 \\
\hline $\mathrm{C}$ & -2.25004300 & 4.67325600 & -4.51428900 \\
\hline $\mathrm{C}$ & -3.36068200 & 5.14501100 & -2.42222900 \\
\hline $\mathrm{C}$ & -3.31270300 & 5.23572700 & -3.81159000 \\
\hline $\mathrm{H}$ & -2.21856500 & 4.76161600 & -5.59615900 \\
\hline $\mathrm{H}$ & -4.18570300 & 5.60254400 & -1.88418300 \\
\hline $\mathrm{H}$ & -4.09998500 & 5.75840700 & -4.34791300 \\
\hline $\mathrm{C}$ & 1.31891700 & 0.42743900 & -0.15246600 \\
\hline $\mathrm{C}$ & 2.10277400 & -0.43958100 & -0.93853600 \\
\hline $\mathrm{C}$ & 0.97202300 & 0.15108700 & 1.18546300 \\
\hline $\mathrm{C}$ & 2.53914100 & -1.62708000 & -0.33802200 \\
\hline $\mathrm{C}$ & 1.43556900 & -1.05241100 & 1.73009500 \\
\hline $\mathrm{C}$ & 2.21036000 & -1.93237000 & 0.97970100 \\
\hline $\mathrm{H}$ & 3.14916100 & -2.31824400 & -0.91203700 \\
\hline $\mathrm{H}$ & 1.18721700 & -1.30304100 & 2.75699200 \\
\hline $\mathrm{H}$ & 2.55727100 & -2.85950400 & 1.42630200 \\
\hline $\mathrm{C}$ & -2.42577200 & 4.42936500 & -0.17968700 \\
\hline $\mathrm{H}$ & -1.55359500 & 3.87777000 & 0.18492800 \\
\hline $\mathrm{C}$ & -2.36161000 & 5.84123700 & 0.43722500 \\
\hline $\mathrm{H}$ & -2.35610500 & 5.77645900 & 1.53098100 \\
\hline $\mathrm{H}$ & -1.45776900 & 6.37517800 & 0.12371000 \\
\hline $\mathrm{H}$ & -3.22520200 & 6.44904700 & 0.14493900 \\
\hline $\mathrm{C}$ & -3.67580000 & 3.66851000 & 0.30340500 \\
\hline $\mathrm{H}$ & -4.59902400 & 4.16800800 & -0.01190800 \\
\hline $\mathrm{H}$ & -3.69216600 & 2.64464600 & -0.08756200 \\
\hline $\mathrm{H}$ & -3.68680400 & 3.61389000 & 1.39778900 \\
\hline $\mathrm{C}$ & 0.13544200 & 1.09663600 & 2.04260900 \\
\hline $\mathrm{C}$ & 0.93729000 & 1.62060000 & 3.25069800 \\
\hline $\mathrm{H}$ & 1.85525000 & 2.12847500 & 2.93442000 \\
\hline $\mathrm{H}$ & 0.33573900 & 2.33300000 & 3.82647600 \\
\hline $\mathrm{H}$ & 1.22391800 & 0.80631300 & 3.92556300 \\
\hline $\mathrm{C}$ & 2.48763600 & -0.13503000 & -2.38319900 \\
\hline $\mathrm{H}$ & 2.04295400 & 0.82539900 & -2.66285200 \\
\hline $\mathrm{C}$ & -0.05621600 & 3.41450600 & -4.65072600 \\
\hline $\mathrm{C}$ & 0.72793500 & 4.51685300 & -5.39087500 \\
\hline
\end{tabular}




$\begin{array}{lrrr}\mathrm{H} & 0.10375500 & 5.02405400 & -6.13489700 \\ \mathrm{H} & 1.10321000 & 5.27750700 & -4.69730500 \\ \mathrm{H} & 1.58613500 & 4.08373400 & -5.91668100 \\ \mathrm{C} & 1.92040200 & -1.19312500 & -3.34969100 \\ \mathrm{H} & 0.83297400 & -1.27909900 & -3.24647400 \\ \mathrm{H} & 2.15005400 & -0.92184000 & -4.38658000 \\ \mathrm{H} & 2.35349300 & -2.18205200 & -3.16157800 \\ \mathrm{C} & 4.01460900 & 0.00394500 & -2.54425000 \\ \mathrm{H} & 4.26582100 & 0.26611700 & -3.57818900 \\ \mathrm{H} & 4.41825500 & 0.78344400 & -1.88846800 \\ \mathrm{H} & 4.53074700 & -0.93234400 & -2.30404000 \\ \mathrm{C} & -1.17987500 & 0.43356800 & 2.49607600 \\ \mathrm{H} & -1.76275800 & 0.08190500 & 1.63783200 \\ \mathrm{H} & -0.99252600 & -0.42604800 & 3.14941700 \\ \mathrm{H} & -1.79082500 & 1.14930100 & 3.05814300 \\ \mathrm{H} & -0.13637600 & 1.96474400 & 1.43327600 \\ \mathrm{C} & -0.53523100 & 2.32289900 & -5.62751800 \\ \mathrm{H} & 0.31971500 & 1.88884800 & -6.15808200 \\ \mathrm{H} & -1.05298000 & 1.51588400 & -5.09658400 \\ \mathrm{H} & -1.22110900 & 2.72773100 & -6.38032800 \\ \mathrm{H} & 0.63715300 & 2.93758200 & -3.95091400 \\ \mathrm{C} & -2.37217100 & -2.87131800 & -1.68433200 \\ \mathrm{C} & -1.37134100 & -3.72793100 & -2.15276500 \\ \mathrm{C} & -2.50715400 & -2.71469300 & -0.29206400 \\ \mathrm{C} & -0.53283600 & -4.42646800 & -1.28147100 \\ \mathrm{H} & -1.23459100 & -3.86217800 & -3.22389200 \\ \mathrm{C} & -1.67928600 & -3.39652200 & 0.58725900 \\ \mathrm{H} & -3.28532900 & -2.06959500 & 0.10235500 \\ \mathrm{C} & -0.68490800 & -4.26141400 & 0.10092200 \\ \mathrm{H} & 0.22597000 & -5.08518900 & -1.68736500 \\ \mathrm{H} & -1.78716300 & -3.28509700 & 1.66129500 \\ \mathrm{O} & 0.06973000 & -4.88546400 & 1.04362100 \\ \mathrm{C} & 1.02045600 & -5.85454800 & 0.62013400 \\ \mathrm{H} & 1.80719200 & -5.40358000 & 0.00134300 \\ \mathrm{H} & 1.46384800 & -6.25615800 & 1.53249100 \\ \mathrm{H} & 0.54116700 & -6.66757100 & 0.06063900 \\ \mathrm{C} & -4.78550100 & -2.56345500 & -2.78531500 \\ \mathrm{H} & -2.84842800 & -2.26739900 & -3.66955800 \\ \mathrm{H} & -4.52715700 & -4.83679500 & -3.54962200 \\ \mathrm{C} & -5.07813900 & -5.49865400 & -4.22035400 \\ \mathrm{H} & -3.46363900 & -4.86280700 & -3.81152200 \\ \mathrm{H} & -4.65036100 & -5.16740200 & -2.51442100 \\ \mathrm{H} & -6.56707500 & -3.24264300 & -1.29697000 \\ \mathrm{H} & -6.64211700 & -3.66629800 & -0.29399100 \\ \mathrm{H} & -6.18659900 & -2.33912600 & -1.35419600 \\ \mathrm{H} & & -3.97393400 & -2.03024100 \\ \mathrm{H} & -3257500 & -3.52917900 & -3.75069100 \\ \mathrm{H} & -2.94093300 & -1.48540900\end{array}$

$\begin{array}{lrrr}\text { TS2g } & & & \\ \text { C } & 0.00139300 & -3.72772100 & -0.21558400 \\ \text { C } & 1.06215100 & -4.63585400 & 0.01049500 \\ \text { C } & 0.83877100 & -6.00808000 & 0.10935100 \\ \text { C } & -0.47912300 & -6.46567200 & 0.06661200 \\ \text { C } & -1.55066300 & -5.56919200 & -0.09688300 \\ \text { C } & -1.32519000 & -4.20416200 & -0.22707500 \\ \text { C } & 0.46403400 & -2.37910300 & -0.46431200 \\ \text { C } & 1.81171100 & -2.43249300 & -0.96072700 \\ \text { H } & 1.66582100 & -6.69672200 & 0.24918900 \\ \text { H } & -0.68212500 & -7.52609100 & 0.18457000 \\ \text { H } & -2.56599500 & -5.95318100 & -0.12839400 \\ \text { H } & -2.14863600 & -3.51310200 & -0.38055200 \\ \text { C } & -1.99407300 & 0.87237600 & 0.07220500 \\ \text { C } & -3.90691300 & 2.08473500 & 0.04546400\end{array}$

$\begin{array}{lll}-2.90508700 & 2.91102800 & 0.44162400\end{array}$ $\begin{array}{lll}-4.96136900 & 2.25941100 & -0.09937300\end{array}$ $\begin{array}{lll}-2.90637600 & 3.95441300 & 0.71481500\end{array}$ $\begin{array}{lll}-1.74057400 & 2.15376600 & 0.45206900\end{array}$ $\begin{array}{llll}-3.33291200 & 0.84021000 & -0.17459000\end{array}$ $\begin{array}{lll}-0.69748500 & -0.73082500 & -0.14182300\end{array}$ $\begin{array}{lll}-4.07291400 & -0.32983000 & -0.60128900\end{array}$ $\begin{array}{lll}-4.20270800 & -0.57903600 & -1.98226800\end{array}$ $\begin{array}{lll}-4.64860000 & -1.15543800 & 0.38577900\end{array}$ $\begin{array}{lll}-4.92849800 & -1.71535400 & -2.36206900\end{array}$ $\begin{array}{lll}-5.36207800 & -2.27781900 & -0.05433600\end{array}$ $\begin{array}{llll}-5.50014500 & -2.55760300 & -1.41193300\end{array}$ $\begin{array}{lll}-5.05109100 & -1.93928900 & -3.41752700\end{array}$ $\begin{array}{lll}-5.82310000 & -2.93497400 & 0.67711300\end{array}$ $\begin{array}{lll}-6.06407800 & -3.42976800 & -1.73101200\end{array}$ $\begin{array}{lll}-0.44947200 & 2.68606300 & 0.83821800\end{array}$ $\begin{array}{llll}0.35940200 & 3.27575800 & -0.15322900\end{array}$ $\begin{array}{lll}-0.08303400 & 2.63519500 & 2.19851500\end{array}$ $\begin{array}{lll}1.57937700 & 3.82597400 & 0.26002200\end{array}$ $\begin{array}{lll}1.15020000 & 3.19753600 & 2.55116300\end{array}$ $\begin{array}{lll}1.97193000 & 3.79028500 & 1.59548600\end{array}$ $\begin{array}{llll}2.22898200 & 4.29026500 & -0.47558700\end{array}$ $\begin{array}{lll}1.46467700 & 3.18036200 & 3.59055500\end{array}$ $\begin{array}{lll}2.91910500 & 4.23146200 & 1.89362300\end{array}$ $\begin{array}{lll}-4.53287300 & -0.86997900 & 1.88081200\end{array}$ $\begin{array}{lll}-3.95025400 & 0.04738500 & 2.01073400\end{array}$ $\begin{array}{lll}-5.91669900 & -0.62615600 & 2.51565800\end{array}$ $\begin{array}{lll}-5.80847100 & -0.37174600 & 3.57598900\end{array}$ $\begin{array}{lll}-6.44561900 & 0.19617000 & 2.02134100\end{array}$ $\begin{array}{lll}-6.55179100 & -1.51657000 & 2.45075100\end{array}$ $\begin{array}{lll}-3.77592800 & -1.99414000 & 2.61475300\end{array}$ $\begin{array}{lll}-4.30762800 & -2.94999700 & 2.54449400\end{array}$ $\begin{array}{lll}-2.77229300 & -2.13297800 & 2.19687300\end{array}$ $\begin{array}{lll}-3.67303200 & -1.75021500 & 3.67832400\end{array}$ $\begin{array}{lll}-0.96728200 & 2.01601500 & 3.27742800\end{array}$ $\begin{array}{lll}-1.39380700 & 3.06162200 & 4.32732400\end{array}$ $\begin{array}{lll}-1.91720200 & 3.90478200 & 3.86317900\end{array}$ $\begin{array}{lll}-2.06670700 & 2.60768700 & 5.06356200\end{array}$ $\begin{array}{lll}-0.53085300 & 3.46372400 & 4.86967800\end{array}$ $\begin{array}{lll}-0.04164500 & 3.34260200 & -1.62399000\end{array}$ $\begin{array}{lll}-1.01258400 & 2.84987300 & -1.73770500\end{array}$ $\begin{array}{lll}-3.60137500 & 0.32714800 & -3.05257000\end{array}$ $\begin{array}{llll}-4.69694900 & 0.95712000 & -3.93611100\end{array}$ $\begin{array}{lll}-5.41806800 & 1.52507400 & -3.33782600\end{array}$ $\begin{array}{llll}-4.24828400 & 1.64007000 & -4.66618000\end{array}$ $\begin{array}{lll}-5.25327600 & 0.19483400 & -4.49297800\end{array}$ $\begin{array}{llll}0.95964900 & 2.58297700 & -2.51619000\end{array}$ $\begin{array}{lll}1.05667100 & 1.53742800 & -2.20492100\end{array}$ $\begin{array}{lll}0.62525800 & 2.60322100 & -3.55985200\end{array}$ $\begin{array}{llll}1.95652600 & 3.03632800 & -2.47769200\end{array}$ $\begin{array}{lll}-0.21069400 & 4.80129600 & -2.09436400\end{array}$ $\begin{array}{lll}-0.54380200 & 4.82749200 & -3.13798500\end{array}$ $\begin{array}{lll}-0.95031900 & 5.33660400 & -1.48842800\end{array}$ $\begin{array}{llll}0.73299300 & 5.35470800 & -2.03260500\end{array}$ $\begin{array}{lll}-0.27890300 & 0.80989700 & 3.94592500\end{array}$ $\begin{array}{lll}-0.02366000 & 0.04265400 & 3.20575000\end{array}$ $\begin{array}{llll}0.64295200 & 1.10698200 & 4.45881900\end{array}$ $\begin{array}{lll}-0.94329100 & 0.35798600 & 4.69131200\end{array}$ $\begin{array}{lll}-1.88017900 & 1.64281800 & 2.80290000\end{array}$ $\begin{array}{lll}-2.56184900 & -0.42338800 & -3.90804300\end{array}$ $\begin{array}{lll}-2.10942900 & 0.25779400 & -4.63764700\end{array}$ $\begin{array}{lll}-1.76159000 & -0.83793100 & -3.28449900\end{array}$ $\begin{array}{lll}-3.01969000 & -1.24911400 & -4.46449900\end{array}$ $\begin{array}{lll}-3.07634800 & 1.14786000 & -2.55364500\end{array}$ $\begin{array}{lll}2.77002900 & -1.30992900 & -1.00616200\end{array}$ $\begin{array}{lll}3.71162800 & -1.25465100 & -2.04721800\end{array}$ $\begin{array}{llll}2.83337800 & -0.30857400 & -0.01620900\end{array}$ 


$\begin{array}{crrr}\mathrm{C} & 4.67160300 & -0.24636500 & -2.12211500 \\ \mathrm{H} & 3.69118600 & -2.01605500 & -2.82407500 \\ \mathrm{C} & 3.78098000 & 0.70091200 & -0.07539000 \\ \mathrm{H} & 2.12825900 & -0.31584800 & 0.80904000 \\ \mathrm{C} & 4.70985100 & 0.74417900 & -1.12977400 \\ \mathrm{H} & 5.37315600 & -0.23799800 & -2.94813700 \\ \mathrm{H} & 3.82343700 & 1.47418000 & 0.68390900 \\ \mathrm{O} & 5.59302600 & 1.77267700 & -1.09372300 \\ \mathrm{C} & 6.57727900 & 1.86583200 & -2.11819700 \\ \mathrm{H} & 6.11616400 & 1.99152600 & -3.10579700 \\ \mathrm{H} & 7.17067800 & 2.74953100 & -1.88032700 \\ \mathrm{H} & 7.22810300 & 0.98270700 & -2.12849900 \\ \mathrm{C} & 2.39494000 & -3.96056500 & 0.08825800 \\ \mathrm{H} & 1.88466800 & -3.06829900 & -1.84546200 \\ \mathrm{C} & 1.93415600 & -3.04949900 & 2.26687800 \\ \mathrm{H} & 2.54440300 & -2.70073400 & 3.10005000 \\ \mathrm{H} & 1.30472300 & -2.23031100 & 1.89430400 \\ \mathrm{H} & 1.29149900 & -3.87857900 & 2.58082300 \\ \mathrm{C} & 4.74191900 & -4.42686700 & -0.22124200 \\ \mathrm{H} & 5.30229900 & -5.02405800 & -0.94050700 \\ \mathrm{H} & 5.01932700 & -3.37379600 & -0.30481000 \\ \mathrm{H} & 4.92816500 & -4.78191000 & 0.79478900 \\ \mathrm{O} & 3.35900800 & -4.62112900 & -0.58474600 \\ \mathrm{O} & 2.85823600 & -3.47376700 & 1.26912900 \\ \mathrm{Imaginary} & \text { Frequency }=-288.0370 & \\ & & & \end{array}$

\begin{tabular}{crrr} 
Int3g & & & \\
$\mathrm{C}$ & -0.06851600 & 3.35213600 & 0.33121900 \\
$\mathrm{C}$ & -1.02285200 & 4.38930000 & 0.18081900 \\
$\mathrm{C}$ & -0.66557500 & 5.71636100 & 0.36266000 \\
$\mathrm{C}$ & 0.64951900 & 5.99471900 & 0.75876000 \\
$\mathrm{C}$ & 1.59990400 & 4.97375200 & 0.95229700 \\
$\mathrm{C}$ & 1.25465800 & 3.64798800 & 0.73741300 \\
$\mathrm{C}$ & -0.59736300 & 2.09643800 & -0.08136900 \\
$\mathrm{C}$ & -1.96480000 & 2.33951700 & -0.67374400 \\
$\mathrm{H}$ & -1.37931000 & 6.51669400 & 0.19980200 \\
$\mathrm{H}$ & 0.94571100 & 7.02745900 & 0.92223300 \\
$\mathrm{H}$ & 2.60867700 & 5.23445200 & 1.25648700 \\
$\mathrm{H}$ & 1.98218100 & 2.84934000 & 0.84722400 \\
$\mathrm{C}$ & 1.47532500 & -1.43126800 & 0.08173500 \\
$\mathrm{C}$ & 3.24255400 & -2.84515800 & 0.06592300 \\
$\mathrm{C}$ & 2.11922800 & -3.59470900 & 0.21523800 \\
$\mathrm{H}$ & 4.28332800 & -3.12250600 & 0.00868100 \\
$\mathrm{H}$ & 1.98057200 & -4.65939300 & 0.31796900 \\
$\mathrm{~N}$ & 1.04705200 & -2.71410300 & 0.22170600 \\
$\mathrm{~N}$ & 2.83087900 & -1.52284000 & -0.01128400 \\
$\mathrm{Au}$ & 0.37684200 & 0.32638600 & 0.00893100 \\
$\mathrm{C}$ & 3.73234500 & -0.39815300 & -0.15389600 \\
$\mathrm{C}$ & 4.03633400 & 0.06355500 & -1.45024300 \\
$\mathrm{C}$ & 4.28190000 & 0.17094700 & 1.01338900 \\
$\mathrm{C}$ & 4.91095400 & 1.15296600 & -1.55287500 \\
$\mathrm{C}$ & 5.15127900 & 1.25694500 & 0.84755100 \\
$\mathrm{C}$ & 5.46091200 & 1.74599600 & -0.41951200 \\
$\mathrm{H}$ & 5.16943300 & 1.53659900 & -2.53524400 \\
$\mathrm{H}$ & 5.59944500 & 1.71803000 & 1.72295200 \\
$\mathrm{H}$ & 6.14277700 & 2.58535800 & -0.52446200 \\
$\mathrm{C}$ & -0.33228100 & -3.13152600 & 0.37491800 \\
$\mathrm{C}$ & -1.05178300 & -3.50302000 & -0.77817200 \\
$\mathrm{C}$ & -0.87766000 & -3.18527200 & 1.67316400 \\
$\mathrm{C}$ & -2.37263800 & -3.93003300 & -0.59619600 \\
$\mathrm{C}$ & -2.20534700 & -3.61448700 & 1.79038600 \\
$\mathrm{C}$ & -2.94689100 & -3.98188300 & 0.67094600 \\
$\mathrm{H}$ & -2.95830000 & -4.22781200 & -1.46095200 \\
$\mathrm{H}$ & -2.66317800 & -3.66638000 & 2.77364700 \\
$\mathrm{H}$ & -3.97799000 & -4.30192400 & 0.78613200 \\
& & & \\
\hline
\end{tabular}

$\begin{array}{lll}3.98566000 & -0.35564000 & 2.41512500\end{array}$ $\begin{array}{lll}3.27416200 & -1.18292600 & 2.32880500\end{array}$ $\begin{array}{lll}5.25827400 & -0.91699800 & 3.08120700\end{array}$ $\begin{array}{lll}5.01748200 & -1.34258300 & 4.06177300\end{array}$ $\begin{array}{llll}5.71592300 & -1.70449700 & 2.47224700\end{array}$ $\begin{array}{lll}6.01120400 & -0.13553400 & 3.23361500\end{array}$ $\begin{array}{llll}3.32394200 & 0.71697900 & 3.30171500\end{array}$ $\begin{array}{llll}3.98385800 & 1.57847800 & 3.45495300\end{array}$ $\begin{array}{lll}2.38978700 & 1.07843200 & 2.85674500\end{array}$ $\begin{array}{llll}3.08997800 & 0.30147900 & 4.28832100\end{array}$ $\begin{array}{lll}-0.08830200 & -2.81152900 & 2.92489000\end{array}$ $\begin{array}{llll}0.04421600 & -4.00863700 & 3.88723900\end{array}$ $\begin{array}{lll}0.51576100 & -4.86731600 & 3.39659700\end{array}$ $\begin{array}{lll}0.65686200 & -3.73305000 & 4.75308300\end{array}$ $\begin{array}{llll}-0.93199800 & -4.33492500 & 4.26248300\end{array}$ $\begin{array}{lll}-0.45075800 & -3.46767500 & -2.18036600\end{array}$ $\begin{array}{lll}0.56424900 & -3.06360700 & -2.10906500\end{array}$ $\begin{array}{lll}3.47181500 & -0.57659100 & -2.71533100\end{array}$ $\begin{array}{llll}4.59261000 & -1.18054100 & -3.58524500\end{array}$ $\begin{array}{lll}5.28170800 & -0.40929500 & -3.94703800\end{array}$ $\begin{array}{lll}5.18076100 & -1.91741800 & -3.02720900\end{array}$ $\begin{array}{llll}4.16419200 & -1.68036300 & -4.46116700\end{array}$ $\begin{array}{lll}-1.24133200 & -2.53364900 & -3.11695900\end{array}$ $\begin{array}{lll}-1.30520400 & -1.51994500 & -2.70686200\end{array}$ $\begin{array}{lll}-0.75288800 & -2.48059100 & -4.09681600\end{array}$ $\begin{array}{lll}-2.26389200 & -2.89385300 & -3.27650900\end{array}$ $\begin{array}{lll}-0.33675700 & -4.88504100 & -2.77674600\end{array}$ $\begin{array}{llll}0.14090600 & -4.84654800 & -3.76229900\end{array}$ $\begin{array}{llll}0.25956900 & -5.54424100 & -2.13597600\end{array}$ $\begin{array}{lll}-1.32225500 & -5.34763300 & -2.90223700\end{array}$ $\begin{array}{lll}-0.70312100 & -1.59112100 & 3.63783100\end{array}$ $\begin{array}{lll}-0.75140000 & -0.72485400 & 2.96811700\end{array}$ $\begin{array}{lll}-1.71882900 & -1.80186500 & 3.99123200\end{array}$ $\begin{array}{lll}-0.09834200 & -1.31764400 & 4.51007400\end{array}$ $\begin{array}{lll}0.92482900 & -2.52846600 & 2.62136600\end{array}$ $\begin{array}{llll}2.61850900 & 0.41986900 & -3.52431100\end{array}$ $\begin{array}{lll}2.19342600 & -0.07480000 & -4.40497600\end{array}$ $\begin{array}{lll}1.79257300 & 0.81482800 & -2.92152200\end{array}$ $\begin{array}{lll}3.21546100 & 1.26866500 & -3.87680800\end{array}$ $\begin{array}{lll}2.81290100 & -1.39893000 & -2.41881100\end{array}$ $\begin{array}{lll}-2.97190200 & 1.22157100 & -0.53205800\end{array}$ $\begin{array}{llll}-3.59720400 & 0.68438100 & -1.66133300\end{array}$ $\begin{array}{lll}-3.30187300 & 0.67929400 & 0.72427900\end{array}$ $\begin{array}{lll}-4.53252600 & -0.34839400 & -1.56406100\end{array}$ $\begin{array}{lll}-3.35714300 & 1.07566200 & -2.64788000\end{array}$ $\begin{array}{lll}-4.22412900 & -0.34992100 & 0.83831400\end{array}$ $\begin{array}{lll}-2.84663300 & 1.08575200 & 1.62126400\end{array}$ $\begin{array}{lll}-4.84982600 & -0.87333900 & -0.30511600\end{array}$ $\begin{array}{lll}-4.99854200 & -0.73027000 & -2.46486400\end{array}$ $\begin{array}{lll}-4.48518700 & -0.76502900 & 1.80621100\end{array}$ $\begin{array}{lll}-5.73816900 & -1.87936500 & -0.08615700\end{array}$ $\begin{array}{llll}-6.47706300 & -2.38452200 & -1.19155100\end{array}$ $\begin{array}{lll}-5.81958500 & -2.85407600 & -1.93488400\end{array}$ $\begin{array}{lll}-7.15277600 & -3.13608400 & -0.78079300\end{array}$ $\begin{array}{lll}-7.06368900 & -1.59364900 & -1.67525700\end{array}$ $\begin{array}{llll}-2.37296900 & 3.79864200 & -0.18113200\end{array}$ $\begin{array}{lll}-1.74594000 & 2.46590600 & -1.74887000\end{array}$ $\begin{array}{llll}-4.23349600 & 4.24877800 & -1.64966100\end{array}$ $\begin{array}{lll}-4.64096300 & 5.13560000 & -2.13855200\end{array}$ $\begin{array}{lll}-4.13289700 & 3.44496900 & -2.38725300\end{array}$ $\begin{array}{llll}-4.90483100 & 3.92139200 & -0.85056200\end{array}$ $\begin{array}{llll}-3.56640900 & 4.77008900 & 1.68418700\end{array}$ $\begin{array}{lll}-4.33078300 & 4.44112500 & 2.39035700\end{array}$ $\begin{array}{lll}-2.71841700 & 5.18895200 & 2.24009700\end{array}$ $\begin{array}{lll}-3.98382200 & 5.53782400 & 1.02459000\end{array}$ $\begin{array}{lll}-3.18661400 & 3.60151900 & 0.95351600\end{array}$ $\begin{array}{llll}-2.95504100 & 4.63700200 & -1.13420300\end{array}$ 


\begin{tabular}{|c|c|c|c|}
\hline TS3g & & & \\
\hline $\mathrm{C}$ & -0.39038200 & 3.48666400 & 0.04770500 \\
\hline $\mathrm{C}$ & -1.31593400 & 4.53716400 & -0.00585100 \\
\hline $\mathrm{C}$ & -0.90476400 & 5.85888900 & 0.09200700 \\
\hline $\mathrm{C}$ & 0.45704800 & 6.11773900 & 0.30205200 \\
\hline $\mathrm{C}$ & 1.37995000 & 5.07077100 & 0.38739700 \\
\hline $\mathrm{C}$ & 0.96605900 & 3.74174800 & 0.24414900 \\
\hline $\mathrm{C}$ & -1.05268500 & 2.17989600 & -0.23229100 \\
\hline $\mathrm{C}$ & -2.42772100 & 2.45751600 & -0.47896600 \\
\hline $\mathrm{H}$ & -1.61441200 & 6.67515700 & -0.00160900 \\
\hline $\mathrm{H}$ & 0.79939800 & 7.14467800 & 0.39077500 \\
\hline $\mathrm{H}$ & 2.43121400 & 5.29284900 & 0.54495300 \\
\hline $\mathrm{H}$ & 1.68553200 & 2.92855600 & 0.27016500 \\
\hline $\mathrm{C}$ & 1.05086100 & -1.35923100 & 0.08921100 \\
\hline $\mathrm{C}$ & 2.83699200 & -2.74906500 & 0.13724800 \\
\hline $\mathrm{C}$ & 1.73434300 & -3.49129800 & 0.41390700 \\
\hline $\mathrm{H}$ & 3.87835900 & -3.01940500 & 0.06145400 \\
\hline $\mathrm{H}$ & 1.61646600 & -4.54128800 & 0.63020400 \\
\hline $\mathrm{N}$ & 0.64865300 & -2.62544500 & 0.38076400 \\
\hline $\mathrm{N}$ & 2.40159800 & -1.44575500 & -0.05752800 \\
\hline $\mathrm{Au}$ & -0.06087600 & 0.36501700 & -0.09833700 \\
\hline $\mathrm{C}$ & 3.27997000 & -0.33408700 & -0.36018600 \\
\hline $\mathrm{C}$ & 3.53670900 & -0.02586800 & -1.71108400 \\
\hline $\mathrm{C}$ & 3.85840600 & 0.37395000 & 0.71334500 \\
\hline $\mathrm{C}$ & 4.39680000 & 1.04895600 & -1.97033500 \\
\hline $\mathrm{C}$ & 4.71159800 & 1.43759200 & 0.39189500 \\
\hline $\mathrm{C}$ & 4.97702400 & 1.77460700 & -0.93340400 \\
\hline $\mathrm{H}$ & 4.61883500 & 1.31603500 & -2.99915200 \\
\hline $\mathrm{H}$ & 5.18175900 & 2.00300600 & 1.19116000 \\
\hline $\mathrm{H}$ & 5.64606600 & 2.60043300 & -1.15866500 \\
\hline $\mathrm{C}$ & -0.71126200 & -3.05078800 & 0.64361100 \\
\hline $\mathrm{C}$ & -1.48482900 & -3.53111000 & -0.43191200 \\
\hline $\mathrm{C}$ & -1.18002900 & -3.01580900 & 1.97264900 \\
\hline $\mathrm{C}$ & -2.77389300 & -3.99254900 & -0.13716400 \\
\hline $\mathrm{C}$ & -2.47928500 & -3.48513400 & 2.20526200 \\
\hline $\mathrm{C}$ & -3.26616500 & -3.97465300 & 1.16548900 \\
\hline $\mathrm{H}$ & -3.39932400 & -4.37338300 & -0.93895600 \\
\hline $\mathrm{H}$ & -2.87478200 & -3.47783000 & 3.21651900 \\
\hline $\mathrm{H}$ & -4.26545400 & -4.34826600 & 1.37204600 \\
\hline $\mathrm{C}$ & 3.61361000 & 0.01346400 & 2.17634200 \\
\hline $\mathrm{H}$ & 2.87834400 & -0.79663300 & 2.21194600 \\
\hline $\mathrm{C}$ & 4.90309400 & -0.50878400 & 2.84224400 \\
\hline $\mathrm{H}$ & 5.68087500 & 0.26268200 & 2.86848200 \\
\hline $\mathrm{H}$ & 4.70138800 & -0.81420200 & 3.87509400 \\
\hline $\mathrm{H}$ & 5.31086700 & -1.37293900 & 2.30608800 \\
\hline $\mathrm{C}$ & 3.02123500 & 1.19347100 & 2.97066000 \\
\hline $\mathrm{H}$ & 3.71321900 & 2.04217900 & 3.01212700 \\
\hline $\mathrm{H}$ & 2.08262900 & 1.54311600 & 2.52625300 \\
\hline $\mathrm{H}$ & 2.81512600 & 0.88747900 & 4.00256500 \\
\hline $\mathrm{C}$ & -0.34013700 & -2.50429200 & 3.13970700 \\
\hline $\mathrm{C}$ & -0.10150900 & -3.60526800 & 4.19199800 \\
\hline $\mathrm{H}$ & 0.37056200 & -4.48877200 & 3.74822800 \\
\hline $\mathrm{H}$ & 0.55395000 & -3.23360200 & 4.98748300 \\
\hline $\mathrm{H}$ & -1.03883700 & -3.92751700 & 4.65892000 \\
\hline $\mathrm{C}$ & -0.97870500 & -3.56426900 & -1.87081600 \\
\hline $\mathrm{H}$ & 0.04168800 & -3.16846700 & -1.88447300 \\
\hline $\mathrm{C}$ & 2.94111000 & -0.81608300 & -2.87277700 \\
\hline $\mathrm{C}$ & 4.04146700 & -1.53820800 & -3.67643500 \\
\hline $\mathrm{H}$ & 4.72905400 & -0.82545500 & -4.14520400 \\
\hline $\mathrm{H}$ & 4.63513700 & -2.20181300 & -3.03788600 \\
\hline $\mathrm{H}$ & 3.59510300 & -2.14366100 & -4.47319900 \\
\hline $\mathrm{C}$ & -1.82893600 & -2.65774300 & -2.78288900 \\
\hline $\mathrm{H}$ & -1.84800400 & -1.62721300 & -2.41120200 \\
\hline
\end{tabular}

$\begin{array}{lll}-1.41795000 & -2.65053500 & -3.79894600\end{array}$

$\begin{array}{lll}-2.86463600 & -3.01047700 & -2.84517900\end{array}$

$\begin{array}{lll}-0.91658600 & -5.00383400 & -2.41851100\end{array}$

$\begin{array}{lll}-0.50104600 & -5.00584100 & -3.43242100\end{array}$

$\begin{array}{lll}-0.28649200 & -5.64503800 & -1.79217700\end{array}$

$\begin{array}{lll}-1.91137500 & -5.46060900 & -2.46723000\end{array}$

$\begin{array}{lll}-0.97132000 & -1.25142800 & 3.77879900\end{array}$

$\begin{array}{lll}-1.08698200 & -0.44699700 & 3.04300300\end{array}$

$\begin{array}{lll}-1.95911700 & -1.46825400 & 4.20119700\end{array}$

$\begin{array}{lll}-0.33737100 & -0.87940000 & 4.59181300\end{array}$

$\begin{array}{lll}0.64014300 & -2.20775900 & 2.75338300\end{array}$

$\begin{array}{llll}2.07988100 & 0.07571500 & -3.78839900\end{array}$

$\begin{array}{lll}1.63600900 & -0.52394700 & -4.59120100\end{array}$

$\begin{array}{lll}1.26694600 & 0.55058400 & -3.22738500\end{array}$

$\begin{array}{llll}2.67430400 & 0.86801800 & -4.25703700\end{array}$

$\begin{array}{lll}2.28048400 & -1.58569900 & -2.46116000\end{array}$

$\begin{array}{lll}-3.48828500 & 1.48521500 & -0.75315100\end{array}$

$\begin{array}{lll}-4.40932400 & 1.70546900 & -1.79258400\end{array}$

$\begin{array}{lll}-3.60776400 & 0.30618600 & 0.01234300\end{array}$

$\begin{array}{lll}-5.41734000 & 0.78817800 & -2.07052700\end{array}$

$\begin{array}{lll}-4.31024700 & 2.58858800 & -2.41432300\end{array}$

$\begin{array}{lll}-4.62355200 & -0.59969200 & -0.23927100\end{array}$

$\begin{array}{lll}-2.91479500 & 0.12231300 & 0.82659100\end{array}$

$\begin{array}{lll}-5.53647700 & -0.37117300 & -1.28540700\end{array}$

$\begin{array}{lll}-6.09619700 & 0.97728400 & -2.89347600\end{array}$

$\begin{array}{lll}-4.73060400 & -1.49924900 & 0.35626100\end{array}$

$\begin{array}{lll}-6.48163500 & -1.32051000 & -1.45541600\end{array}$

$\begin{array}{lll}-7.46344000 & -1.14510900 & -2.47553700\end{array}$

$\begin{array}{lll}-7.00218900 & -1.11153600 & -3.46989600\end{array}$

$\begin{array}{lll}-8.11749200 & -2.01482000 & -2.40751600\end{array}$

$\begin{array}{lll}-8.04917300 & -0.23330300 & -2.30935100\end{array}$

$\begin{array}{lll}-2.71665000 & 3.98383200 & -0.20706300\end{array}$

$\begin{array}{llll}-1.46697400 & 2.28420400 & -1.43287800\end{array}$

$\begin{array}{llll}-4.77180800 & 4.95183000 & -1.10790100\end{array}$

$\begin{array}{lll}-5.09285100 & 5.26505100 & -2.10347000\end{array}$

$\begin{array}{lll}-5.34263900 & 4.07617400 & -0.79202500\end{array}$

$\begin{array}{lll}-4.95398000 & 5.77115700 & -0.40423500\end{array}$

$\begin{array}{llll}-3.42609300 & 4.94159400 & 1.92882800\end{array}$

$\begin{array}{lll}-4.18190900 & 4.69342800 & 2.67594600\end{array}$

$\begin{array}{lll}-2.44362400 & 5.00359300 & 2.40666600\end{array}$

$\begin{array}{lll}-3.67183200 & 5.90769500 & 1.47419600\end{array}$

$\begin{array}{lll}-3.47886500 & 3.87459300 & 0.97393400\end{array}$

$\begin{array}{llll}-3.36853000 & 4.68002900 & -1.23714600\end{array}$

Imaginary Frequency $=-816.2083$

$\begin{array}{lrrr}\text { Int4g } & & & \\ \mathrm{C} & -3.05144600 & -0.08546700 & -2.25086100 \\ \mathrm{C} & -3.71146100 & -0.37214200 & -1.04537500 \\ \mathrm{C} & -4.61988800 & 0.53169000 & -0.51250500 \\ \mathrm{C} & -4.82963100 & 1.74847400 & -1.18090400 \\ \mathrm{C} & -4.15470900 & 2.03919300 & -2.36859600 \\ \mathrm{C} & -3.25853100 & 1.11709300 & -2.92150800 \\ \mathrm{C} & -2.21250900 & -1.24063500 & -2.61819700 \\ \mathrm{C} & -2.33176200 & -2.25865400 & -1.67809400 \\ \mathrm{H} & -5.18838600 & 0.30055700 & 0.38175500 \\ \mathrm{H} & -5.54179900 & 2.46261700 & -0.77799400 \\ \mathrm{H} & -4.33886300 & 2.98123200 & -2.87657500 \\ \mathrm{H} & -2.74697100 & 1.33288500 & -3.85540200 \\ \mathrm{C} & 1.57681600 & -0.38343400 & -0.77307300 \\ \mathrm{C} & 3.36208000 & 0.94821700 & -0.41732700 \\ \mathrm{C} & 3.68463000 & -0.29512800 & 0.02263900 \\ \mathrm{H} & 3.92454600 & 1.86830000 & -0.43340500 \\ \mathrm{H} & 4.58521500 & -0.68238100 & 0.47217300 \\ \mathrm{~N} & 2.57833400 & -1.10290100 & -0.20345500\end{array}$




\begin{tabular}{|c|c|c|c|}
\hline $\mathrm{N}$ & 2.06440400 & 0.87826000 & -0.90449300 \\
\hline $\mathrm{Au}$ & -0.25542800 & -1.03054400 & -1.36513000 \\
\hline $\mathrm{C}$ & 1.36459100 & 2.00431600 & -1.49300200 \\
\hline $\mathrm{C}$ & 1.52265000 & 2.24110000 & -2.87360600 \\
\hline $\mathrm{C}$ & 0.59405600 & 2.83155500 & -0.65228700 \\
\hline $\mathrm{C}$ & 0.86031300 & 3.35203900 & -3.41042700 \\
\hline $\mathrm{C}$ & -0.04442700 & 3.92710300 & -1.24696700 \\
\hline $\mathrm{C}$ & 0.08502300 & 4.18588500 & -2.60878700 \\
\hline $\mathrm{H}$ & 0.96026800 & 3.57010300 & -4.46951800 \\
\hline $\mathrm{H}$ & -0.64778800 & 4.58833600 & -0.63242000 \\
\hline $\mathrm{H}$ & -0.41473400 & 5.04558500 & -3.04629900 \\
\hline $\mathrm{C}$ & 2.53870800 & -2.51263000 & 0.13500100 \\
\hline $\mathrm{C}$ & 2.91717400 & -3.45050000 & -0.84651800 \\
\hline $\mathrm{C}$ & 2.17656600 & -2.87428200 & 1.44797400 \\
\hline $\mathrm{C}$ & 2.91800300 & -4.79981500 & -0.47182500 \\
\hline $\mathrm{C}$ & 2.19689700 & -4.23873900 & 1.76301500 \\
\hline $\mathrm{C}$ & 2.56474200 & -5.19166600 & 0.81667600 \\
\hline $\mathrm{H}$ & 3.20597100 & -5.55323800 & -1.19863600 \\
\hline $\mathrm{H}$ & 1.92871200 & -4.55686000 & 2.76615500 \\
\hline $\mathrm{H}$ & 2.58206400 & -6.24405400 & 1.08597500 \\
\hline $\mathrm{C}$ & 0.44503500 & 2.58968200 & 0.84663200 \\
\hline $\mathrm{H}$ & 1.00133500 & 1.68309300 & 1.10615900 \\
\hline $\mathrm{C}$ & 1.05217100 & 3.74718600 & 1.66477700 \\
\hline $\mathrm{H}$ & 0.98458000 & 3.53090400 & 2.73685900 \\
\hline $\mathrm{H}$ & 2.10760500 & 3.90444200 & 1.41601700 \\
\hline $\mathrm{H}$ & 0.52356200 & 4.68937100 & 1.48206300 \\
\hline $\mathrm{C}$ & -1.02612800 & 2.34532500 & 1.23563600 \\
\hline $\mathrm{H}$ & -1.64812200 & 3.22401700 & 1.03190700 \\
\hline $\mathrm{H}$ & -1.44882800 & 1.50082000 & 0.68036000 \\
\hline $\mathrm{H}$ & -1.10091200 & 2.12731300 & 2.30729100 \\
\hline $\mathrm{C}$ & 1.78715700 & -1.85621700 & 2.51583400 \\
\hline $\mathrm{C}$ & 2.79343100 & -1.85186600 & 3.68406500 \\
\hline $\mathrm{H}$ & 3.81256300 & -1.64857400 & 3.33661400 \\
\hline $\mathrm{H}$ & 2.52384400 & -1.08065000 & 4.41424700 \\
\hline $\mathrm{H}$ & 2.80701100 & -2.81476000 & 4.20684200 \\
\hline $\mathrm{C}$ & 3.33297600 & -3.05785000 & -2.26119500 \\
\hline $\mathrm{H}$ & 3.24303600 & -1.97108300 & -2.35824400 \\
\hline $\mathrm{C}$ & 2.37866900 & 1.35967500 & -3.77886200 \\
\hline $\mathrm{C}$ & 3.56078200 & 2.14792600 & -4.37778600 \\
\hline $\mathrm{H}$ & 3.21440700 & 2.96060600 & -5.02583500 \\
\hline $\mathrm{H}$ & 4.18585800 & 2.59105000 & -3.59467500 \\
\hline $\mathrm{H}$ & 4.19142300 & 1.48640100 & -4.98226900 \\
\hline $\mathrm{C}$ & 2.40310000 & -3.68355600 & -3.31907900 \\
\hline $\mathrm{H}$ & 1.35854600 & -3.39936000 & -3.15011400 \\
\hline $\mathrm{H}$ & 2.69363400 & -3.34926200 & -4.32175700 \\
\hline $\mathrm{H}$ & 2.45728800 & -4.77781500 & -3.30572000 \\
\hline $\mathrm{C}$ & 4.80721200 & -3.41930100 & -2.53319100 \\
\hline $\mathrm{H}$ & 5.10154500 & -3.08567500 & -3.53459500 \\
\hline $\mathrm{H}$ & 5.47727600 & -2.94575500 & -1.80707000 \\
\hline $\mathrm{H}$ & 4.97196700 & -4.50132600 & -2.48141900 \\
\hline $\mathrm{C}$ & 0.34995000 & -2.09103900 & 3.01966000 \\
\hline $\mathrm{H}$ & -0.36801300 & -2.05132000 & 2.19356400 \\
\hline $\mathrm{H}$ & 0.25035900 & -3.06542600 & 3.51140600 \\
\hline $\mathrm{H}$ & 0.07749700 & -1.32209000 & 3.75197400 \\
\hline $\mathrm{H}$ & 1.80850300 & -0.85837600 & 2.06584800 \\
\hline $\mathrm{C}$ & 1.53496100 & 0.69971000 & -4.88706000 \\
\hline $\mathrm{H}$ & 2.16059600 & 0.03952000 & -5.49833500 \\
\hline $\mathrm{H}$ & 0.72213600 & 0.10147900 & -4.45945900 \\
\hline $\mathrm{H}$ & 1.08989500 & 1.44628200 & -5.55435600 \\
\hline $\mathrm{H}$ & 2.80122800 & 0.55109500 & -3.17375800 \\
\hline $\mathrm{C}$ & -1.87998500 & -3.65030800 & -1.85447700 \\
\hline $\mathrm{C}$ & -1.91616300 & -4.25041000 & -3.12496900 \\
\hline $\mathrm{C}$ & -1.39709600 & -4.41914800 & -0.77153800 \\
\hline $\mathrm{C}$ & -1.49577900 & -5.56418600 & -3.32346300 \\
\hline $\mathrm{H}$ & -2.31351600 & -3.69609800 & -3.97055200 \\
\hline $\mathrm{C}$ & -0.96644600 & -5.72057700 & -0.95987400 \\
\hline $\mathrm{H}$ & -1.35350300 & -3.97538000 & 0.21682100 \\
\hline
\end{tabular}

$\begin{array}{lrrr}\mathrm{C} & -1.01254000 & -6.30977400 & -2.23727600 \\ \mathrm{H} & -1.56048000 & -5.99812700 & -4.31425700 \\ \mathrm{H} & -0.58491700 & -6.31031700 & -0.13312000 \\ \mathrm{O} & -0.57783200 & -7.58857200 & -2.31019300 \\ \mathrm{C} & -0.62966100 & -8.26349600 & -3.56460000 \\ \mathrm{H} & 0.00065700 & -7.76574300 & -4.31180000 \\ \mathrm{H} & -0.24629000 & -9.26681600 & -3.37628800 \\ \mathrm{H} & -1.65865200 & -8.33014800 & -3.93787600 \\ \mathrm{C} & -3.34159700 & -1.78370900 & -0.57176100 \\ \mathrm{H} & -1.77740100 & -1.36980700 & -3.60612300 \\ \mathrm{C} & -3.35991100 & -1.36814000 & 1.81423100 \\ \mathrm{H} & -2.82546200 & -1.75627300 & 2.68304000 \\ \mathrm{H} & -3.33689300 & -0.27342800 & 1.83538400 \\ \mathrm{H} & -4.39726600 & -1.71643200 & 1.83873600 \\ \mathrm{C} & -4.59171600 & -3.86220300 & -0.16492600 \\ \mathrm{H} & -4.28499500 & -4.53846600 & -0.96741000 \\ \mathrm{H} & -3.96480000 & -4.03049300 & 0.71527100 \\ \mathrm{H} & -5.63644300 & -4.05407100 & 0.08958300 \\ \mathrm{O} & -4.55334400 & -2.49368700 & -0.58901600 \\ \mathrm{O} & -2.66784800 & -1.87422800 & 0.66713100\end{array}$

$\begin{array}{lrrr}\text { TS1g' } & & & \\ \mathrm{C} & -3.31846700 & 0.18210200 & 0.25983800 \\ \mathrm{C} & -4.53726100 & -0.42689100 & 0.62924100 \\ \mathrm{C} & -5.65591500 & 0.33896900 & 0.95201600 \\ \mathrm{C} & -5.58415700 & 1.73267600 & 0.89888500 \\ \mathrm{C} & -4.38875500 & 2.35095000 & 0.53197000 \\ \mathrm{C} & -3.26511000 & 1.58346500 & 0.22337100 \\ \mathrm{C} & -2.14413200 & -0.64244800 & -0.08767100 \\ \mathrm{C} & -2.24667400 & -1.89072500 & -0.50348700 \\ \mathrm{H} & -6.57830900 & -0.15615100 & 1.23394800 \\ \mathrm{H} & -6.45828500 & 2.32838000 & 1.14412100 \\ \mathrm{H} & -4.32517100 & 3.43436600 & 0.48970100 \\ \mathrm{H} & -2.33664800 & 2.06925400 & -0.05933000 \\ \mathrm{C} & 1.62898400 & 0.98747800 & 0.45174900 \\ \mathrm{C} & 3.24266800 & 2.56396500 & 0.66018200 \\ \mathrm{C} & 3.80256100 & 1.36553400 & 0.96358500 \\ \mathrm{H} & 3.65664500 & 3.55955500 & 0.63570200 \\ \mathrm{H} & 4.80557900 & 1.10033000 & 1.25817000 \\ \mathrm{~N} & 2.80186200 & 0.41030500 & 0.83032400 \\ \mathrm{~N} & 1.91139000 & 2.31584200 & 0.35083300 \\ \mathrm{Au} & -0.20989000 & 0.11871200 & 0.14200500 \\ \mathrm{C} & 0.95953800 & 3.34779000 & -0.00510700 \\ \mathrm{C} & 0.78355200 & 3.66350400 & -1.36737700 \\ \mathrm{C} & 0.26991700 & 4.00987500 & 1.03098800 \\ \mathrm{C} & -0.13145400 & 4.67764500 & -1.67730200 \\ \mathrm{C} & -0.63059200 & 5.01680500 & 0.66014500 \\ \mathrm{C} & -0.83193400 & 5.34759300 & -0.67760000 \\ \mathrm{H} & -0.29174900 & 4.94996000 & -2.71618400 \\ \mathrm{H} & -1.17515000 & 5.55312700 & 1.43177000 \\ \mathrm{H} & -1.53140600 & 6.13592300 & -0.94178100 \\ \mathrm{C} & 3.00297800 & -1.00242300 & 1.07502600 \\ \mathrm{C} & 3.51163100 & -1.79814700 & 0.02914300 \\ \mathrm{C} & 2.71819100 & -1.51217400 & 2.35758300 \\ \mathrm{C} & 3.71639800 & -3.15697700 & 0.29824400 \\ \mathrm{C} & 2.93883400 & -2.87949800 & 2.56640900 \\ \mathrm{C} & 3.43019300 & -3.69467100 & 1.55030700 \\ \mathrm{H} & 4.11053000 & -3.80075000 & -0.48237700 \\ \mathrm{H} & 2.73247100 & -3.30788500 & 3.54279700 \\ \mathrm{H} & 3.60111200 & -4.75116900 & 1.73794000 \\ \mathrm{C} & 0.47554200 & 3.68538600 & 2.50810600 \\ \mathrm{H} & 1.18267700 & 2.85329800 & 2.58178200 \\ \mathrm{C} & 1.09439300 & 4.87989300 & 3.26171700 \\ \mathrm{H} & 1.27676900 & 4.61581000 & 4.30952300\end{array}$




\begin{tabular}{|c|c|c|c|}
\hline $\mathrm{H}$ & 2.04848800 & 5.18473200 & 2.81737000 \\
\hline $\mathrm{H}$ & 0.42876400 & 5.75023500 & 3.24851500 \\
\hline $\mathrm{C}$ & -0.83384700 & 3.22543200 & 3.17834400 \\
\hline $\mathrm{H}$ & -1.58650700 & 4.02203600 & 3.18287100 \\
\hline $\mathrm{H}$ & -1.26264500 & 2.35963400 & 2.66215600 \\
\hline $\mathrm{H}$ & -0.64511500 & 2.94486500 & 4.22093400 \\
\hline $\mathrm{C}$ & 2.21210100 & -0.64371900 & 3.50582300 \\
\hline $\mathrm{C}$ & 3.21582900 & -0.62062600 & 4.67620700 \\
\hline $\mathrm{H}$ & 4.20370500 & -0.27660100 & 4.35038600 \\
\hline $\mathrm{H}$ & 2.86375000 & 0.05462800 & 5.46422700 \\
\hline $\mathrm{H}$ & 3.33962700 & -1.61418200 & 5.12164700 \\
\hline $\mathrm{C}$ & 3.86332800 & -1.23363900 & -1.34442200 \\
\hline $\mathrm{H}$ & 3.56001600 & -0.18232500 & -1.37016100 \\
\hline $\mathrm{C}$ & 1.54597400 & 2.96393000 & -2.48882900 \\
\hline $\mathrm{C}$ & 2.45827400 & 3.94998200 & -3.24541200 \\
\hline $\mathrm{H}$ & 1.87717500 & 4.73693200 & -3.73899700 \\
\hline $\mathrm{H}$ & 3.17014600 & 4.43677900 & -2.56949800 \\
\hline $\mathrm{H}$ & 3.02950300 & 3.42351600 & -4.01846600 \\
\hline $\mathrm{C}$ & 3.09946100 & -1.94942600 & -2.47422700 \\
\hline $\mathrm{H}$ & 2.01720400 & -1.89563300 & -2.31777100 \\
\hline $\mathrm{H}$ & 3.33241500 & -1.48422000 & -3.43913600 \\
\hline $\mathrm{H}$ & 3.37846100 & -3.00747200 & -2.54158700 \\
\hline $\mathrm{C}$ & 5.38529900 & -1.27978900 & -1.58946700 \\
\hline $\mathrm{H}$ & 5.62995400 & -0.83085400 & -2.55883300 \\
\hline $\mathrm{H}$ & 5.93458700 & -0.73340800 & -0.81456400 \\
\hline $\mathrm{H}$ & 5.75719200 & -2.31066900 & -1.59452600 \\
\hline $\mathrm{C}$ & 0.81482400 & -1.08769700 & 3.97993800 \\
\hline $\mathrm{H}$ & 0.09105600 & -1.04853700 & 3.15807000 \\
\hline $\mathrm{H}$ & 0.83004500 & -2.11070000 & 4.37347700 \\
\hline $\mathrm{H}$ & 0.45936100 & -0.42920100 & 4.78086300 \\
\hline $\mathrm{H}$ & 2.11594500 & 0.38413300 & 3.14244100 \\
\hline $\mathrm{C}$ & 0.58980800 & 2.23600500 & -3.45391000 \\
\hline $\mathrm{H}$ & 1.16177900 & 1.70072900 & -4.22058900 \\
\hline $\mathrm{H}$ & -0.03204700 & 1.50934000 & -2.91903300 \\
\hline $\mathrm{H}$ & -0.07680800 & 2.93769300 & -3.96786700 \\
\hline $\mathrm{H}$ & 2.19090900 & 2.20164400 & -2.04069300 \\
\hline $\mathrm{C}$ & -1.42097400 & -2.96512700 & -1.02889100 \\
\hline $\mathrm{C}$ & -1.07813200 & -2.99364000 & -2.39189500 \\
\hline $\mathrm{C}$ & -0.96073000 & -4.01171100 & -0.20063700 \\
\hline $\mathrm{C}$ & -0.30589900 & -4.02425700 & -2.92340700 \\
\hline $\mathrm{H}$ & -1.42002900 & -2.19218100 & -3.04047300 \\
\hline $\mathrm{C}$ & -0.18616400 & -5.03716800 & -0.71566200 \\
\hline $\mathrm{H}$ & -1.20288600 & -4.00060200 & 0.85825200 \\
\hline $\mathrm{C}$ & 0.14574800 & -5.05601600 & -2.08394600 \\
\hline $\mathrm{H}$ & -0.05783800 & -4.01429100 & -3.97813500 \\
\hline $\mathrm{H}$ & 0.18284400 & -5.83873000 & -0.08436400 \\
\hline $\mathrm{O}$ & 0.90036200 & -6.10131700 & -2.48670400 \\
\hline $\mathrm{C}$ & 1.26629100 & -6.20284800 & -3.86129800 \\
\hline $\mathrm{H}$ & 1.87476700 & -5.34609100 & -4.17535900 \\
\hline $\mathrm{H}$ & 1.85543700 & -7.11672100 & -3.94238800 \\
\hline $\mathrm{H}$ & 0.38107600 & -6.28115700 & -4.50374600 \\
\hline $\mathrm{C}$ & -4.57820300 & -1.92584900 & 0.71252300 \\
\hline $\mathrm{H}$ & -3.94180100 & -2.33224500 & 1.51226800 \\
\hline $\mathrm{C}$ & -4.23372100 & -3.69605700 & -1.06525000 \\
\hline $\mathrm{H}$ & -3.77282100 & -3.78267400 & -2.04685900 \\
\hline $\mathrm{H}$ & -3.85825700 & -4.48174100 & -0.40359200 \\
\hline $\mathrm{H}$ & -5.31828600 & -3.73156000 & -1.15134000 \\
\hline $\mathrm{C}$ & -6.09006100 & -3.49934300 & 1.68073600 \\
\hline $\mathrm{H}$ & -5.53574200 & -4.40179200 & 1.40018100 \\
\hline $\mathrm{H}$ & -5.82319500 & -3.20395200 & 2.70143600 \\
\hline $\mathrm{H}$ & -7.16041200 & -3.70123400 & 1.62887400 \\
\hline $\mathrm{O}$ & -3.89263300 & -2.38076200 & -0.56513400 \\
\hline $\mathrm{O}$ & -5.85086200 & -2.42013700 & 0.76356700 \\
\hline
\end{tabular}

\begin{tabular}{|c|c|c|c|}
\hline \multicolumn{4}{|l|}{ Int $2 g^{\prime}$} \\
\hline $\mathrm{C}$ & -2.98208300 & 0.18492700 & -1.76488700 \\
\hline $\mathrm{C}$ & -3.46314800 & 0.22986800 & -3.12542900 \\
\hline $\mathrm{C}$ & -4.38142000 & 1.23747500 & -3.55772400 \\
\hline $\mathrm{C}$ & -4.79191500 & 2.21319600 & -2.68588300 \\
\hline $\mathrm{C}$ & -4.28993900 & 2.21060400 & -1.36102900 \\
\hline $\mathrm{C}$ & -3.40206400 & 1.24351600 & -0.92453300 \\
\hline $\mathrm{C}$ & -2.12330100 & -0.86715600 & -1.22531800 \\
\hline $\mathrm{C}$ & -2.46585800 & -2.18545100 & -1.34008400 \\
\hline $\mathrm{H}$ & -4.72612400 & 1.22520700 & -4.58642400 \\
\hline $\mathrm{H}$ & -5.48381300 & 2.98575000 & -3.00520300 \\
\hline $\mathrm{H}$ & -4.61448400 & 2.98316600 & -0.66925300 \\
\hline $\mathrm{H}$ & -3.03739000 & 1.26086200 & 0.09674300 \\
\hline $\mathrm{C}$ & 1.39848200 & 0.67778000 & 0.33548700 \\
\hline $\mathrm{C}$ & 3.02011900 & 2.20452700 & 0.77282500 \\
\hline $\mathrm{C}$ & 3.50205300 & 0.98671700 & 1.12491300 \\
\hline $\mathrm{H}$ & 3.46131500 & 3.18769600 & 0.81728600 \\
\hline $\mathrm{H}$ & 4.45107300 & 0.68732700 & 1.54068600 \\
\hline $\mathrm{N}$ & 2.49821000 & 0.06359000 & 0.85516800 \\
\hline $\mathrm{N}$ & 1.73271600 & 1.99941800 & 0.29243500 \\
\hline $\mathrm{Au}$ & -0.36475900 & -0.14775100 & -0.36237200 \\
\hline $\mathrm{C}$ & 0.87523200 & 3.07007500 & -0.16908400 \\
\hline $\mathrm{C}$ & 0.92583200 & 3.43708500 & -1.52856600 \\
\hline $\mathrm{C}$ & 0.05650200 & 3.72700800 & 0.77154000 \\
\hline $\mathrm{C}$ & 0.10449500 & 4.49444200 & -1.93943900 \\
\hline $\mathrm{C}$ & -0.74412800 & 4.77628300 & 0.30264900 \\
\hline $\mathrm{C}$ & -0.72320700 & 5.15673900 & -1.03659100 \\
\hline $\mathrm{H}$ & 0.11898500 & 4.80719000 & -2.97942400 \\
\hline $\mathrm{H}$ & -1.38521200 & 5.30894500 & 0.99919100 \\
\hline $\mathrm{H}$ & -1.34666500 & 5.97939000 & -1.37605100 \\
\hline $\mathrm{C}$ & 2.64215700 & -1.34938700 & 1.13913100 \\
\hline $\mathrm{C}$ & 3.07836600 & -2.20793000 & 0.11109700 \\
\hline $\mathrm{C}$ & 2.40456300 & -1.79187300 & 2.45642700 \\
\hline $\mathrm{C}$ & 3.27070100 & -3.55571900 & 0.43930100 \\
\hline $\mathrm{C}$ & 2.62013400 & -3.14896300 & 2.72655000 \\
\hline $\mathrm{C}$ & 3.04783100 & -4.02361500 & 1.73103200 \\
\hline $\mathrm{H}$ & 3.60620700 & -4.24659500 & -0.32851400 \\
\hline $\mathrm{H}$ & 2.45200500 & -3.52435000 & 3.73183800 \\
\hline $\mathrm{H}$ & 3.20106300 & -5.07381800 & 1.96165500 \\
\hline $\mathrm{C}$ & 0.03017300 & 3.35643400 & 2.25192200 \\
\hline $\mathrm{H}$ & 0.71446800 & 2.51657300 & 2.40674500 \\
\hline $\mathrm{C}$ & 0.52895200 & 4.52184600 & 3.12967500 \\
\hline $\mathrm{H}$ & 0.55633200 & 4.21955200 & 4.18258100 \\
\hline $\mathrm{H}$ & 1.53723800 & 4.83856100 & 2.84079900 \\
\hline $\mathrm{H}$ & -0.12902400 & 5.39466900 & 3.05146600 \\
\hline $\mathrm{C}$ & -1.36804300 & 2.88735300 & 2.69866200 \\
\hline $\mathrm{H}$ & -2.11599700 & 3.68020100 & 2.58318300 \\
\hline $\mathrm{H}$ & -1.69784000 & 2.01902800 & 2.11722300 \\
\hline $\mathrm{H}$ & -1.35137100 & 2.60034600 & 3.75625800 \\
\hline $\mathrm{C}$ & 1.93151800 & -0.86464200 & 3.57287000 \\
\hline $\mathrm{C}$ & 2.95585700 & -0.79051800 & 4.72235500 \\
\hline $\mathrm{H}$ & 3.93787900 & -0.46151200 & 4.36437700 \\
\hline $\mathrm{H}$ & 2.61841400 & -0.08183400 & 5.48705200 \\
\hline $\mathrm{H}$ & 3.08856400 & -1.76396600 & 5.20758900 \\
\hline $\mathrm{C}$ & 3.35970300 & -1.72820300 & -1.30929800 \\
\hline $\mathrm{H}$ & 3.12123900 & -0.66160800 & -1.36397800 \\
\hline $\mathrm{C}$ & 1.82996000 & 2.74121100 & -2.54157200 \\
\hline $\mathrm{C}$ & 2.86691300 & 3.71451500 & -3.13647400 \\
\hline $\mathrm{H}$ & 2.38605900 & 4.52077700 & -3.70182600 \\
\hline $\mathrm{H}$ & 3.47760900 & 4.17675600 & -2.35310800 \\
\hline $\mathrm{H}$ & 3.53831400 & 3.18336200 & -3.82069200 \\
\hline $\mathrm{C}$ & 2.46037400 & -2.44428500 & -2.33579300 \\
\hline $\mathrm{H}$ & 1.40061000 & -2.30837700 & -2.09274200 \\
\hline $\mathrm{H}$ & 2.64170700 & -2.04257800 & -3.33991600 \\
\hline $\mathrm{H}$ & 2.66371800 & -3.52079300 & -2.36770800 \\
\hline $\mathrm{C}$ & 4.85055400 & -1.88564500 & -1.66941400 \\
\hline $\mathrm{H}$ & 5.04275100 & -1.49827400 & -2.67658800 \\
\hline
\end{tabular}




\begin{abstract}
$\begin{array}{llll}5.49202000 & -1.34023600 & -0.96825000\end{array}$
$\begin{array}{lll}5.15848900 & -2.93722300 & -1.65261200\end{array}$

$\begin{array}{lll}0.54094300 & -1.27719300 & 4.09435900\end{array}$

$-0.19820100 \quad-1.28086900 \quad 3.28552700$

$\begin{array}{lll}0.55840900 & -2.27703000 & 4.54307700\end{array}$

$\begin{array}{lll}0.19993300 & -0.57522400 & 4.86404800\end{array}$

$\begin{array}{lll}1.83131500 & 0.14486200 & 3.16243500\end{array}$

$\begin{array}{llll}1.00829900 & 2.05660300 & -3.65144900\end{array}$

$\begin{array}{lll}1.67358800 & 1.52300800 & -4.34048900\end{array}$

$\begin{array}{lll}0.30216200 & 1.33539300 & -3.22415100\end{array}$

$\begin{array}{lll}0.43804400 & 2.78682900 & -4.23741800\end{array}$

$\begin{array}{llll}2.38426000 & 1.95389900 & -2.02152800\end{array}$

$\begin{array}{lll}-1.75864000 & -3.32781400 & -0.72722400\end{array}$

$\begin{array}{lll}-1.66343500 & -4.54342200 & -1.42758500\end{array}$

$-1.19838000 \quad-3.27275600 \quad 0.56548400$

$\begin{array}{lll}-1.00183100 & -5.64767000 & -0.89490600\end{array}$

$-2.10752600 \quad-4.61984300 \quad-2.41561300$

$\begin{array}{lll}-0.54877600 & -4.36719700 & 1.11286600\end{array}$

$\begin{array}{lll}-1.30720100 & -2.36765600 & 1.15333200\end{array}$

$\begin{array}{lll}-0.43259600 & -5.56269600 & 0.38487100\end{array}$

$\begin{array}{lll}-0.93546500 & -6.55984000 & -1.47661400\end{array}$

$\begin{array}{lll}-0.12577100 & -4.32597000 & 2.11014300\end{array}$

$\begin{array}{lll}0.23209400 & -6.57028500 & 1.00519300\end{array}$

$\begin{array}{lll}0.32604400 & -7.83101600 & 0.35177500\end{array}$

$\begin{array}{lll}0.87994200 & -7.75320000 & -0.59238900\end{array}$

$\begin{array}{lll}0.87017600 & -8.48174300 & 1.03761800\end{array}$

$\begin{array}{lll}-0.66676000 & -8.25572000 & 0.15822000\end{array}$

$\begin{array}{lll}-2.96263100 & -0.70785000 & -4.02934900\end{array}$

$\begin{array}{lll}-2.82573100 & -1.74629300 & -6.16259200\end{array}$

$\begin{array}{lll}-3.62901700 & -2.34607600 & -6.59137900\end{array}$

$\begin{array}{lll}-2.33918600 & -1.15473700 & -6.94063300\end{array}$

$\begin{array}{lll}-2.10099200 & -2.38287100 & -5.64877500\end{array}$

$\begin{array}{lll}-3.44351100 & -0.82715700 & -5.23210000\end{array}$

$\begin{array}{lll}-2.13169600 & -1.35285400 & -3.74932500\end{array}$

$\begin{array}{lll}-3.56497900 & -2.55711700 & -2.11500700\end{array}$

$\begin{array}{lll}-4.66035700 & -3.16487600 & -1.41383700\end{array}$

$\begin{array}{lll}-5.10839500 & -2.44961200 & -0.71370000\end{array}$

$\begin{array}{lll}-5.39446700 & -3.44065100 & -2.17365200\end{array}$

$\begin{array}{llll}-4.34031800 & -4.05840400 & -0.86931000\end{array}$
\end{abstract}

$\begin{array}{lrrr}\text { TS2g' } & & & \\ \mathrm{C} & -2.25368900 & 1.44235300 & 1.01013600 \\ \mathrm{C} & -3.33498400 & 2.31121100 & 0.66600900 \\ \mathrm{C} & -4.48577500 & 2.38136800 & 1.46474600 \\ \mathrm{C} & -4.48697800 & 1.70142800 & 2.67331700 \\ \mathrm{C} & -3.36980600 & 0.93266700 & 3.08742400 \\ \mathrm{C} & -2.25327300 & 0.81233100 & 2.28570700 \\ \mathrm{C} & -1.32802500 & 1.24154800 & -0.04403700 \\ \mathrm{C} & -1.91540000 & 1.55554800 & -1.33681100 \\ \mathrm{H} & -5.32398200 & 3.00172600 & 1.16564800 \\ \mathrm{H} & -5.34392500 & 1.78700600 & 3.33529600 \\ \mathrm{H} & -3.40483600 & 0.42511500 & 4.04682800 \\ \mathrm{H} & -1.41136800 & 0.19465900 & 2.58338400 \\ \mathrm{C} & 2.41178100 & -0.39344900 & 0.48427300 \\ \mathrm{C} & 4.59127900 & -0.95960800 & 0.26298000 \\ \mathrm{C} & 4.06078000 & -1.75078400 & 1.23102400 \\ \mathrm{H} & 5.58778200 & -0.90401000 & -0.14600100 \\ \mathrm{H} & 4.49937900 & -2.52690200 & 1.83813100 \\ \mathrm{~N} & 2.72543400 & -1.39015600 & 1.35471200 \\ \mathrm{~N} & 3.56748100 & -0.13403100 & -0.18442900 \\ \mathrm{Au} & 0.56153000 & 0.48612900 & 0.21349200 \\ \mathrm{C} & 3.72434300 & 0.86531900 & -1.22106500 \\ \mathrm{C} & 3.48643200 & 0.49058300 & -2.55905600 \\ \mathrm{C} & 4.13041200 & 2.16248200 & -0.84736500\end{array}$

$\begin{array}{lll}3.66450100 & 1.47449600 & -3.54060100\end{array}$ $\begin{array}{llll}4.29009700 & 3.10398100 & -1.87238700\end{array}$ $\begin{array}{lll}4.06199400 & 2.76596600 & -3.20406000\end{array}$ $\begin{array}{llll}3.49377800 & 1.22223100 & -4.58295200\end{array}$

$\begin{array}{llll}4.60401500 & 4.11344000 & -1.62344800\end{array}$

$\begin{array}{llll}4.20019900 & 3.51049300 & -3.98323100\end{array}$

$\begin{array}{lll}1.79770000 & -1.99922900 & 2.28593900\end{array}$

$\begin{array}{lll}1.05093500 & -3.11551000 & 1.85825300\end{array}$

$\begin{array}{lll}1.69376000 & -1.45515000 & 3.58201800\end{array}$

$\begin{array}{lll}0.16264300 & -3.68216600 & 2.78121800\end{array}$

$\begin{array}{lll}0.78698400 & -2.06272900 & 4.46010600\end{array}$

$\begin{array}{lll}0.02830000 & -3.16238400 & 4.06607400\end{array}$

$\begin{array}{lll}-0.42831100 & -4.54520800 & 2.48932200\end{array}$

$\begin{array}{lll}0.68105600 & -1.67345400 & 5.46844400\end{array}$

$-0.66419500 \quad-3.62152900 \quad 4.76613800$

$\begin{array}{llll}4.39414900 & 2.56825700 & 0.59993000\end{array}$

$\begin{array}{llll}4.24379300 & 1.69081300 & 1.23657300\end{array}$

$\begin{array}{llll}5.84983800 & 3.03331700 & 0.80248100\end{array}$

$\begin{array}{llll}6.02944400 & 3.26690700 & 1.85786500\end{array}$

$\begin{array}{lll}6.56345100 & 2.25910200 & 0.49969200\end{array}$

$\begin{array}{lll}6.07110700 & 3.93526100 & 0.22122100\end{array}$

$\begin{array}{llll}3.39472000 & 3.64350600 & 1.07051700\end{array}$

$\begin{array}{lll}3.49361000 & 4.56642000 & 0.48760000\end{array}$

$\begin{array}{llll}2.36152100 & 3.28979900 & 0.97490900\end{array}$

$\begin{array}{lll}3.57351700 & 3.89433800 & 2.12227300\end{array}$

$\begin{array}{lll}2.52196700 & -0.26284400 & 4.05340800\end{array}$

$3.44495400 \quad-0.64853800 \quad 5.22684100$

$\begin{array}{lll}4.10379000 & -1.48274100 & 4.96155200\end{array}$

$\begin{array}{lll}4.07281600 & 0.20314200 & 5.51173800\end{array}$

$\begin{array}{lll}2.86973400 & -0.94705400 & 6.11035000\end{array}$

$\begin{array}{llll}1.18623700 & -3.72338500 & 0.46522500\end{array}$

$\begin{array}{lll}1.89715400 & -3.11785800 & -0.10573500\end{array}$

$\begin{array}{lll}3.05804300 & -0.91598600 & -2.96715000\end{array}$

$\begin{array}{lll}4.12720800 & -1.59518800 & -3.84645000\end{array}$

$\begin{array}{lll}4.27158500 & -1.05805300 & -4.79054000\end{array}$

$\begin{array}{lll}5.09656700 & -1.64040600 & -3.33762300\end{array}$

$3.82326200-2.61941600-4.08987100$

$\begin{array}{lll}-0.14985500 & -3.69499000 & -0.30222800\end{array}$

$\begin{array}{lll}-0.53474700 & -2.67307100 & -0.39289000\end{array}$

$\begin{array}{lll}-0.01396700 & -4.09807500 & -1.31217800\end{array}$

$\begin{array}{lll}-0.91421900 & -4.30228200 & 0.19557300\end{array}$

$\begin{array}{lll}1.75610000 & -5.15479300 & 0.53512700\end{array}$

$\begin{array}{lll}1.89160300 & -5.55941100 & -0.47414100\end{array}$

$\begin{array}{lll}2.72680300 & -5.17662600 & 1.04293600\end{array}$

$\begin{array}{lll}1.08270900 & -5.82879000 & 1.07644100\end{array}$

$\begin{array}{lll}1.62819800 & 0.93733800 & 4.42243300\end{array}$

$\begin{array}{lll}1.00891700 & 1.24634700 & 3.57263900\end{array}$

$\begin{array}{lll}0.96181900 & 0.70060400 & 5.25980200\end{array}$

$\begin{array}{lll}2.24497800 & 1.79221000 & 4.72224900\end{array}$

$\begin{array}{lll}3.16515500 & 0.05747600 & 3.22756200\end{array}$

$\begin{array}{lll}1.68520600 & -0.90711300 & -3.66837100\end{array}$

$\begin{array}{llll}1.37215700 & -1.93183900 & -3.89824400\end{array}$

$\begin{array}{lll}0.91581700 & -0.44900000 & -3.03667300\end{array}$

$\begin{array}{lll}1.71986700 & -0.35260300 & -4.61297100\end{array}$

$\begin{array}{lll}2.95254500 & -1.52004000 & -2.06059000\end{array}$

$\begin{array}{lll}-2.97452500 & 0.67025600 & -1.93166300\end{array}$

$\begin{array}{lll}-3.46498000 & 0.95591200 & -3.21322500\end{array}$

$\begin{array}{lll}-3.49048800 & -0.45541700 & -1.26226000\end{array}$

$\begin{array}{lll}-4.43729800 & 0.15952100 & -3.81646500\end{array}$

$\begin{array}{llll}-3.06897000 & 1.80308800 & -3.76105800\end{array}$

$-4.46364400-1.24924400-1.84825300$

$\begin{array}{lll}-3.12119300 & -0.72677100 & -0.27989200\end{array}$

$\begin{array}{lll}-4.95126600 & -0.94988400 & -3.13176200\end{array}$

$\begin{array}{llll}-4.78442200 & 0.41139300 & -4.81171200\end{array}$

$\begin{array}{lll}-4.85418100 & -2.12342200 & -1.33749000\end{array}$

$\begin{array}{lll}-5.89666400 & -1.79165400 & -3.61456300\end{array}$

$\begin{array}{llll}-6.42430900 & -1.55513600 & -4.91522900\end{array}$ 


\begin{tabular}{crrr}
$\mathrm{H}$ & -5.64008600 & -1.61002300 & -5.68062500 \\
$\mathrm{H}$ & -7.15454000 & -2.34675000 & -5.08735300 \\
$\mathrm{H}$ & -6.92398900 & -0.58005300 & -4.97232100 \\
$\mathrm{C}$ & -3.07710600 & 3.03718700 & -0.55645200 \\
$\mathrm{C}$ & -3.96340000 & 4.50057700 & -2.19014000 \\
$\mathrm{H}$ & -4.91764300 & 4.63602500 & -2.69928400 \\
$\mathrm{H}$ & -3.70794400 & 5.41098100 & -1.63541700 \\
$\mathrm{H}$ & -3.18205200 & 4.27682300 & -2.92352700 \\
$\mathrm{O}$ & -4.14353400 & 3.39708400 & -1.29175300 \\
$\mathrm{H}$ & -2.26204400 & 3.76098800 & -0.54342700 \\
$\mathrm{O}$ & -1.14171700 & 2.06921500 & -2.34675100 \\
$\mathrm{C}$ & -0.04109400 & 2.92966700 & -2.04503500 \\
$\mathrm{H}$ & 0.88463100 & 2.35629100 & -1.95088600 \\
$\mathrm{H}$ & 0.04474600 & 3.62155800 & -2.88654300 \\
$\mathrm{H}$ & -0.20364500 & 3.48899100 & -1.11591900 \\
$\mathrm{Im}$ & \multicolumn{3}{c}{}
\end{tabular}

$\begin{array}{cccc}\text { Int3g' } & & & \\ \mathrm{C} & -2.96504700 & 0.16378800 & 0.07638500 \\ \mathrm{C} & -4.23063400 & -0.21642100 & -0.44162400 \\ \mathrm{C} & -5.39064200 & 0.40700700 & -0.00099500 \\ \mathrm{C} & -5.27293000 & 1.43380800 & 0.94296800 \\ \mathrm{C} & -4.02133400 & 1.84005700 & 1.44312700 \\ \mathrm{C} & -2.85993300 & 1.21337200 & 1.01519900 \\ \mathrm{C} & -1.91749700 & -0.62890500 & -0.48938800 \\ \mathrm{C} & -2.56993100 & -1.78110200 & -1.20172700 \\ \mathrm{H} & -6.36740400 & 0.11607700 & -0.37400300 \\ \mathrm{H} & -6.16978300 & 1.93830000 & 1.29203000 \\ \mathrm{H} & -3.97277700 & 2.64456500 & 2.17026200 \\ \mathrm{H} & -1.88629600 & 1.50262400 & 1.39906000 \\ \mathrm{C} & 2.09304700 & 0.17321100 & -0.39300200 \\ \mathrm{C} & 4.04257600 & 1.31954900 & -0.31520800 \\ \mathrm{C} & 4.34589200 & 0.01239400 & -0.52656100 \\ \mathrm{H} & 4.67065200 & 2.19085200 & -0.21664800 \\ \mathrm{H} & 5.29292300 & -0.48932500 & -0.64748300 \\ \mathrm{~N} & 3.14113500 & -0.67619600 & -0.57067900 \\ \mathrm{~N} & 2.65970600 & 1.39993200 & -0.23355700 \\ \mathrm{Au} & 0.07219000 & -0.26233200 & -0.41747600 \\ \mathrm{C} & 1.92696200 & 2.62920300 & -0.00650600 \\ \mathrm{C} & 1.51548500 & 3.38310000 & -1.12345700 \\ \mathrm{C} & 1.67404800 & 3.02331200 & 1.32292600 \\ \mathrm{C} & 0.80739900 & 4.56509800 & -0.87156900 \\ \mathrm{C} & 0.96051900 & 4.21370000 & 1.51215800 \\ \mathrm{C} & 0.52896400 & 4.97584300 & 0.42927100 \\ \mathrm{H} & 0.47525000 & 5.17390800 & -1.70710900 \\ \mathrm{H} & 0.74728600 & 4.55141400 & 2.52207900 \\ \mathrm{H} & -0.01780100 & 5.89901400 & 0.60014800 \\ \mathrm{C} & 3.04102300 & -2.11067100 & -0.74850500 \\ \mathrm{C} & 2.88936900 & -2.62166800 & -2.05304100 \\ \mathrm{C} & 3.13794400 & -2.93254800 & 0.39284600 \\ \mathrm{C} & 2.83222200 & -4.01465700 & -2.19118200 \\ \mathrm{C} & 3.08175000 & -4.31757900 & 0.19127800 \\ \mathrm{H} & 0.32051700 & 1.02419200 & 2.72213700 \\ \mathrm{C} & 2.93001400 & -4.85482100 & -1.08492200 \\ \mathrm{H} & 2.72085800 & -4.44605100 & -3.18129700 \\ \mathrm{H} & 3.16874900 & -4.98276100 & 1.04539200 \\ \mathrm{H} & 2.89702800 & -5.93253000 & -1.21950600 \\ \mathrm{H} & 2.14414800 & 2.22078900 & 2.53338000 \\ \mathrm{C} & 2.71419000 & 1.35826500 & 2.17393500 \\ \mathrm{H} & 3.08819900 & 3.04838600 & 3.42817700 \\ \mathrm{C} & 3.45982000 & 2.43516400 & 4.25678800 \\ \mathrm{H} & 3.95203300 & 3.41832000 & 2.86497700 \\ \mathrm{H} & 0.95359400 & 3.91557600 & 3.86135100 \\ \mathrm{H} & 1.66959200 & 3.34229200 \\ \mathrm{H} & & & \\ \mathrm{H} & & & \end{array}$

\begin{tabular}{|c|c|c|}
\hline 1.31205600 & 1.07699900 & 4 \\
\hline 9600 & -2.37665000 & 200 \\
\hline 995800 & -2.70501400 & 500 \\
\hline 51152600 & -2.30970000 & 1.70988600 \\
\hline 4796200 & -2.26959400 & 3.35369600 \\
\hline 37148600 & -3.78703400 & 2.44192000 \\
\hline 81235000 & -1.73317200 & -3.29076300 \\
\hline 2.79580900 & -0.68934400 & -2.96185700 \\
\hline 1.81567400 & 2.97177400 & -2.56203000 \\
\hline 2.69325800 & 4.02057000 & -3.27473300 \\
\hline 2.17736300 & 4.98288600 & -3.36642100 \\
\hline 3.62968800 & 4.19532400 & -2.73339800 \\
\hline 2.94337600 & 3.68067100 & -4.28594200 \\
\hline 1.51685400 & -1.97391700 & -4.09034600 \\
\hline 62825900 & -1.83216800 & 37600 \\
\hline 900 & -1.2 & 1700 \\
\hline 500 & -2.9 & -4 \\
\hline 800 & -1.91 & 100 \\
\hline 000 & -1.2 & $-5 .($ \\
\hline 500 & -1.7 & 5900 \\
\hline 500 & -2.9 & 5600 \\
\hline 200 & -2.86 & 600 \\
\hline 500 & -2.64 & 4200 \\
\hline 2.279 & -3.951 & 0700 \\
\hline 2.32253900 & -2.38480900 & 7200 \\
\hline 9700 & -1.28664200 & 1.75366800 \\
\hline 0.52218100 & 2.69 & 7600 \\
\hline 0.764 & 2.36 & 9300 \\
\hline-0.08059400 & 1.919 & -2.8 \\
\hline-0.09528400 & 3.601 & 17800 \\
\hline 2.38441800 & 2.03651400 & -2.54072400 \\
\hline-2.42356500 & -2.85150800 & -0.09653600 \\
\hline-1.22811800 & -3.59002600 & 18200 \\
\hline-3.39660600 & -3.09873500 & 0.89255700 \\
\hline-1.02280700 & -4.58447800 & 0.90075900 \\
\hline-0.46183200 & -3.40229700 & -0.79660100 \\
\hline-3.20021700 & -4.07975500 & 1.84845300 \\
\hline-4.32542400 & -2.54181900 & 3400 \\
\hline-2.01385500 & -4.83 & 6700 \\
\hline-0.09 & -5.1 & 300 \\
\hline-3.95 & -4.2 & 2.6 \\
\hline-1.92 & -5.7 & 2.8 \\
\hline-0.75997900 & -6.58 & 60900 \\
\hline 0.13 & -5.97 & 3.10279900 \\
\hline-0.92 & -7.25 & 83000 \\
\hline-0.61827500 & -7.16776000 & 1.99576100 \\
\hline-4.04756400 & -1.30749000 & -1.47371400 \\
\hline-6.03027100 & -2.26518000 & -2.40768300 \\
\hline-6.64770400 & -3.15578400 & -2.27637800 \\
\hline-6.65822700 & -1.37313500 & -2.28193400 \\
\hline-5.61346200 & -2.26192300 & -3.42507500 \\
\hline-5.00185400 & -2.34047900 & -1.42645300 \\
\hline-4.02386900 & -0.85766300 & -2.47852800 \\
\hline-1.86214100 & -2.10593100 & -2.37665200 \\
\hline-2.37121700 & -3.21417800 & -3.12010800 \\
\hline-3.33524600 & -2.97603600 & -3.58609300 \\
\hline-1.63729400 & -3.41018200 & -3.90397200 \\
\hline-2.48864300 & -4.10544100 & -2.49373800 \\
\hline
\end{tabular}

$\begin{array}{lrrr}\text { TS3g' } & & & \\ \text { C } & 0.11280200 & 3.70563900 & -0.11734400 \\ \mathrm{C} & -0.53032900 & 4.95061900 & -0.15799900 \\ \mathrm{C} & 0.19562900 & 6.12900700 & 0.00779000 \\ \mathrm{C} & 1.56867300 & 6.04440200 & 0.26062500 \\ \mathrm{C} & 2.20662400 & 4.80157900 & 0.32473300\end{array}$




\begin{tabular}{|c|c|c|c|}
\hline $\mathrm{C}$ & 1.48461100 & 3.62239900 & 0.12424000 \\
\hline $\mathrm{C}$ & -0.82943400 & 2.58946200 & -0.46317600 \\
\hline $\mathrm{C}$ & -2.16361000 & 3.24681500 & -0.56686900 \\
\hline $\mathrm{H}$ & -0.27957500 & 7.10212700 & -0.06835300 \\
\hline $\mathrm{H}$ & 2.14485100 & 6.95441800 & 0.40120300 \\
\hline $\mathrm{H}$ & 3.27428100 & 4.75258600 & 0.51797200 \\
\hline $\mathrm{H}$ & 1.98544200 & 2.65917500 & 0.14733000 \\
\hline $\mathrm{C}$ & 0.41031600 & -1.10990200 & 0.95642800 \\
\hline $\mathrm{C}$ & 1.83408700 & -2.72736400 & 1.65568700 \\
\hline $\mathrm{C}$ & 0.57139900 & -3.19210800 & 1.83359600 \\
\hline $\mathrm{H}$ & 2.79428100 & -3.17510600 & 1.85761900 \\
\hline $\mathrm{H}$ & 0.20295700 & -4.12768400 & 2.22362300 \\
\hline $\mathrm{N}$ & -0.28861800 & -2.19041700 & 1.39987600 \\
\hline $\mathrm{N}$ & 1.71833200 & -1.45180600 & 1.11983700 \\
\hline $\mathrm{Au}$ & -0.28150800 & 0.69047200 & 0.21657800 \\
\hline $\mathrm{C}$ & 2.85266500 & -0.60418800 & 0.81300600 \\
\hline $\mathrm{C}$ & 3.40459100 & -0.65789100 & -0.48281300 \\
\hline $\mathrm{C}$ & 3.37425000 & 0.21306300 & 1.83694300 \\
\hline $\mathrm{C}$ & 4.51446100 & 0.15652100 & -0.74108800 \\
\hline $\mathrm{C}$ & 4.48656300 & 1.00361100 & 1.51979600 \\
\hline $\mathrm{C}$ & 5.05062800 & 0.97867200 & 0.24663900 \\
\hline $\mathrm{H}$ & 4.96779100 & 0.14153300 & -1.72768400 \\
\hline $\mathrm{H}$ & 4.92049300 & 1.64247300 & 2.28341500 \\
\hline $\mathrm{H}$ & 5.91631900 & 1.59674900 & 0.02542000 \\
\hline $\mathrm{C}$ & -1.73069200 & -2.31022000 & 1.44748800 \\
\hline $\mathrm{C}$ & -2.38987200 & -2.92503600 & 0.36381300 \\
\hline $\mathrm{C}$ & -2.40514800 & -1.85629800 & 2.59954100 \\
\hline $\mathrm{C}$ & -3.77942200 & -3.07635200 & 0.46110800 \\
\hline $\mathrm{C}$ & -3.79440600 & -2.03165700 & 2.63861000 \\
\hline $\mathrm{C}$ & -4.47522900 & -2.63801200 & 1.58565600 \\
\hline $\mathrm{H}$ & -4.32101900 & -3.55328500 & -0.35052000 \\
\hline $\mathrm{H}$ & -4.34846700 & -1.69827100 & 3.51093500 \\
\hline $\mathrm{H}$ & -5.55123500 & -2.77774100 & 1.64557800 \\
\hline $\mathrm{C}$ & 2.79570800 & 0.25168100 & 3.24900800 \\
\hline $\mathrm{H}$ & 1.90491900 & -0.38405900 & 3.27306600 \\
\hline $\mathrm{C}$ & 3.79442900 & -0.32045900 & 4.27525100 \\
\hline $\mathrm{H}$ & 3.34840100 & -0.32911400 & 5.27611000 \\
\hline $\mathrm{H}$ & 4.08590900 & -1.34647700 & 4.02433700 \\
\hline $\mathrm{H}$ & 4.70829200 & 0.28224800 & 4.32278200 \\
\hline $\mathrm{C}$ & 2.34679600 & 1.67157100 & 3.64578200 \\
\hline $\mathrm{H}$ & 3.19400200 & 2.36502400 & 3.69377000 \\
\hline $\mathrm{H}$ & 1.62106900 & 2.07633600 & 2.93205800 \\
\hline $\mathrm{H}$ & 1.87979400 & 1.65512500 & 4.63711800 \\
\hline $\mathrm{C}$ & -1.69047900 & -1.21033600 & 3.78318800 \\
\hline $\mathrm{C}$ & -1.84515500 & -2.05667300 & 5.06265400 \\
\hline $\mathrm{H}$ & -1.47226000 & -3.07657300 & 4.91703400 \\
\hline $\mathrm{H}$ & -1.28260700 & -1.60354000 & 5.88652600 \\
\hline $\mathrm{H}$ & -2.89306500 & -2.12629800 & 5.37516300 \\
\hline $\mathrm{C}$ & -1.65838200 & -3.42761400 & -0.87749600 \\
\hline $\mathrm{H}$ & -0.59307800 & -3.20442000 & -0.76217400 \\
\hline $\mathrm{C}$ & 2.85783500 & -1.56357900 & -1.58208400 \\
\hline $\mathrm{C}$ & 3.87923900 & -2.65178100 & -1.96978900 \\
\hline $\mathrm{H}$ & 4.79118800 & -2.21301000 & -2.38978700 \\
\hline $\mathrm{H}$ & 4.16963200 & -3.25665300 & -1.10362200 \\
\hline $\mathrm{H}$ & 3.45352300 & -3.32231400 & -2.72485600 \\
\hline $\mathrm{C}$ & -2.13775300 & -2.69799500 & -2.14766400 \\
\hline $\mathrm{H}$ & -2.01150100 & -1.61373900 & -2.05075700 \\
\hline $\mathrm{H}$ & -1.56226300 & -3.03359300 & -3.01785500 \\
\hline $\mathrm{H}$ & -3.19554200 & -2.89756500 & -2.35329900 \\
\hline $\mathrm{C}$ & -1.79135100 & -4.95577400 & -1.03224600 \\
\hline $\mathrm{H}$ & -2.83405100 & -5.25635800 & -1.18496600 \\
\hline $\mathrm{H}$ & -1.21586600 & -5.30108800 & -1.89843300 \\
\hline $\mathrm{H}$ & -1.41992400 & -5.48282400 & -0.14648000 \\
\hline $\mathrm{C}$ & -2.17058100 & 0.23639600 & 4.01248600 \\
\hline $\mathrm{H}$ & -2.01643000 & 0.85341900 & 3.12010500 \\
\hline $\mathrm{H}$ & -3.23564700 & 0.27254100 & 4.26795200 \\
\hline $\mathrm{H}$ & 161587700 & 0.69172 & 4.84070 \\
\hline
\end{tabular}

$\begin{array}{lrrr}\mathrm{H} & -0.62149200 & -1.16178900 & 3.55338100 \\ \mathrm{C} & 2.41574300 & -0.75451900 & -2.81697000 \\ \mathrm{H} & 1.96798100 & -1.41901400 & -3.56480200 \\ \mathrm{H} & 1.67596200 & 0.00724800 & -2.54483900 \\ \mathrm{H} & 3.26138800 & -0.24487100 & -3.29226300 \\ \mathrm{H} & 1.96845000 & -2.07225700 & -1.19678000 \\ \mathrm{C} & -3.46732800 & 2.64443700 & -0.62991900 \\ \mathrm{C} & -4.58045100 & 3.41376300 & -1.05244800 \\ \mathrm{C} & -3.70539900 & 1.29099200 & -0.27498700 \\ \mathrm{C} & -5.85286600 & 2.87536500 & -1.12800500 \\ \mathrm{H} & -4.44215500 & 4.45169300 & -1.32964700 \\ \mathrm{C} & -4.97218200 & 0.74637200 & -0.33351500 \\ \mathrm{H} & -2.88111700 & 0.67732400 & 0.07225700 \\ \mathrm{C} & -6.06208300 & 1.53018600 & -0.76236700 \\ \mathrm{H} & -6.67605100 & 3.49517200 & -1.46212000 \\ \mathrm{H} & -5.15207400 & -0.28347900 & -0.04591000 \\ \mathrm{O} & -7.25241600 & 0.91032600 & -0.78562900 \\ \mathrm{C} & -8.41702400 & 1.63382100 & -1.19397900 \\ \mathrm{H} & -8.32231800 & 1.98030000 & -2.22917300 \\ \mathrm{H} & -9.24135200 & 0.92465600 & -1.12013700 \\ \mathrm{H} & -8.60360100 & 2.48514600 & -0.53001900 \\ \mathrm{C} & -2.01057100 & 4.77619900 & -0.42629300 \\ \mathrm{C} & -2.99617100 & 6.51563100 & 0.90624700 \\ \mathrm{H} & -3.81983100 & 6.62329400 & 1.61527300 \\ \mathrm{H} & -2.08702800 & 6.93605600 & 1.35184500 \\ \mathrm{H} & -3.23613100 & 7.06934800 & -0.01461400 \\ \mathrm{O} & -2.86919800 & 5.12291900 & 0.65339300 \\ \mathrm{H} & -2.32679700 & 5.30488700 & -1.34071500 \\ \mathrm{O} & -1.06682500 & 2.84045900 & -1.99529000 \\ \mathrm{C} & -1.41484900 & 1.72066700 & -2.81677400 \\ \mathrm{H} & -2.33450300 & 1.23541100 & -2.47279600 \\ \mathrm{H} & -1.56324400 & 2.12115000 & -3.82235800 \\ \mathrm{H} & -0.59131300 & 1.00173500 & -2.80280200 \\ \mathrm{H} & & \end{array}$
Imaginary Frequency $=-126.3017$

$\begin{array}{cccc}\text { Int4g' } & & & \\ \mathrm{C} & -3.16923800 & -0.06031100 & -1.66391400 \\ \mathrm{C} & -3.50385400 & -0.03788200 & -0.30113400 \\ \mathrm{C} & -4.13655900 & 1.07492800 & 0.23814800 \\ \mathrm{C} & -4.41557700 & 2.16477800 & -0.60093400 \\ \mathrm{C} & -4.07362400 & 2.13549000 & -1.95581400 \\ \mathrm{C} & -3.44402400 & 1.01453700 & -2.50632100 \\ \mathrm{C} & -2.54443500 & -1.36321200 & -1.97027400 \\ \mathrm{C} & -2.44756600 & -2.15756600 & -0.81575000 \\ \mathrm{H} & -4.43704800 & 1.10995900 & 1.28030000 \\ \mathrm{H} & -4.91608600 & 3.03766300 & -0.19224400 \\ \mathrm{H} & -4.30526200 & 2.98762100 & -2.58792500 \\ \mathrm{H} & -3.17331800 & 0.98519100 & -3.55739900 \\ \mathrm{C} & 1.66471700 & -0.66903300 & -1.00682700 \\ \mathrm{C} & 3.57207500 & 0.52998300 & -0.95666400 \\ \mathrm{C} & 3.91097500 & -0.78296500 & -0.87051500 \\ \mathrm{H} & 4.17562400 & 1.42372600 & -0.96806900 \\ \mathrm{H} & 4.87046300 & -1.26879000 & -0.78964400 \\ \mathrm{~N} & 2.72770000 & -1.50734500 & -0.90453800 \\ \mathrm{~N} & 2.18768300 & 0.58356200 & -1.03912900 \\ \mathrm{Au} & -0.29263100 & -1.18110000 & -1.10285200 \\ \mathrm{C} & 1.42507000 & 1.81088600 & -1.15469700 \\ \mathrm{C} & 1.12371400 & 2.29366400 & -2.44411500 \\ \mathrm{C} & 1.04724800 & 2.47820900 & 0.02714000 \\ \mathrm{C} & 0.40161300 & 3.49087300 & -2.52301800 \\ \mathrm{C} & 0.32772900 & 3.67104700 & -0.11627500 \\ \mathrm{C} & 0.00644700 & 4.17220900 & -1.37479800 \\ \mathrm{H} & 0.15206900 & 3.89814300 & -3.49836300 \\ \mathrm{H} & 0.02053100 & 4.21662400 & 0.77092000 \\ \mathrm{H} & -0.54840600 & 5.10219700 & -1.46143100\end{array}$




\begin{tabular}{|c|c|c|c|}
\hline 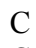 & 2.65643500 & -2.95441700 & -0.85045200 \\
\hline$C$ & 2.73307900 & -3.67239100 & -2.06067500 \\
\hline $\mathrm{C}$ & 2.53859900 & -3.57373200 & 0.40954800 \\
\hline $\mathrm{C}$ & 2.67185300 & -5.06871700 & -1.97721700 \\
\hline $\mathrm{C}$ & 2.48354400 & -4.97288500 & 0.42812600 \\
\hline $\mathrm{C}$ & 2.54628800 & -5.71305100 & -0.74930600 \\
\hline $\mathrm{H}$ & 2.72690800 & -5.65913700 & -2.88689300 \\
\hline $\mathrm{H}$ & 2.39440100 & -5.48817800 & 1.37979100 \\
\hline $\mathrm{H}$ & 2.50270000 & -6.79776300 & -0.71012200 \\
\hline $\mathrm{C}$ & 1.40144700 & 1.96938500 & 1.42181400 \\
\hline $\mathrm{H}$ & 1.93976900 & 1.02186500 & 1.31688900 \\
\hline $\mathrm{C}$ & 2.34211800 & 2.95032000 & 2.15060600 \\
\hline $\mathrm{H}$ & 2.62790200 & 2.54704000 & 3.12857400 \\
\hline $\mathrm{H}$ & 3.25815100 & 3.13095500 & 1.57703800 \\
\hline $\mathrm{H}$ & 1.85934700 & 3.91951200 & 2.31855200 \\
\hline $\mathrm{C}$ & 0.14071000 & 1.68165400 & 2.26030100 \\
\hline $\mathrm{H}$ & -0.45332200 & 2.58951000 & 2.41488600 \\
\hline $\mathrm{H}$ & -0.49747000 & 0.93452300 & 1.77670800 \\
\hline $\mathrm{H}$ & 0.42689100 & 1.30151100 & 3.24795000 \\
\hline $\mathrm{C}$ & 2.49238200 & -2.79367300 & 1.72036100 \\
\hline $\mathrm{C}$ & 3.71936900 & -3.10982900 & 2.59951900 \\
\hline $\mathrm{H}$ & 4.65658300 & -2.90244000 & 2.07105200 \\
\hline $\mathrm{H}$ & 3.70109200 & -2.50130400 & 3.51063300 \\
\hline $\mathrm{H}$ & 3.73543700 & -4.16260600 & 2.90288000 \\
\hline $\mathrm{C}$ & 2.88760300 & -2.99969900 & -3.42203500 \\
\hline $\mathrm{H}$ & 2.92924600 & -1.91642300 & -3.26896200 \\
\hline $\mathrm{C}$ & 1.56186800 & 1.58663900 & -3.72384900 \\
\hline $\mathrm{C}$ & 2.52754800 & 2.46443200 & -4.54495000 \\
\hline $\mathrm{H}$ & 2.04087000 & 3.38361900 & -4.88960300 \\
\hline $\mathrm{H}$ & 3.40552300 & 2.75345000 & -3.95674500 \\
\hline $\mathrm{H}$ & 2.87545800 & 1.92070500 & -5.43040200 \\
\hline $\mathrm{C}$ & 1.67864000 & -3.28067700 & -4.33602500 \\
\hline $\mathrm{H}$ & 0.74562900 & -2.92860100 & -3.88218000 \\
\hline $\mathrm{H}$ & 1.80418300 & -2.76813200 & -5.29677000 \\
\hline $\mathrm{H}$ & 1.57301200 & -4.35160900 & -4.54322200 \\
\hline $\mathrm{C}$ & 4.20655200 & -3.41253300 & -4.10621400 \\
\hline $\mathrm{H}$ & 4.32921000 & -2.87162500 & -5.05121200 \\
\hline $\mathrm{H}$ & 5.07360400 & -3.19229800 & -3.47364400 \\
\hline $\mathrm{H}$ & 4.22433600 & -4.48431300 & -4.33306200 \\
\hline $\mathrm{C}$ & 1.17900700 & -3.04232500 & 2.48762500 \\
\hline $\mathrm{H}$ & 0.30547000 & -2.76116300 & 1.88970800 \\
\hline $\mathrm{H}$ & 1.07162800 & -4.09610200 & 2.76901500 \\
\hline $\mathrm{H}$ & 1.16758000 & -2.45115700 & 3.41098300 \\
\hline $\mathrm{H}$ & 2.52925200 & -1.72462600 & 1.48646700 \\
\hline $\mathrm{C}$ & 0.35412000 & 1.14617800 & -4.57444200 \\
\hline $\mathrm{H}$ & 0.69745900 & 0.61806100 & -5.47141100 \\
\hline $\mathrm{H}$ & -0.30536700 & 0.47297500 & -4.01549800 \\
\hline $\mathrm{H}$ & -0.23908600 & 2.00672000 & -4.90459900 \\
\hline $\mathrm{H}$ & 2.10636500 & 0.67826900 & -3.44618400 \\
\hline $\mathrm{C}$ & -2.20050200 & -3.60702500 & -0.71081100 \\
\hline $\mathrm{C}$ & -2.40960900 & -4.27525600 & 0.50886600 \\
\hline $\mathrm{C}$ & -1.76640000 & -4.38369800 & -1.81216800 \\
\hline $\mathrm{C}$ & -2.23180700 & -5.65137400 & 0.63323800 \\
\hline $\mathrm{H}$ & -2.70751800 & -3.71411500 & 1.38642100 \\
\hline $\mathrm{C}$ & -1.58125700 & -5.74937500 & -1.69762600 \\
\hline $\mathrm{H}$ & -1.56726800 & -3.90043900 & -2.76055300 \\
\hline $\mathrm{C}$ & -1.81740200 & -6.40352100 & -0.47447600 \\
\hline $\mathrm{H}$ & -2.41682700 & -6.12332900 & 1.59101900 \\
\hline $\mathrm{H}$ & -1.25339500 & -6.34274900 & -2.54503600 \\
\hline $\mathrm{O}$ & -1.61400600 & -7.74135100 & -0.47264400 \\
\hline $\mathrm{C}$ & -1.86835700 & -8.47362600 & 0.72252900 \\
\hline $\mathrm{H}$ & -2.91517000 & -8.37482900 & 1.03452000 \\
\hline $\mathrm{H}$ & -1.65833000 & -9.51620600 & 0.48133000 \\
\hline $\mathrm{H}$ & -1.21079100 & -8.14819500 & 1.53832100 \\
\hline $\mathrm{C}$ & -3.10211000 & -1.36600100 & 0.33524800 \\
\hline $\mathrm{C}$ & -2.82837900 & -0.97798700 & 2.69244400 \\
\hline $\mathrm{H}$ & -2.07261600 & -1.16496900 & 3.45798700 \\
\hline
\end{tabular}

